\title{
The Situated Assessment Method (SAM²): Establishing Individual Differences in Habitual Behavior
}

\author{
Léo Dutriaux ${ }^{1}$ \\ Naomi E. Clark ${ }^{1}$ \\ Esther K. Papies ${ }^{1}$ \\ Christoph Scheepers ${ }^{1}$ \\ Lawrence W. Barsalou ${ }^{1}$ \\ ${ }^{1}$ School of Psychology and Neuroscience, University of Glasgow \\ Léo Dutriaux is now at the Center for Mind/Brain Science, University of Trento \\ Naomi Clark is now at the School of Health and Life Sciences, Glasgow Caledonian University
}

Short title: The Situated Assessment Method $\left(\mathrm{SAM}^{2}\right)$

OSF site for materials, data, and analysis scripts: https://osf.io/s34bj/

10 December 2021

Address correspondence to:

Lawrence W. Barsalou

School of Psychology and Neuroscience

School of Psychology

62 Hillhead Street

University of Glasgow

Glasgow G12 8QB UK

+44 (0) 1413303035

lawrence.barsalou@glasgow.ac.uk

http://barsaloulab.org 


\begin{abstract}
From the perspectives of grounded, situated, and embodied cognition, we have developed a new approach for assessing individual differences. Because this approach is grounded in two dimensions of situatedness - situational experience and the Situated Action Cycle - we refer to it as the Situated Assessment Method $\left(\mathrm{SAM}^{2}\right)$. Rather than abstracting over situations during assessment of a construct (as in traditional assessment instruments), SAM $^{2}$ assesses a construct in situations where it occurs, simultaneously measuring factors from the Situated Action Cycle known to influence it. To demonstrate this framework, we developed the SAM ${ }^{2}$ Habitual Behavior Instrument $\left(\mathrm{SAM}^{2} \mathrm{HBI}\right)$. Across three studies with a total of 442 participants, the $\mathrm{SAM}^{2}$ HBI produced a robust and replicable pattern of results at both the group and individual levels. Three trait-level measures of behavior regularity across 80 behaviors, 40 positive behaviors, and 40 negative behaviors exhibited large reliable individual differences. Several sources of evidence demonstrated the construct validity of these measures. At both the group and individual levels, the $\mathrm{SAM}^{2}$ measure of behavior regularity was associated with factors from the Situated Action Cycle known to influence habitual behavior in the literature (consistency, automaticity, immediate reward, long-term reward). Regressions explained approximately $65 \%$ of the variance at the group level and a median of approximately $75 \%$ at the individual level. The $\mathrm{SAM}^{2}$ measure of behavior regularity also exhibited well-established interactions with personality measures for self-control and neuroticism. Cognitive-affective processes from the Situated Action Cycle explained nearly all the variance in these interactions. Finally, a composite measure of habitualness established habitual behaviors at both the group and individual levels. Additionally, a composite measure of reward was strongly related to the composite measure of habitualness, increasing with self-control and decreasing with neuroticism.
\end{abstract}

Keywords: individual differences, psychometrics, habitual behavior, reward, situated cognition, personality 


\section{Introduction}

From the perspectives of grounded, situated, and embodied cognition, the processes that implement human cognition are not abstract, amodal, and decontextualized but instead incorporate situations, embodiment, and action intrinsically into their operation. Decontextualizing cognition by abstracting over situations, embodiment, and action ignores fundamental processes and produces distorted accounts. Research for decades has established the grounded, situated, and embodied perspectives theoretically and empirically $(1-10)$.

Inspired by these perspectives, we have developed a new approach for assessing individual differences. Because this approach is grounded in two dimensions of situatedness-situational experience and the Situated Action Cycle — we refer to it as the Situated Assessment Method ( $\left.\mathrm{SAM}^{2}\right)$. In the remainder of the introduction, we first review properties and limitations of traditional assessment instruments. We then use these properties and limitations to motivate the $\mathrm{SAM}^{2}$ framework. Finally, we present a specific implementation of this framework: the $\mathrm{SAM}^{2} \mathrm{Habitual}$ Behavior Instrument (SAM $\left.{ }^{2} \mathrm{HBI}\right)$.

\section{Traditional assessment instruments}

From the perspective of situated cognition, it is striking how disconnected traditional assessment instruments often are from real-life situations. Typically, assessment instruments use decontextualized items to assess an individual difference of interest. To illustrate, consider an item that assesses conscientiousness in the classic Five Factor Model: "I am someone who does things efficiently" (11-14). In responding to this item, individuals must abstract over situations to establish a general assessment of how much they agree with it. Similarly consider an item from the SelfControl Scale, "I am good at resisting temptation" (15). Again, individuals must abstract over situations to establish a general assessment. Many other assessment instruments similarly ask individuals to respond generally across situations to decontextualized items, including the Perceived Stress Scale (16), the Positive and Negative Affect Schedule (17), the Life Satisfaction Scale (18), the Three-Factor Eating Questionnaire (19), and the Five-Facet Mindfulness Questionnaire (20).

Instrument construction. To develop traditional instruments like these that assess a construct across individuals, an initial pool of decontextualized items related to the construct is developed. To assess neuroticism, for example, items related to worry, moodiness, and reactivity might be sampled. Items can be generated in a bottom up manner from lexical sources such as 
dictionaries (13) or from preliminary qualitative research $(21,22)$. Alternatively, items can be generated in a top-down manner via literature review and expert intuition (12).

To identify a subset of items that coherently measures the target construct, exploratory factor analysis and other methods establish the latent factor structure of the item pool. Related items that only capture the critical construct are then extracted from the pool and assessed again in a preliminary test instrument. Confirmatory factor analysis and other methods verify that all final test items measure the same target construct well.

By aggregating scores across these items into an average or sum, an overall measure for the test is established that orders individuals from high to low on the construct (e.g., high to low neuroticism). Ideally, the aggregate measure should order individuals reliably - it must exhibit satisfactory test reliability. As assessed by Cronbach's alpha and related statistics (23-27), an aggregate measure of a presumably stable trait such as neuroticism should exhibit test reliability of at least .7 to .8 . Intuitively, alpha estimates how highly aggregate scores for individuals on one occasion would correlate with scores from the same test administered to the same individuals again under ideal conditions (e.g., no change in the construct over time, no carry-over effects from the initial test). In other words, alpha estimates how accurately a test captures stable individual differences in the construct assessed.

According to the Spearman-Brown formula, a test's reliability reflects two factors: (a) the coherence between test items (how much they intercorrelate), and (b) the number of test items (2327). In other words, a test's reliability rises as it contains larger numbers of increasingly intercorrelated items. If a test uses 8 items with a coherence of .35 , for example, the resulting alpha is around .80 , establishing that it has satisfactory test reliability.

A satisfactory measure must also demonstrate construct validity - it must adequately measure the construct of interest and only that construct (28). Specifically, the measure should first exhibit content validity, covering all relevant features of the target construct, not excluding any important ones. Additionally the measure should also demonstrate convergent and discriminant validity, capturing the same construct as related measures, while being unrelated to measures for different constructs (29). A measure of neuroticism, for example, should cover all its relevant facets and correlate highly with a measure of emotionality but not with a measure of honesty.

Finally, traditional instruments sometimes assess a related set of constructs, using a single 
instrument to assess multiple subscales. For example, the Five Factor Model uses five subscales to assess five domains of personality: extraversion, agreeableness, openness, conscientiousness, and neuroticism (11). Analogously, the Five Factor Mindfulness Questionnaire assesses five dimensions of mindfulness: awareness, non-reactivity, non-judgmentalness, description, and observation (20). Hierarchical assessment models may contain further subscales. In the Five Factor Inventory, for example, each personality domain is associated with subscales that measure six facets (12). For neuroticism, individual subscales assess the facets of anxiety, angry hostility, depression, selfconsciousness, impulsiveness, and vulnerability. Regardless of how many subscales an instrument includes, the criteria of item coherence, test reliability, and construct validity apply to each.

\section{Limitations of traditional assessment instruments}

A variety of issues bear on the use of traditional assessment instruments: judgment accuracy, situational variance, predictive accuracy, and theoretical understanding. Each is addressed in turn.

Judgment accuracy. What cognitive processes produce responses to decontextualized test items such as, "I am someone who does things efficiently"? It seems unlikely that one can consult a running average in memory for how efficiently one has performed recent tasks, much less over the past year or even a lifetime. So, how does an individual produce a response to this question? How accurate is it? One possibility is that individuals have an intuitive theory about themselves that they consult (30-34). On this account, individuals largely ignore bottom-up empirical data and instead draw inferences from abstract causal theories. Although these theories may reflect actual experience and be somewhat accurate, they may also reflect a variety of beliefs, goals, and biases. Another possibility is that individuals partially sample memories of actual experience but only access memories that are currently available, such that a biased sample results $(35,36)$. To the extent that individuals use intuitive theories and/or the availability heuristic, responses to decontextualized test items are unlikely to be fully accurate (although they can be accurate to some extent; 37,38).

Varying levels of a construct across situations. A tremendous amount of research shows that individuals do not exhibit a constant level of a construct, such as extraversion, across different situations (38-47). Instead, the construct's expression varies widely. Whereas an individual might be extraverted while dining with their family, they might be introverted while dining with coworkers. From the classic theoretical perspective of interactionism $(48,49)$, varying levels of 
extraversion expressed in different situations result from how the individual interacts with situations dynamically over time. Furthermore, expression in a particular situation does simply result from a linear combination of the individual and the situation. Instead, different individuals respond to the same situations differently, such that an individual by situation interaction results. It follows that assessing a construct in different situations for each individual is likely to be useful for both establishing insight into the construct and for characterizing individual differences in it.

Predicting behavior in specific situations. A single fixed measurement of a construct in an individual is unlikely to be accurate at predicting their associated behavior in specific situations. In the attitude literature, much work shows that assessing an attitude in a decontextualized manner often fails to predict associated behavior in specific situations effectively (50-53). If, for example, someone is asked how helpful their behavior is towards others in general, their response may not predict how helpful they are when a stranger asks for directions while hurrying to work. Instead, an accurate prediction is more likely to result when the attitude towards helping others is assessed specifically in this situation. Many additional findings similarly show that general decontextualized measures of personality traits only predict behavior in specific situations weakly $(37,38,43)$

Explaining constructs theoretically. Finally, simply establishing a fixed level of a construct in an individual offers little theoretical insight into it. Often it's assumed that a fixed assessment represents a stable reified disposition within the individual that operates consistently across situations (e.g., neuroticism; 54). Many personality researchers, however, question whether such dispositions literally exist within an individual's cognitive-affective system. Saying, for example, that an individual is neurotic doesn't necessarily mean that an enduring internal disposition for neuroticism exists in an individual's cognitive-affective system, producing related behavior. This attribution makes the same error as concluding that a disposition of briskness exists in external climate conditions when one perceives the weather as "brisk." Instead, briskness, like neuroticism, is a perception that says more about the perceiver than it does about mechanisms in the perceived entity that led to that perception. To mechanistically explain an individual's level of neurotic behavior in specific situations, it is essential to establish the underlying cognitive and affective mechanisms that interact with specific situations to produce varying levels of neuroticism across situations, as well as stable perceived tendencies in behavior $(38-42,44,45,47)$. 


\section{The Situated Assessment Method (SAM²)}

$\mathrm{SAM}^{2}$ is a general assessment framework that can be used to measure diverse constructs, including stress, eating habits, trichotillomania, social connectedness, and sustainable behavior. In this section, we present the general approach. In the next, we present a specific implementation developed to assess habitual behavior.

To establish individual differences in a construct of interest, $\mathrm{SAM}^{2}$ assesses it on two dimensions of situatedness: (a) situational experience, (b) the Situated Action Cycle. The superscript ${ }^{2}$ in $\mathrm{SAM}^{2}$ refers to the fact that $\mathrm{SAM}^{2}$ situates assessment in these two ways. We address each dimension of situatedness in turn.

Capturing situational experience. $\mathrm{SAM}^{2}$ is designed to assess individual differences for a construct in the situations where it occurs. Instead of asking individuals to generalize about the construct across situations without taking them into account explicitly, $\mathrm{SAM}^{2}$ first identifies situations where the construct occurs and then asks individuals to assess the construct in each.

Rather than asking participants to assess how much stress they experience in general, for example, $\mathrm{SAM}^{2}$ asks participants how much stress they experience in specific stress situations (e.g., How much stress do you experience while taking an exam?). Rather than asking participants to assess how much healthy versus unhealthy food they eat in general, $\mathrm{SAM}^{2}$ asks participants how much they eat specific foods in specific eating situations (e.g., How frequently do you eat a salad for dinner?). Rather than asking participants to assess how regularly they perform good versus bad habits in general, $\mathrm{SAM}^{2}$ asks participants how regularly they perform specific habits (e.g., How regularly do you exercise? How regularly do you litter?). A central assumption of $\mathrm{SAM}^{2}$ is that a construct of interest must be assessed in the situations where it occurs.

Our approach to sampling situations is similar to other approaches that sample important life events $(55,56)$, repetitive daily events (57), and situated interaction (58). Like $\mathrm{SAM}^{2}$, all these approaches emphasize the importance of taking an individual's rich situational experience into account. Our approach also resonates strongly with approaches that incorporate the rich structure of everyday experience into psychological research (59-63). Finally, our approach follows longstanding traditions in personality and social psychology that champion the importance of situations in understanding cognition, emotion, and behavior $(38-40,45,64,65)$.

Capturing situational experience with respect to the Situated Action Cycle. To more 
completely understand a construct in the situations where it occurs, it is useful to establish its role in the goal-directed action that transpires in them. Thus, $\mathrm{SAM}^{2}$ doesn't simply measure a construct in relevant situations, it measures additional factors in these situations likely to influence the construct. By doing so, $\mathrm{SAM}^{2}$ establishes both content and convergent validity, comprehensively measuring situational factors related to the target construct of interest.

Rather than simply assessing stress, for example, it's useful to also assess contributing factors that influence stress, such as expectation violation, threat, and coping inefficacy. Rather than simply addressing consumption frequency when assessing eating, it's useful to also assess contributing factors, such as hunger, current emotional state, and health goals. Rather than simply assessing how regularly people perform positive and negative habits, it's useful to also assess contributing factors, such as situational cues, automaticity, and reward.

The Situated Action Cycle provides a powerful theoretical tool for identifying factors that influence a target construct in situations where it occurs (66). Figure 1 illustrates the Situated Action Cycle as an idealized series of discrete linear phases. In actual operation, these phases may overlap in time, occur iteratively, or be omitted. Also, various loops and alternative relations between phases may emerge. The idealized representation in Figure 1 simply illustrates critical phases of the Situated Action Cycle and their approximate relations to each other.

As Figure 1 illustrates, these phases include: the environment, self-relevance, affect, action, and outcomes. In the environment phase, agents experience situations that offer affordances for action and trigger habitual behavioral patterns. In the self-relevance phase, entities and events in the environment implicitly activate relevant goals, values, identities, norms, etc. Once a situation's relevance for the agent is established, it initiates diverse affective states associated with emotion and motivation, often expressed in the body. In turn, emotion and motivation induce diverse actions, ranging from gross bodily movements to eye movements and executive functions. Finally, the actions performed lead to outcomes, such as reward and prediction error. Figure 1 represents the iterative character of the situated action cycle with an arrow that loops back from outcomes to resultant changes in the environment that initiate the next cycle. Figure 1 further represents how each iterative run contributes to conditioning and habit development in long-term memory.

The Situated Action Cycle is hardly a novel idea, with variants playing central roles across disciplines for decades (66). Behavioral conditioning, for example, offers a classic example of the 


\section{The Situated Action Cycle}

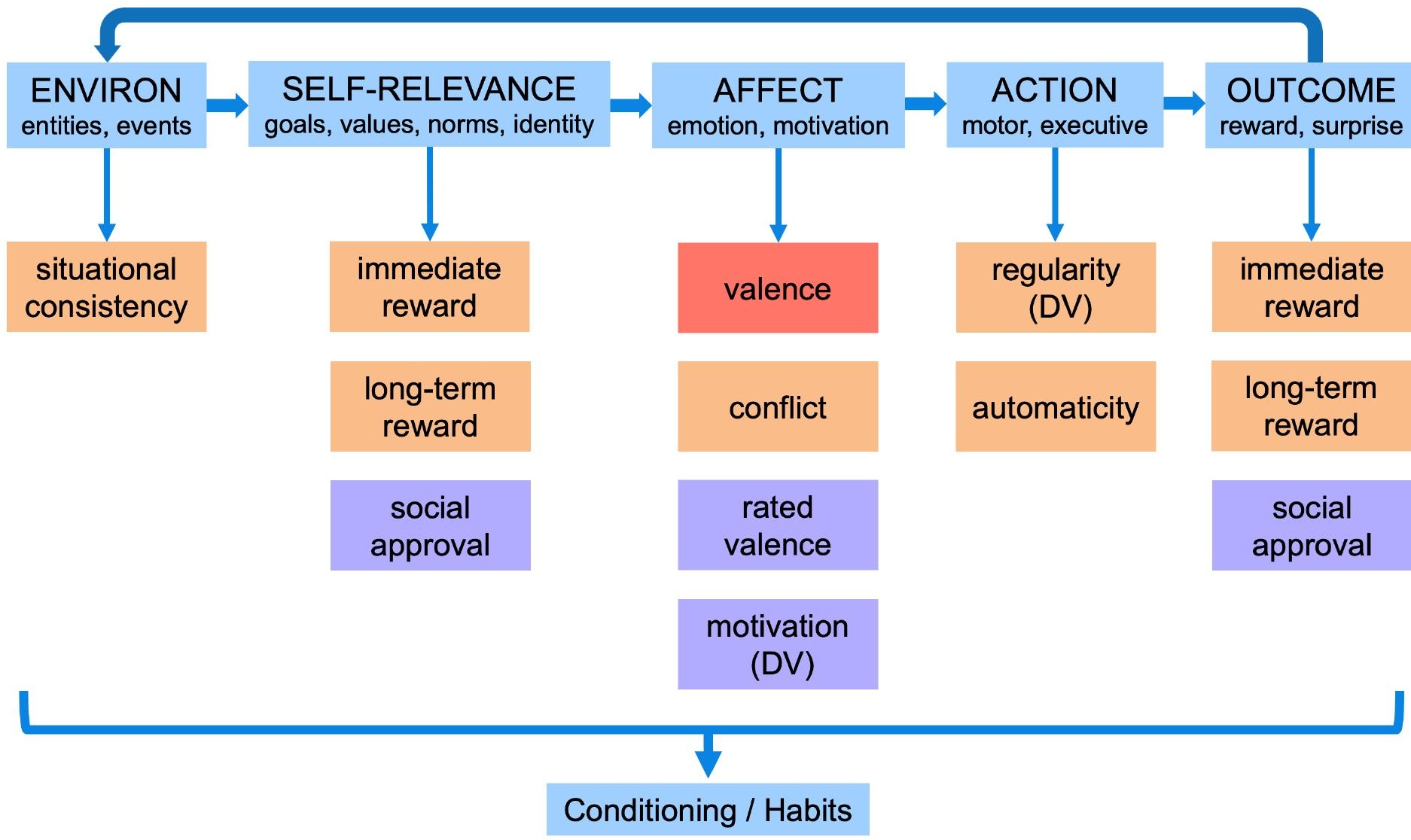

Figure 1. The five phases of the Situated Action Cycle, along with measures sampled from it for the SAM ${ }^{2}$ habits instrument. Valence in red was assigned a priori during the behavior selection process and validated later in Study 2. Measures in orange were assessed across Studies 1-all, Study 2, and Study 3. Measures in purple were only assessed in Study 2 . In later regression analyses, regularity and motivation were dependent variables (DVs), and all other measures were predictors. Measures for the self-relevance and outcome phases of the Situated Action Cycle are the same, given that they can be anticipated initially as self-relevant and then occur later as outcomes. 
Situated Action Cycle in psychology, where environmental cues signal reward outcomes that can be obtained with instrumental actions $(67,68)$. In cognitive science, theories of goal pursuit offer a different take on the Situated Action Cycle, inserting internal cognitive and affective states between the environment, action, and outcomes central to behaviorism. Examples of this expanded approach can be found in computer science approaches to planning and problem solving $(69,70)$, production systems $(71,72)$, reinforcement learning $(73,74)$, and neuroscience $(75-77)$. In discourse analysis, theories of narrative structure propose that conceptual structures similar to the Situated Action Cycle organize people's knowledge of events during event processing and autobiographical memory (78-81). Grounded theories of conceptual processing similarly propose that knowledge becomes organized around situated action $(5,6,66,82,83)$. Because the Situated Action Cycle captures an organized set of processes central to many activities and processes, its ubiquitous role across disciplines is no accident. For this reason, measuring factors across phases of the Situated Action Cycle that influence a target construct is likely to produce comprehensive and informative individual difference measures.

\section{Addressing the limitations of traditional assessment instruments with $\mathrm{SAM}^{2}$}

We next review how the $\mathrm{SAM}^{2}$ framework addresses the four limitations of traditional assessment methods presented earlier.

Judgment accuracy. As we saw earlier, responses to decontextualized items on traditional tests may be inaccurate. Because it is difficult to abstract over all relevant situations accurately, individuals instead fall back on intuitive theories and the availability heuristic. In contrast, SAM ${ }^{2}$ asks participants to evaluate a construct in situations where it occurs (e.g., evaluating stress when performing assignments at work, planning major purchases, resolving disagreement with friends). In the process, each situation serves as a specific retrieval cue to activate relevant memories of the construct in the situation. To the extent that relevant memories become active for each situation, the target construct is evaluated in situations where it occurs. If a relatively representative and comprehensive set of situations is evaluated, the aggregate measure of the construct is likely to be a reasonably accurate summary of the construct in the person's life. Of course, bias and inaccuracy can always occur. Nevertheless, such bias may be less likely and smaller than for items that assess the construct in a completely decontextualized manner (37).

Varying levels of a construct across situations. As we also saw earlier, a second issue for 
traditional assessment instruments is that they only establish a single fixed level of a construct for an individual, ignoring situational variability. In contrast, $\mathrm{SAM}^{2}$ establishes different levels of a construct for an individual across different situations. Because each individual assesses the construct across the same situations, $\mathrm{SAM}^{2}$ further captures individual by situation interactions, namely, how the construct varies differently across situations for different individuals.

Predicting behavior in situations. As we also saw earlier, a third issue for traditional assessment instruments is that they establish a single fixed value of a construct to predict related behavior in different situations, where behavior may vary widely. Because $\mathrm{SAM}^{2}$ establishes different values of the construct in different situations, it makes situation-specific predictions of behavior instead.

Explaining constructs theoretically. Finally, as we saw earlier, traditional instruments offer little theoretical insight into the cognitive-affective mechanisms associated with a construct, simply postulating different stable levels of a decontextualized disposition in different individuals. In contrast, $\mathrm{SAM}^{2}$ establishes the construct within the context of the Situated Action Cycle. By assessing factors from the Cycle that influence the target construct, $\mathrm{SAM}^{2}$ produces a rich theoretical account of it. Specifically, $\mathrm{SAM}^{2}$ establishes how the construct combines with factors in the environment, self-relevance, affect, action, and outcomes to produce situated action in different situations. Rather than simply evaluating the construct in isolation, $\mathrm{SAM}^{2}$ evaluates the construct in the rich context of situated action, thereby resonating with the long tradition of interactionism in psychology $(38-40,45,48)$.

\section{Additional SAM ${ }^{2}$ features}

Item coherence. As we saw earlier, traditional assessment instruments attempt to maximize coherence of the items that assess a latent construct, thereby capturing the construct in a pure and stable manner. In contrast, item coherence is not necessary in $\mathrm{SAM}^{2}$ instruments, or even desirable (where situations constitute the test items). In $\mathrm{SAM}^{2}$ instruments, the construct of interest is assessed using a representative sample of situations that covers the situations where the construct occurs (e.g., How much stress do you experience while taking an exam?). When these situations vary widely, different situations are likely to order individuals differently. Individuals exhibiting high levels of the construct in one situation may exhibiting low levels in another. As a consequence, large individual by situation interactions emerge, producing low item coherence. 
While this may not appear desirable from the traditional assessment perspective, it is desirable from the $\mathrm{SAM}^{2}$ perspective, reflecting the sometimes necessary cost of assessing a target construct comprehensively across a diverse sample of situations.

Importantly, the Spearman-Brown formula indicates how to compensate for low item coherence (23-27). When item coherence is low, assessing a construct with a large number of test items can establish satisfactory test reliability of the aggregate measure. Imagine that coherence is only .05 across situations for a $\mathrm{SAM}^{2}$ instrument. If 80 situations are used, the Spearman-Brown formula estimates that alpha will be .81 , indicating adequate test reliability. Because the aggregate measure is based on so many items, it is reliable, even though coherence is low. Moreover, using such a large number of situations increases the likelihood that the situations assessed do a reasonable job of covering situations where the construct occurs.

Situation-based trait-level measures. Although many constructs exhibit considerable variability across situations, they can also exhibit surprising stability $(38,46,84,85)$. When the varying levels of a construct across situations are aggregated, the resulting 'trait-level' measure tends to be highly stable, typically correlating .7 to .9 with comparable aggregate measures. Individuals, for example, whose aggregate neuroticism is high across multiple assessments over a week tend to also exhibit high neuroticism across multiple assessments the following week. Conversely, individuals whose aggregate neuroticism is low over a week tend to also exhibit low neuroticism the following week. Although one might be tempted to reify this stability into an internal trait mechanism, a more plausible approach is that it reflects an individual-specific configuration of cognitive-affective processes that produces a relatively stable pattern in behavior $(38-40,45,48)$.

By aggregating assessments of a construct for an individual across situations, $\mathrm{SAM}^{2}$ establishes trait-level information about the construct. Importantly, trait-level information in $\mathrm{SAM}^{2}$ is established empirically, statistically aggregating levels across situations. As a consequence, traitlevel measures in $\mathrm{SAM}^{2}$ are likely to differ from traditional decontextualized measures, which again may instead reflect intuitive theories and the availability heuristic. Previous comparisons of these two approaches for establishing trait-level information do indeed demonstrate significant differences $(38,86)$.

Construct validity. As described earlier, a SAM ${ }^{2}$ instrument assesses factors from the Situated Action Cycle known to influence the target construct (as established in the scientific 
literature). If the instrument captures established relations between the construct and these factors, then the instrument exhibits construct validity - it behaves as expected when related influences vary. Imagine, for example, that a $\mathrm{SAM}^{2}$ measure of stress increases with threat across situations while decreasing with coping efficacy. Because this pattern mirrors well-established relationships between stress, threat, and coping in the literature, it demonstrates construct validity for the $\mathrm{SAM}^{2}$ measure of stress.

$\mathrm{SAM}^{2}$ establishes construct validity in this manner at both the group and individual levels. At the group level, $\mathrm{SAM}^{2}$ establishes how robustly a factor influences the construct across both situations and individuals. At the individual level, $\mathrm{SAM}^{2}$ establishes the specific pattern of influence for each individual, together with how much influential factors vary across individuals. Additionally, $\mathrm{SAM}^{2}$ establishes clusters of individuals who show similar patterns of influence.

Finally, data collected with a $\mathrm{SAM}^{2}$ instrument can be used to assess important issues that surround the target construct. For example, it may not be clear whether the construct is associated with a particular factor from the Situated Action Cycle, and if so, how robust this influence is across situations and individuals. Data collected with a $\mathrm{SAM}^{2}$ instrument can address questions like these.

\section{The SAM ${ }^{2}$ Habitual Behavior Instrument (SAM ${ }^{2}$ HBI)}

We developed a $\mathrm{SAM}^{2}$ instrument to assess habitual behavior for several reasons: First, no instrument currently establishes habitual behaviors for an individual across a broad range of human activities. Second, no current instrument evaluates how extensively individuals vary in habitual behavior, nor addresses factors from the Situated Action Cycle that predict these individual differences. Third, a large literature on habits offers considerable guidance in sampling both situations and factors that influence habitual behavior from the Situated Action Cycle. Fourth, no current instrument establishes individual difference measures for positive versus negative habits and relates them to measures of personality, such as self-control and neuroticism. Finally, the SAM ${ }^{2}$ HBI has potential to address important unresolved issues concerning the definition of habit and the relation of reward to habitual behavior.

As the previous section illustrated, developing a $\mathrm{SAM}^{2}$ assessment instrument requires three steps: (a) Identify a representative set of situations where the target construct occurs (to varying degrees, thereby maximizing variance), (b) Identify an appropriate measure of the target construct, (c) Identify scientifically-established factors in the Situated Action Cycle known to influence the 
target construct, maximizing coverage of influential factors. Each step is next addressed in turn for the $\mathrm{SAM}^{2} \mathrm{HBI}$.

Situation selection. To assess situational experience with habitual behaviors, we sampled 80 common behaviors likely to be habitual from 10 domains of human activity (presented in Table 1). Because our initial study assessed habitual behavior in college students, we selected behaviors relevant for this population. All later studies used the same 80 behaviors and continued to assess them in the student population. The 10 behavior domains were established by reviewing the literature on habits and exploring online resources associated with habit change. Our aim was to establish a broad range of common behaviors likely to be habitual across diverse life activities, ranging from food and drink to protecting the environment.

We do not assume that every behavior in Table 1 is a habitual behavior for every individual, but simply assume that these are common habitual behaviors in the population. We offer evidence for this claim later, showing that most of these behaviors were highly habitual for at least some individuals. Within each domain, we manipulated behavior valence, sampling both positive (good) and negative (bad) behaviors. As also discussed later, Study 2 provided strong support for the a priori valence assignments in Table 1, along with insight into the valence distinction: Whereas the positive behaviors were highly associated with long-term reward and social approval, the negative behaviors were not.

Finally, we assume that presenting these behaviors to individuals activates situational memories of performing the respective behavior. As situational memories become active, participants evaluate the target construct being measured. As described earlier, we assume that the level of a construct is likely to vary considerably across situations, and that the memories retrieved for a situation provide reasonably accurate information about the construct's level in each.

Measuring the target construct. For each individual, our goal was to establish the habitualness of each behavior in Table 1. Many measures of habitualness have been proposed in the literature, including the frequency of a behavior, the consistency of the situations that elicit it, the automaticity of the behavior, and the automaticity of the cognitions that precede it. An important related issue is whether reward modulates habitual behavior or not. In general, considerable discussion and disagreement continues to surround these issues (87-101).

Our take on this literature is that the most fundamental property of a habitual behavior is the 
Table 1.

The 80 behaviors assessed for all participants (4 positive and 4 negative behaviors from each of 10 domains).

\section{Positive Habits}

\section{Food and Drink}

1. Eat fruit

2. Eat healthy snacks

3. Eat vegetables

4. Check food labels before making purchases

\section{Exercise}

9. Exercise

10. Walk or bike when possible

11. Participate in sports activities and clubs

12. Take standing and walking breaks when sitting for long periods of time

\section{Affective}

17. Take time to relax

18. Do at least one thing a day that I enjoy and look forward to

19. Express my emotions constructively

20. View challenges with a positive attitude

\section{Social}

25. Maintain contact with family

26. Maintain contact with friends

27. Hold doors open for others

28. Say 'please' and 'thank you'

\section{Technology}

33. Make back-up copies of important documents and files

34. Charge my devices

35. Limit the amount of time each day I spend using technology

36. Restrict my use of technology before sleep

\section{Work and Study}

41. Study for my course(s)

42. Take study breaks

43. Set goals before engaging in a task

44. Pack what I need the night before

\section{Personal Hygiene}
49. Shower every day
50. Cover my mouth when sneezing, coughing or yawning
51. Brush my teeth twice a day
52. Go to sleep and wake up at the same times

\section{Hous ehold}
57. Wash my clothes
58. Put things back after I have finished using them
59. Empty the bins
60. Clean my residence

\section{Finance}
65. Budget
66. Buy from charity and/ or second-hand shops
67. Use shopping lists
68. Shop for groceries

\section{Environment}
73. Turn off lights when leaving a room
74. Recycle
75. Reuse carrier bags
76. Use reusable cups

\section{Negative Habits}

\author{
5. Drink alcohol \\ 6. Eat dessert \\ 7. Eat fast foods \\ 8. Drink soft drinks
}

13. Be sedentary for long periods of time

14. Avoid long walks

15. Reward myself with food and/or drink after exercise

16. Use the lift instead of taking the stairs
21. Use substances to relax
22. Worry
23. Criticise myself
24. Ignore my own needs
29. Use bad language in public
30. Interrupt others
31. Pay little attention to others when they are talking
32. Make myself the centre of conversation

37. Spend a large amount of time on social media

38. Use my phone as a social crutch

(e.g. use my phone when I am alone in social situations)

39. Check my phone multiple times a day

40. Use my phone whilst on the toilet

45. Procrastinate

46. Work whilst watching TV or listening to music

47. Skip lectures

48. Multi-task during work

53. Pick my nose

54. Pick my spots and/or scabs

55. Chew on pencils and/ or pens

56. Bite my nails

61. Allow messes to build up in my work area

62. Ignore stains and spills

63. Leave dishes to wash later

64. Leave clothes lying around

69. Dip into funds I have set aside

70. Spend to make myself feel better

71. Buy brand name products

72. Make impulsive purchases

77. Litter

78. Buy new condition items

79. Leave plug sockets switched on

80. Throw away food 
regularity of performing it when conditions allow. The raw frequency of performing a behavior is not the critical determinant of habitualness - instead it is the regularity of performing the behavior when relevant. From this perspective, attending Christmas Eve mass every year is as habitual as turning off the lights every time one leaves a room, even though the latter occurs much more frequently.

It is certainly possible that behaviors performed regularly are also performed automatically in consistent situations $(91,96)$. Many researchers, however, argue that habitual behaviors are not always performed automatically $(89,94,97,101)$. From the latter perspective, it is an empirical question whether behaviors performed regularly are also performed automatically (and/or consistently). To assess this issue, we included both automaticity and consistency as factors from the Situated Action Cycle that potentially influence regularity (described in the next section).

\section{Assessing factors in the Situated Action Cycle that influence behavior regularity. To}

establish factors from the Situated Action Cycle that influence behavioral regularity, we turned to the large literature on habitual behavior. The red, orange, and purple boxes in Figure 1 illustrate the specific factors in the literature we elected to assess in the studies that follow. We next address factors included in all studies. We then turn to additional factors only assessed in Study 2.

As just described, two important factors associated with habitual behavior are automaticity and consistency. Both were addressed in all studies reported here. On the one hand, consistency contributes to the development, learning, and triggering of habitual behavior $(67,87,96,102,103)$. On the other, as triggering stimuli become frequently and consistently mapped to related responses, automaticity results in both cognition and behavior from repetitive practice $(90,91,99,100,104-106)$. Considerable literature documents the roles of consistency and automaticity in the conditioning process that produces habitual behavior. Based on this literature, we asked participants to assess the consistency and automaticity of each behavior, predicting that both would be strongly related to behavior regularity.

Also of interest was whether individual differences exist in how strongly behavioral regularity is related to consistency and automaticity. Whereas theories that define habitual behavior as consistent and/or automatic assume that these factors are important for all individuals, this prediction has never been tested to our knowledge. From an individual difference perspective, it seems likely that individual differences might well exist in the importance of these two factors, given how much individuals often vary on most measures. 
As noted earlier, a contentious issue in the literature is whether reward modulates habitual behavior or not. To assess this issue, we asked participants in all studies to evaluate both the immediate and the long-term reward of each target behavior. Based on extensive evidence that reward plays a central role in habitual behavior (95), we predicted that both forms of reward would be positively related to behavioral regularity at the group level (also see $88,89,92,97,101$ ). We also predicted, however, that consistency and automaticity would be more strongly related to habitual behavior than reward, reflecting the dominant effects of conditioning that makes habitual behavior somewhat ballistic. Again, however, we expected to observe large individual differences.

Further literature suggests that as a behavior becomes increasingly habitual, the amount of self-regulation accompanying it should decrease (107-109). As a consequence, the amount of conflict experienced with performing the behavior would decrease as well. Whereas conflict should arise as people explicitly decide — and perhaps struggle — with what behavior to perform in a particular situation, conflict should decrease as they settle on a single behavior that becomes increasingly habitual. Thus, we predicted that conflict would decrease at the group level as behavior regularity increased, again though, expecting individual differences.

Assessing relations between personality and habitual behavior. Previous literature shows that self-control is associated with increased performance of positive behaviors and decreased performance of negative behaviors $(97,110-113)$. Conversely, previous literature shows that neuroticism is associated with increased performance of negative behaviors and decreased performance of positive behaviors (114-117). To assess whether the SAM${ }^{2} \mathrm{HBI}$ captures these well-established interactions between personality measures and behavior valence, we included traditional measures of self-control and neuroticism. We also assessed whether factors from the Situated Action Cycle in Figure 1 offer insight into the cognitive and affective processes associated with these personality dispositions.

\section{Methods}

\section{Overview of methods in the individual studies}

Study 1a was an initial exploratory assessment of the SAM ${ }^{2} \mathrm{HBI}$ run in our University of Glasgow laboratory $(n=31)$. Study $1 \mathrm{~b}$ was an exact replication of Study $1 \mathrm{a}$, again run in our Glasgow laboratory $(n=31)$. Study 1c was an exact replication of Studies 1a and 1b combined, run online with participants sampled across the UK from the Prolific platform $(n=66)$. Because these three studies were identical except for the details above, and because they exhibited comparable 
results, they are combined here and referred to as "Study 1-all" $(n=128)$.

Study 2 was an online replication of Study 1-all with a larger UK-wide sample from Prolific $(n=199)$, while assessing three additional factors from the Situated Action Cycle: rated valence, social approval, and motivation (the purple factors in Figure 1).

Study 3 was another replication of Study 1-all using a UK-wide sample from Prolific $(n=115)$. Whereas Study 1-all and Study 2 collected judgments for regularity, consistency, immediate reward, long-term reward, conflict, and automaticity in a fixed blocked order, Study 3 randomized the order of assessing the predictive factors individually, thereby assessing whether the specific format for collecting judgments was important. Study 3 also added an additional metacognitive measure that assessed whether participants were aware of the factors that predicted their regularity judgments.

\section{Common methods across studies}

Participants. All participants were required to be fluent English speakers, UK residents, and students aged 18 to 30 . Online participants (paid £6/hour) were required to have participated in at least 10 Prolific studies and to have achieved at least a 95\% approval rate. No participants were excluded in Studies 1a, 1b, 1c, or 2. In Study 3, two participants were excluded for taking very little time and responding randomly on measures that assessed information in the data (established prior to hypothesis testing).

Design. All participants assessed the same 80 behaviors in Table 1 (40 positive, 40 negative) for the 6 orange factors from the Situated Action Cycle in Figure 1 (behavior regularity consistency, immediate reward, long-term reward, conflict, automaticity). Additionally, all participants received a score for the Brief Self-Control Scale and for the five subscales of the Five Factor Inventory (extraversion, neuroticism, agreeableness, conscientiousness, openness to experience). In a multilevel design, behavior regularity served as the dependent variable, with consistency, immediate reward, long-term reward, conflict, and automaticity predicting regularity at the behavior level, and with self-control and neuroticism predicting regularity at the individual level.

Materials. To cover a broad range of potential habits, we first included behaviors addressed regularly in the habits literature and then turned to internet sites that list habits and help people work with them. Besides attempting to cover as many relevant domains as possible, we manipulated behavior valence, including both positive and negative behaviors (Table 1).

Study 2 assessed the validity of our a priori assignments for valence, with all related results 
reported in supporting information file: S1. Supplemental Material (SM). As shown there, rated valence in Study 2 verified that the positive behaviors in Table 1 were strongly positive and that the negative behaviors were strongly negative, with no overlap. Regressions further found that the valence manipulation reflected $\mathrm{SAM}^{2}$ predictors from the Situated Action Cycle, with positive behaviors being high in long-term reward and social approval, and with negative behaviors being low.

Procedure. All participants performed the study online in an anonymous Qualtrics survey. Participants first received an information sheet about the study and provided informed consent. To ensure that the construct of habit was not salient to participants, the term "habit" was not mentioned at all in Studies 2 and 3 and was mentioned only once in the introduction of Study 1-all.

Participants then evaluated the 80 behaviors in blocks for behavior regularity, consistency, immediate reward, long-term reward, conflict, and automaticity. Table 2 presents the scales used to collect these judgments. As described shortly, the format for collecting these judgments varied across Studies 1-all, 2, and 3. Additionally, Study 2 included judgments for habit motivation, valence, and social approval (described in the SM).

After making these judgments, participants completed the Five Factor Inventory (14) and the Brief Self-Control Scale (15). Finally, participants provided demographic information, were debriefed, and thanked for their participation. Participants typically took about 50 to $60 \mathrm{~min}$ to complete the study, with Studies 2 and 3 taking a little longer than Study 1-all.

\section{Unique methods for individual studies}

Data collection mode. To assess whether our results replicated across different modes of data collection, Studies 1a and 1b were performed in our laboratory, whereas Studies 1c, 2, and 3 were performed online.

Data collection format. In Studies 1a, 1b, and 1c, participants performed three blocks of judgments. In the first block, participants assessed each of the 80 behaviors one at a time for both regularity and situational consistency. In the second block, participants assessed each behavior for both immediate reward and long-term reward. In the third block, participants assessed each behavior for both conflict and automaticity. In each block, the same 80 behaviors were randomly ordered for each participant. The SM presents our reasons for blocking judgments in this manner, together with results that justify these reasons.

In Study 2, the data collection format was the same as the procedure of Study 1-all, with the 
Table 2. The left side presents the scale for each of the six measures common across Studies 1-all, 2, and 3. The right side presents values for inter-rater agreement as measured by intra-class correlations (specifically, the ICC2 measure from the ICC function in the R Psych package). In this context, the intraclass correlation estimates how much different individuals agree in how they order behaviors from high to low on a given measure (interrater agreement). Because the ICC2 form of the intraclass correlation estimates random effects, these values are likely to generalize across other individuals from the same population. Values of the ICC2 across all 80 behaviors are shown on the left; values for the 40 positive behaviors are shown in the middle; values for the 40 negative behavior are shown on the right.

\section{Interrater agreement \\ between individuals \\ when judging behaviors (ICC2)}

\section{Scale Name / Query / Values / Labels}

Study:

\begin{tabular}{|c|c|c|c|c|c|c|c|c|}
\hline \multicolumn{3}{|c|}{ All 80 behaviors } & \multicolumn{3}{|c|}{40 positive } & \multicolumn{3}{|c|}{40 negative } \\
\hline 1-all & 2 & 3 & 1-all & 2 & 3 & 1-all & 2 & 3 \\
\hline .32 & .28 & .28 & .26 & .25 & .25 & .30 & .25 & .27 \\
\hline .18 & .14 & .20 & .16 & .13 & .18 & .16 & .12 & .18 \\
\hline .22 & .19 & .21 & .15 & .12 & .13 & .23 & .18 & .25 \\
\hline .59 & .48 & .53 & .09 & .09 & .09 & .13 & .13 & .13 \\
\hline .16 & .08 & .10 & .14 & .06 & .08 & .12 & .08 & .10 \\
\hline .22 & .17 & .18 & .22 & .16 & .18 & .21 & .17 & .20 \\
\hline
\end{tabular}

\section{Automaticity}

How much do you perform this behaviour automatically with little thought or effort? 
following exceptions. Judgments of behavior motivation were added to block 2, and a new block 4 was added that included rated valence and social approval. Blocks 1 and 3 were the same as in Study 1-all. To streamline the main text, results for behavior motivation, rated valence, and social approval are only presented in the SM. In all cases, they complement the main results reported here and do not raise issues that bear on them.

In Study 3, only one judgment was made on each trial instead of two or three. Because behavior regularity was the dependent variable, it was always assessed in a first block of the 80 randomized behaviors (so as not be influenced by the subsequent blocks for the five predictive factors). These factors (consistency, immediate reward, long-term reward, conflict, automaticity) were then assessed one at a time in five subsequent blocks randomized for each participant, with the 80 behaviors randomized within each. Of interest was whether collecting unblocked judgments with the predictive factors in a random order replicated the pattern of results obtained with the blocked judgment procedure.

The SM provides examples of the screens used to collect judgments in all studies. The SM also provides the rationale for implementing blocked judgments initially in Studies 1-all and 2. As will be seen in the main text, data collection format had no impact on the general pattern of results obtained. The SM presents additional evidence bearing on this issue.

Metacognitive judgments. In Study 3, participants were asked to estimate how much influence each of the $\mathrm{SAM}^{2}$ factors - consistency, immediate reward, long-term reward, conflict, and automaticity — has on them when performing the 80 behaviors in Table 1 (just prior to filling out the Five Factor Inventory and self-control instruments). The SM presents results showing that participants were relatively unaware of the factors associated with their judgments.

\section{Results}

For all results in the main text and SM, data and analysis scripts are publicly available online at OSF (https://osf.io/s34bj/). The SM presents the correlation matrix for the measures common across studies, along with judgment means for the 80 behaviors across $\mathrm{SAM}^{2}$ measures.

\section{Individual differences in behavior regularity}

The SAM ${ }^{2} \mathrm{HBI}$ provides an overall trait-level measure of an individual's behavior regularity. Averaging an individual's regularity judgments provides an aggregate measure of how regularly they perform the 80 behaviors in Table 1 . The median of these individual means in Studies 1-all, 2, and 3 was 54.91, 56.25, and 56.29, respectively, with inter-quartile ranges of 
24.27, 21.60, and 21.46. These values provide a general sense of behavior regularity in the population for these kinds of behaviors, and for how much it varies across individuals. Because the scale value of 50 was labeled, "half the time," these values indicate that participants performed the 80 behaviors in Table 1 over half the time, on average, in situations where possible, with some participants exhibiting more regularity and others exhibiting less.

It is important to note, however, that a given individual performed many behaviors much more regularly than their overall mean, indicating that some behaviors were performed very regularly. To see this, consider the heatmap in Figure 2 that visualizes each participant's regularity judgments in Study 2 (the SM presents analogous heatmaps for Studies 1-all and 3). The number below each column corresponds to the number of the corresponding behavior in Table 1 (with positive behaviors on the left and negative behaviors on the right). When a participant performed a behavior $50 \%$ to $60 \%$ of the time when possible, its cell is relatively whitish in the heat map. As can be seen, however, the cells for many behaviors are bright red, indicating that they were performed $80 \%$ to $100 \%$ of time. Later when we address an individual's most habitual behaviors, we will again see that many behaviors were performed with very high regularity.

The $\mathrm{SAM}^{2} \mathrm{HBI}$ further provides trait-level measures for how regularly individuals perform positive and negative behaviors. Specifically, Figure 3 presents two means for how regularly each individual performs the 40 positive behaviors and the 40 negative behaviors in Table 1 . Across studies, the median of these participant means was around 60 for positive behaviors and around 50 for negative behaviors, indicating that positive behaviors were performed more regularly than negative behaviors. Again, however, the bright red cells on the left and right of Figure 2 illustrate that a given individual performed many positive and negative behaviors $80 \%$ to $100 \%$ of the time.

Interestingly, trait-level measures for the regularity of positive and negative behaviors tended to be negatively correlated. When the mean regularity of performing positive behaviors was correlated with the mean regularity of performing negative behaviors across participants, the correlations were -.22 in Study 1-all, -.03 in Study 2, and -.21 in Study $3(t(126)=-2.54, p=.01$; $t(197)=-.16, p=.71 ; t(113)=-2.34, p=.02)$. As individuals tended to perform positive behaviors more regularly, they did not also tend to perform negative behaviors more regularly. In other words, these two individual difference measures exhibited divergent validity, measuring different trait-level constructs in an individual. Later we will see that personality measures for 
self-control and neuroticism coordinate these divergent measures of positive and negative regularity, and that factors from the Situated Action Cycle explain relations between them.

The SAM ${ }^{2} \mathrm{HBI}$ provides additional individual difference measures for factors from the Situated Action Cycle that influence habitual behavior. Consider the individual means for consistency and automaticity in Figure 3, whose median values were generally around 50 or higher across studies. These relatively high values indicate that the 80 behaviors in Table 1 tended to be performed automatically in consistent situations - hallmarks of habitual behavior.

Figure 3 also presents the individual means for immediate reward and long-term reward across positive and negative behaviors. As can be seen, the median values for immediate reward tended to be greater than 0 , indicating that participants tended to find both positive and negative behaviors immediately rewarding. Perhaps surprisingly, positive behaviors were consistently associated with higher immediate reward than were negative behaviors. As Figure 3 further illustrates, long-term reward behaved more as expected, with a positive median value for positive behaviors but with a negative median value for negative behaviors.

Overall, the pattern of results for regularity, consistency, automaticity, immediate reward, and long-term reward across positive and negative behaviors offers construct validity for the $\mathrm{SAM}^{2} \mathrm{HBI}$. Across the Situated Action, Cycle factors related to habitual behavior reflected well established patterns in the literature.

\section{Individual differences in behavior regularity across situations}

We anticipated that large differences would exist in how regularly individuals perform the 80 behaviors in Table 1. Specifically, we expected that the behaviors one individual performed regularly would differ considerably from the behaviors that another individual performed regularly - an individual by behavior interaction. To assess this prediction, we used the intraclass correlation to assess interrater agreement in how regularly participants performed the 80 behaviors (25). Conceptually, the intraclass correlation in this context estimates the average correlation between all possible pairs of individuals in their regularity judgments across behaviors, establishing the overall similarity in their judgments (i.e., interrater agreement).

The first row of Table 2 (presented earlier) displays the intraclass correlations for behavior regularity across studies, with the lower rows presenting values for the other five measures from the Situated Action Cycle. Because these values were computed using the random effects version 
of the intraclass correlation (the ICC2 measure in 24,25), they generalize beyond the current individuals in each study to other individuals in the larger population.

As the columns for all 80 behaviors in Table 2 illustrate, interrater agreement for behavior regularity was only around .30 across studies, suggesting large individual differences in how regularly individuals perform these behaviors. On the average, the regularity of one individual's behaviors only correlated about .30 with the regularity of another individual's behaviors. As the additional values of the ICC2 illustrate for the 40 positive behaviors and for the 40 negative behaviors, comparable levels of interrater agreement for regularity tended to hold within each subset of behaviors as well. Although this low agreement between individuals could simply reflect noise, we will see later that it is highly systematic. We will also see many further findings that continue to document how much behavior regularity differed between individuals.

Figure 2 offers insight into the relatively low interrater agreement for regularity. As can be seen by comparing different columns with one another, regularity varied widely and systematically across the 80 behaviors. Nevertheless, the pattern of regularity across the 80 behaviors varied widely for different individuals, as can be seen by comparing different rows. It is these differences in patterns of regularity across rows that low interrater reliability in Table 2 is capturing.

In general, differences in the columns and rows of Figure 2 capture classic sources of variance associated with the interactionist approach $(38-40,45,48)$. First, individuals exhibit different trait-level forms of a construct, as captured by overall differences in regularity across rows. Second, a construct takes widely varying forms across situations (behaviors), as captured by overall differences in regularity across columns. Third, a construct exhibits an individual by situation interaction, with the construct's varying levels across situations taking different forms for different individuals (reflected in low interrater agreement).

Finally, agreement in Table 2 for other measures from the Situated Action Cycle varied widely, ranging from $\sim .10$ for conflict to $\sim .50$ for long-term reward, with automaticity ( $\sim .18)$, consistency ( . 18), and immediate reward ( .20) falling in between. In general, these values indicate that the $\mathrm{SAM}^{2} \mathrm{HBI}$ exhibits large individual differences across all its measures. The relatively high agreement for long-term reward across all 80 behaviors ( .50) most likely reflects the salient difference between positive and negative behaviors. When agreement was assessed separately for positive and negative behaviors, interrater reliability for long-term reward dropped 
considerably to .10, indicating much less agreement within each behavior subset.

\section{Test reliability}

As discussed earlier, $\mathrm{SAM}^{2}$ establishes an overall trait-level measure of behavior regularity for each individual across the 80 behaviors in Table 1, along with more specific trait-level measures for positive and negative behaviors (Figure 3). Of interest next is how reliable these trait-level measures are (i.e., how accurately they capture individual differences in behavior regularity). We used Cronbach's alpha to establish test reliability $(23,26$; specifically the ICC3k measure in 24,25).

The top half of Table 3 presents alpha for three SAM${ }^{2}$ measures: (a) an individual's judgments aggregated across all 80 behaviors; (b) an individual's judgments aggregated across the 40 positive behaviors; (c) an individual's judgments aggregated across the 40 negative behaviors. As can be seen in the top row for behavior regularity, these alphas are satisfactory, clustering around the conventionally acceptable level of .70 to .80 . The rows immediately below similarly demonstrate acceptable values for factors from the Situated Action Cycle that influence behavior regularity.

The Spearman-Brown formula offers insight into the values of alpha in Table 3 (23-27). According to Spearman-Brown, alpha increases as a function of two parameters: (a) the coherence between test items (how much they intercorrelate), and (b) the number of test items. Holding the number of test items constant, alpha increases with their coherence. Holding coherence constant, alpha increases with the number of test items. As described earlier, tests typically use around 8 test items with a coherence around .35 to establish a satisfactory alpha of at least .70 to .80 .

Given that the $\mathrm{SAM}^{2}$ aggregate measures used 40 to 80 items, the values of alpha at the top of Table 3 might be expected to be much higher than .80 . The relatively low values of item coherence in the lower half of Table 3 explain the relatively low alphas at the top. The fixed-effects version of the intra-class correlation, ICC3, was used to compute these coherence values, reflecting the assumption that a constant set of behaviors is used to assess regularity across occasions (not different sets in each test; 24,25).

For the overall measure of behavior regularity across all 80 behaviors, inter-behavior coherence was around .04 . These low values indicate that the 80 behaviors differed considerably in how they ordered individual participants from high to low on behavior regularity —individuals high in regularity for one behavior were low for another. In the columns of Figure 2, the widely varying patterns of regularity across individuals for different behaviors visualize the low coherence between 
Table 3. For each $\mathrm{SAM}^{2}$ measure, the top half presents Cronbach's alpha (ICC3k), which estimates the reliability of individual scores across assessment occasions (i.e., test reliability). For each study, values of alpha are shown for scores that were aggregated across all 80 behaviors, across the 40 positive behaviors, or across the 40 negative behaviors (computed with the ICC function in the R Psych package). The bottom half presents inter-behavior consistency (ICC3), which measures how much behaviors agree in their orderings of individual participants from high to low on a measure (i.e., the coherence of test items). For each study, inter-behavior consistency is shown for all 80 behaviors, for the 40 positive behaviors, or for the 40 negative behaviors. As captured in the Spearman-Brown formula, Cronbach's alpha increases with both behavior coherence (ICC2) and the number of behaviors aggregated in the overall measure. Both measures (ICC 3k, ICC 3) estimate fixed effects, assuming that the test instrument contains the same 80 behaviors across test occasions.

Cronbach's alpha (ICC3k)

(test reliability of aggregated participant scores)

\begin{tabular}{|c|c|c|c|c|c|c|c|c|c|c|}
\hline \multirow[b]{2}{*}{ Judgment } & \multirow[b]{2}{*}{ Study: } & \multicolumn{3}{|c|}{ All behaviors } & \multicolumn{3}{|c|}{ Positive behaviors } & \multicolumn{3}{|c|}{ Negative behaviors } \\
\hline & & 1-all & 2 & 3 & 1-all & 2 & 3 & 1-all & 2 & 3 \\
\hline regularity & & .74 & .82 & .78 & .82 & .83 & .86 & .80 & .84 & .83 \\
\hline consistency & & .91 & .91 & .87 & .85 & .85 & .87 & .88 & .89 & .85 \\
\hline immediate reward & & .85 & .88 & .88 & .89 & .90 & .93 & .89 & .89 & .89 \\
\hline long-term reward & & .83 & .87 & .90 & .87 & .91 & .90 & .90 & .93 & .95 \\
\hline conflict & & .95 & .95 & .95 & .94 & .94 & .94 & .93 & .90 & .91 \\
\hline automaticity & & .84 & .89 & .85 & .84 & 89 & (T0 & .85 & .86 & .87 \\
\hline
\end{tabular}

Inter-behavior consistency in ordering participants (ICC3)

\begin{tabular}{|c|c|c|c|c|c|c|c|c|c|c|}
\hline \multirow[b]{2}{*}{ Judgment } & \multirow[b]{2}{*}{ Study: } & \multicolumn{3}{|c|}{ All behaviors } & \multicolumn{3}{|c|}{ Positive behaviors } & \multicolumn{3}{|c|}{ Negative behaviors } \\
\hline & & 1-all & 2 & 3 & 1-all & 2 & 3 & 1-all & 2 & 3 \\
\hline regularity & & .03 & .05 & .04 & .10 & .11 & .13 & .09 & .12 & .11 \\
\hline consistency & & .11 & .12 & .08 & .12 & .13 & .14 & .16 & .16 & .13 \\
\hline immediate reward & & .06 & .08 & .08 & .17 & .19 & .26 & .16 & .17 & .17 \\
\hline long-term reward & & .06 & .08 & .10 & .15 & .20 & .19 & .18 & .24 & .30 \\
\hline conflict & & .20 & .19 & .18 & .27 & .29 & .28 & .26 & .18 & .20 \\
\hline automaticity & & .06 & .09 & .07 & .11 & .17 & .18 & .12 & .13 & .14 \\
\hline
\end{tabular}


behaviors. Most importantly, however, 80 test items is sufficient to produce acceptable alphas near .80 , even when coherence is a low .04 (following Spearman-Brown).

Why might we be seeing such low coherence for the $\mathrm{SAM}^{2} \mathrm{HBI}$ ? According to the Situated Assessment Method, measuring a construct effectively requires assessing it in the situations where it occurs. To ensure that an aggregate measure across situations is not biased toward one kind of situation, the behavior should be assessed in a representative sample. It follows that if situations within a representative sample vary widely, then using them as set of $\mathrm{SAM}^{2}$ test items is likely to exhibit low coherence. To the extent that different situations order individuals differently on the construct from high to low, coherence across a representative set of situations could be low.

One potential source of variance in habitual behaviors is whether they are positive or negative. If so, then computing coherence separately for positive vs. negative behaviors should produce greater coherence. As Table 3 illustrates, coherence for regularity increased from .04 to around .11 for both positive and negative behaviors. As a consequence, corresponding values of alpha in the top-half of Table 3 increased as well.

\section{Construct validity with respect to the Situated Action Cycle: Group level}

If the $\mathrm{SAM}^{2}$ measure of behavior regularity is a valid measure of habitualness, then it should be associated with factors known to influence habitualness in the scientific literature (Figure 1). To the extent that regularity behaves as expected when these factors vary, it demonstrates construct validity. If, for example, the $\mathrm{SAM}^{2}$ measure of behavior regularity increases as consistency and automaticity increase, then it acquires construct validity by virtue of exhibiting these well-established relationships. Similarly, if $\mathrm{SAM}^{2}$ regularity increases as immediate and long-term reward increase, but less so than as consistency and automaticity increase, this further establishes construct validity. Additionally, establishing these relationships contextualizes the target construct of interest—behavior regularity. Rather than being measured and conceptualized in isolation, behavior regularity is instead assessed and understood in the context of factors that influence it in situations where it occurs.

We used multilevel mixed-effect modelling to assess whether group-level data collected with the $\mathrm{SAM}^{2} \mathrm{HBI}$ exhibit this pattern of construct validity. The $\mathrm{SAM}^{2}$ measure of behavior regularity was regressed onto the factors in the Situated Action Cycle common across studies in Figure 1. All models included all main effects, two-way interactions, and three-way interactions, together with random intercepts for participants and behaviors, and relevant random slopes. The SM provides a 
detailed account of the regression pipeline, along with detailed regression results for findings reported here and later. Again, all data and regression scripts can be found online at OSF (https://osf.io/s34bj/).

Figure 4 presents the standardized regression coefficients from these analyses (along with the SEs of these coefficients). By virtue of being standardized, these coefficients offer a measure of effect size (i.e., each coefficient represents the standard-deviation-unit change in regularity associated with a one standard-deviation-unit change in the predictive factor). To demonstrate robustness of the coefficients across studies (and especially for small sample sizes), Figure 4 includes coefficients for the three parts of Study 1-all. The value for each coefficient in Figure 4 was obtained from an analysis where random slopes were included for the respective factor, thereby conservatively assessing the generalizability of the respective fixed effect across participants and behaviors.

As Figure 4 illustrates, a common pattern emerged across studies, demonstrating that the $\mathrm{SAM}^{2}$ HBI produces replicable results. The same pattern of results occurred regardless of sample size, ranging from 31 (Studies 1a and 1b) to 199 (Study 2), lab-based data collection (Studies 1a and 1b) versus online data collection (Studies 1c, 2, and 3), or blocked data collection (Studies 1-all and 2) versus randomized data collection (Study 3).

Most importantly, these results establish construct validity for the $\mathrm{SAM}^{2} \mathrm{HBI}$. Consistent with the habits literature, factors associated with conditioning — consistency and automaticity — were most strongly related to behavior regularity. A behavior became more regular as it was performed more automatically in increasingly consistent situations. Additionally, immediate and long-term reward were also associated with behavior regularity, albeit to a weaker extent than consistency and automaticity. Unexpectedly, conflict was not consistently related to regularity, with most standardized coefficients clustering around 0 . Although valence exhibited a weak positive relation with regularity, its most potent effects emerged through interactions with self-control and neuroticism, addressed later.

Finally, these group-level analyses generally explained $65 \%$ to $70 \%$ of the total variance in behavior regularity (the SM provides detailed results). This explanatory success demonstrates content validity of the SAM ${ }^{2} \mathrm{HBI}$ : Its measures across the Situated Action Cycle are sufficiently thorough and complete to explain the bulk of the variance in habitual behavior. 


\section{Construct validity with respect to the Situated Action Cycle: Individual level}

As we will see both here and in the remaining sections, a diverse set of results at the individual level further demonstrate construct validity for the SAM${ }^{2} \mathrm{HBI}$. We first address whether each individual's judgments of consistency, automaticity, immediate reward, long-term reward, and conflict tended to exhibit predicted relations with habitual behavior observed in the literature. As participants judged each of these factors, did their judgments covary with their judgments of behavior regularity? Notably, participants did not appear to be explicitly aware of how the various predictors covary with behavior regularity, given their general inability to report these relationships accurately (see supporting evidence from an analysis of awareness judgments in the SM). Thus, if these factors from the Situated Action Cycle covary with behavior regularity, they are likely to instead reflect implicit structure across habitual behaviors that the $\mathrm{SAM}^{2} \mathrm{HBI}$ captures.

Each row of the heat map in Figure 5 visualizes the correlations between behavior regularity and the five predictive factors from the Situated Action Cycle for each of the 199 participants in Study 2 (the SM presents analogous heatmaps for Studies 1-all and 3). As a cell becomes redder, the correlation approaches +1 ; as it becomes bluer, it approaches -1 ; as it becomes whiter, it approaches 0. Table 4 summarizes the correlations in Figure 5, presenting the $25^{\text {th }}, 50^{\text {th }}$, and $75^{\text {th }}$ quartiles for each correlation across participants in Studies 1-all, 2, and 3.

As both Figure 5 and Table 4 illustrate, the general pattern for individual prediction conformed to the pattern at the group level. Again, consistency and automaticity were most strongly related to behavior regularity, with median correlations around .75 and .70, respectively. Again, immediate and long-term reward were also related to regularity but to a lesser extent, with median correlations around .45 and .35 , respectively. Again, conflict appeared relatively unrelated to regularity. These results further establish construct validity for the SAM ${ }^{2} \mathrm{HBI}$. Not only does it capture well-established relations between constructs at the group level, it also captures them at the individual level.

We hasten to add, however, that considerable individual differences were nevertheless present. In Figure 5, behavior regularity failed to correlate highly with consistency or automaticity for quite a few participants. The absence of correlations for some participants is even more prevalent for reward, especially long-term reward. Furthermore, major differences in individual prediction vectors differentiate clusters of related participants, as captured by the hierarchical clustering dendrogram on the left. 


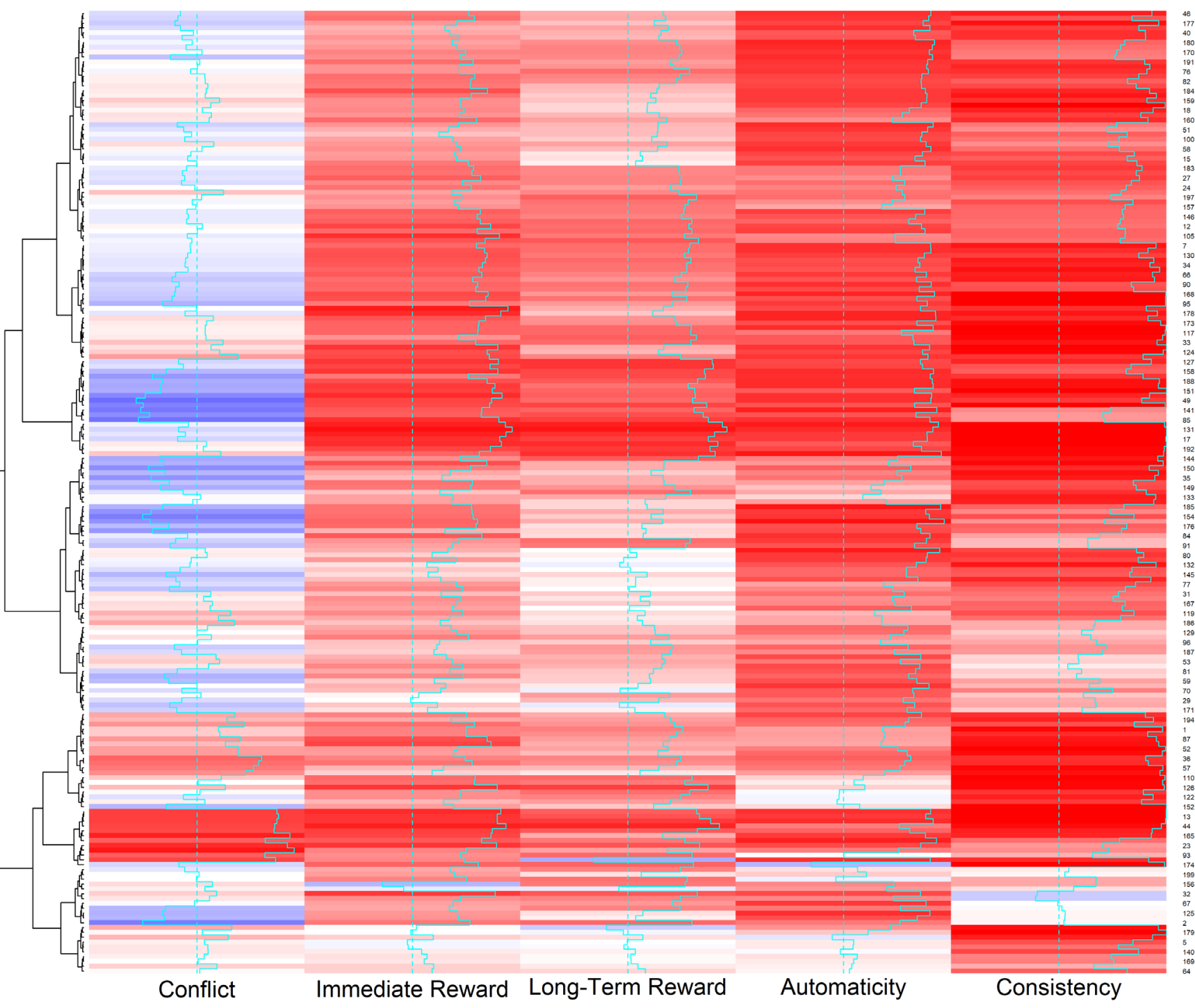

Figure 5. A heatmap that visualizes correlations between behavior regularity and individual factors from the Situated Action Cycle (conflict, immediate reward, long-term reward, automaticity, and consistency). The six correlations for each of the 199 participants in Study 2 appear in a single row. As a cell becomes increasingly red, the correlation was increasingly positive. As a cell becomes increasingly blue, the correlation was increasingly negative. As a cell becomes increasingly white, the correlation increasingly approached 0 . On the left, a hierarchical clustering dendrogram establishes groups of participants having similar prediction vectors (from hierarchical clustering with the Ward D measure). Analogous heatmaps for Studies 1 -all and 3 can be found in the SM. Table 4 summarizes the correlations in all three studies. 
Table 4. Summary of the individual correlations in Figure 5 between behavior regularity and factors from the Situated Action Cycle (Consistency, Immediate Reward, Long-term Reward, Conflict, and Automaticity). For each factor in Study 1-all, 2, or 3, the median correlation across participants is presented, followed by the interquartile range in parentheses. The median is the correlation at the $50^{\text {th }}$ percentile, the first value in parentheses is the correlation at the $25^{\text {th }}$ percentile, and the second value in parentheses is the correlation at the $75^{\text {th }}$ percentile.

\begin{tabular}{|c|c|c|c|c|c|}
\hline Study & Consistency & $\begin{array}{l}\text { Immediate } \\
\text { Reward }\end{array}$ & $\begin{array}{l}\text { Long-Term } \\
\text { Reward }\end{array}$ & Conflict & Automaticity \\
\hline 1-all & $.75(.57-.87)$ & $.49(.38-.58)$ & $.37(.20-.47)$ & $-.08(-.23-.08)$ & $.70(.60-.78)$ \\
\hline 2 & $.72(.53-.89)$ & $.49(.37-.63)$ & $.39(.21-.56)$ & $-.02(-.18-.13)$ & $.66(.48-.77)$ \\
\hline 3 & $.75(.61-.84)$ & $.40(.25-.53)$ & $.31(.10-.49)$ & $-.01(-.16-.12)$ & $.74(.55-.81)$ \\
\hline
\end{tabular}


Content validity in individual regressions. Earlier we saw considerable individual differences in judgments of regularity (Table 2), consistent with a large individual by behavior interaction (Figure 2). Of interest here is whether these large individual differences simply reflected noise or whether they were systemic. If they were systematic, then factors from the Situated Action Cycle in Figure 1 should generally explain high levels of variance in the regularity judgments of individual participants. In other words, the $\mathrm{SAM}^{2} \mathrm{HBI}$ should again illustrate a high level of content validity.

To assess this prediction, a simple linear regression was performed for each participant in Studies 1-all, 2, and 3 on their individually standardized data ( $n=128,199,115$, respectively). Each regression only modeled fixed effects, given that no random effects were logically possible in the individual models. Each regression included behavior regularity as the dependent variable, along with consistency, immediate reward, long-term reward, conflict, and automaticity as predictors. Only main effects were modeled with no interactions. The goal of these simple multiple regressions was to construct a prediction profile of behavior regularity for each individual.

Figure 6 presents the variance explained $\left(R^{2}\right)$ in the individual regressions, with the median $R^{2}$ being $77 \%$ in Study 1-all, 75\% in Study 2, and 72\% in Study 3 (with $R^{2}$ expressed as a percentage). The $R^{2}$ for the same main-effects-only individual model with no random effects or interactions when run at the group level is plotted as the lower dashed purple line. The $R^{2}$ for a group-level model with random intercepts and interactions is plotted as the upper dashed blue line. As can be seen, the median $R^{2}$ for the individual models was higher than the variance explained by both group-level models in every study.

These results for the individual regressions indicate that the relatively low interrater agreement in Table 2 did not reflect noise but instead reflected systematic individual differences. Specifically, high $R^{2}$ values for the individual regressions show that the $\mathrm{SAM}^{2}$ judgments within individuals tended to be highly systematic. As a result, low agreement between individuals for regularity reflected large differences in their systematic assessments of behavior regularity. These results demonstrate the content validity of the $\mathrm{SAM}^{2} \mathrm{HBI}$ at the individual level, explaining even more variance in behavior regularity than at the group level.

\section{Construct validity with respect to self-control and neuroticism}

As described earlier, the literature on habitual behavior has established interactions between personality measures and habit valence. As an individual's self-control increases, they tend to 
perform positive behaviors more and negative ones less (a self-control $\times$ valence interaction). Conversely, as an individual's neuroticism increases, they tend to perform negative behaviors more and positive ones less (a neuroticism $\times$ valence interaction). If the $\mathrm{SAM}^{2}$ measure of behavior regularity is a valid measure of habitualness, it should capture these interactions.

Figures 7 and 8 present these interactions across studies, including the three sub-studies of Study 1-all to demonstrate robustness in small samples. As Figure 7 illustrates, self-control was associated with increasing regularity of positive behaviors and decreasing regularity of negative behaviors across studies. As Figure 8 illustrates, neuroticism was associated with decreasing regularity of positive behaviors and increasing regularity of negative behaviors. The SM presents detailed analyses of these interactions, all remaining robust when random slopes were included for participants and behaviors, demonstrating their generalizability.

These robust findings further establish construct validity for the $\mathrm{SAM}^{2} \mathrm{HBI}$. Consistent with previous literature on relations between personality and habit valence, self-control and neuroticism strongly affected the regularity of positive and negative behaviors in opposite manners.

\section{Using the Situated Action Cycle to understand self-control and neuroticism}

As discussed earlier, viewing self-control and neuroticism as internal trait mechanisms that cause behavior offers limited theoretical understanding of these behavioral dispositions. To understand self-control and neuroticism, it is instead necessary to develop accounts of the underlying cognitive and affective processes as they interact with situations to produce these stable tendencies in behavior $(38-42,44,45,47)$. As will see in these next analyses, the $\mathrm{SAM}^{2}$ framework offers a novel and effective approach for establishing such accounts.

Of interest in these analyses was whether factors from the Situated Action Cycle for habitual behavior in Figure 1 - consistency, automaticity, immediate reward, long-term reward, and conflict — explain variance in self-control and neuroticism. To see this, first consider the selfcontrol $\times$ valence interactions in Figure 7 , which resulted from regressing behavior regularity onto only self-control, valence, and their two-way interaction (including random intercepts and slopes as described in the SM). If factors from the Situated Action Cycle for habitual behavior in Figure 1 underlie self-control, then adding all these factors into the initial regression models should eliminate self-control's interactions with valence. If these factors don't underlie self-control, then the self-control $\times$ valence interactions should remain. 


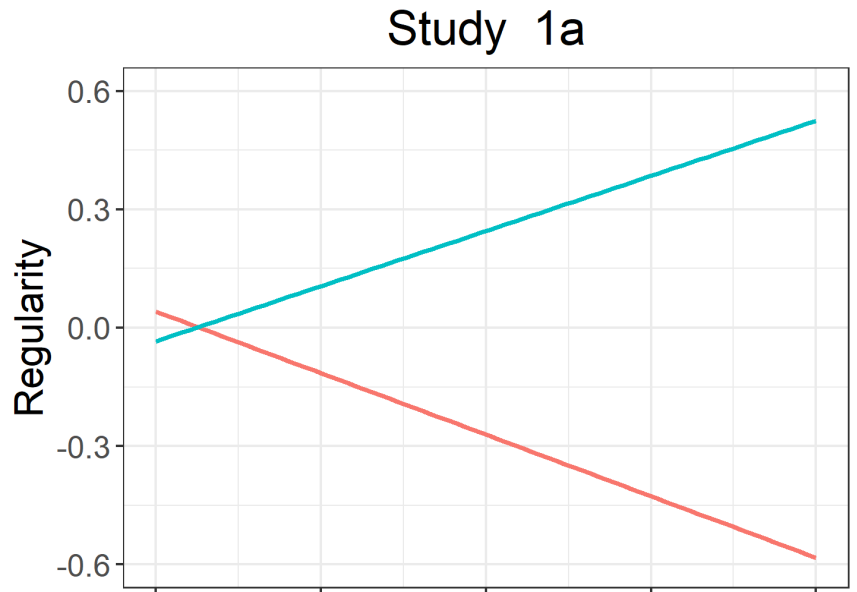

Study $1 b$

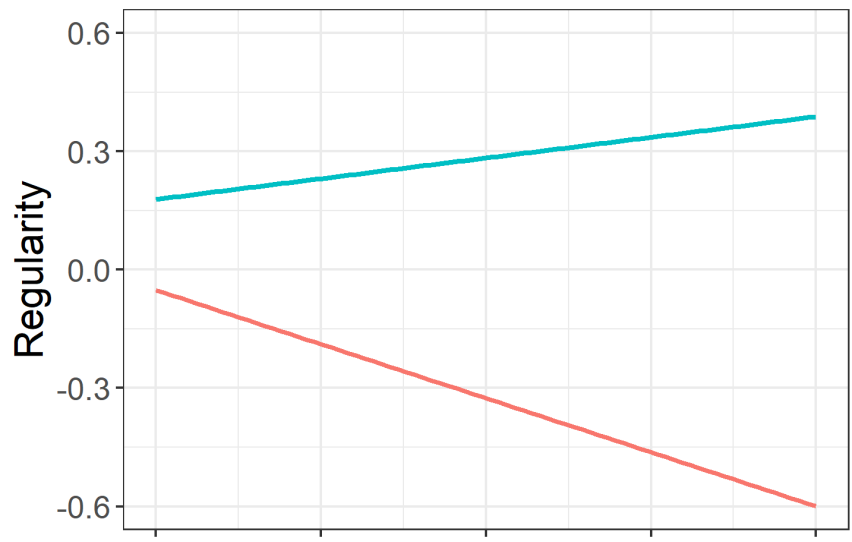

Study 1c

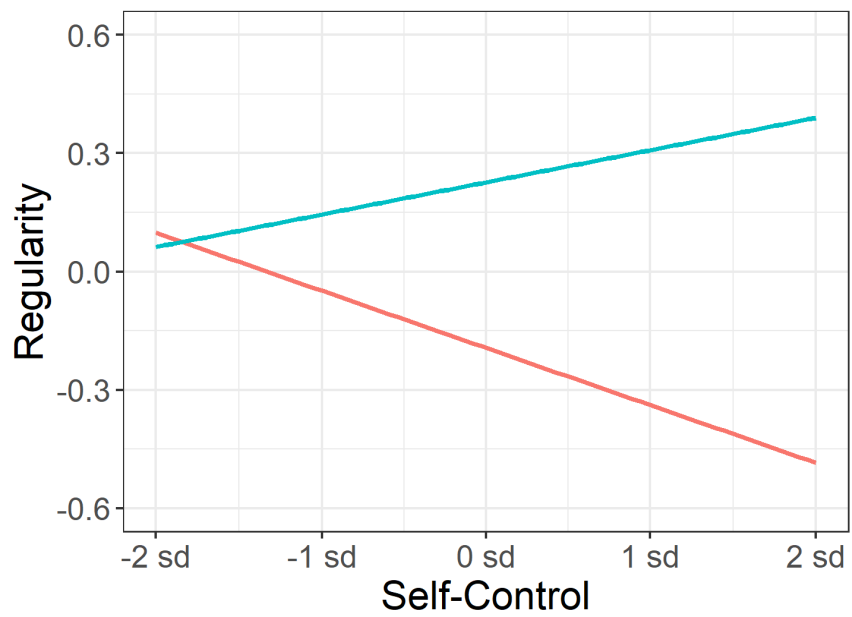

Study 1-all

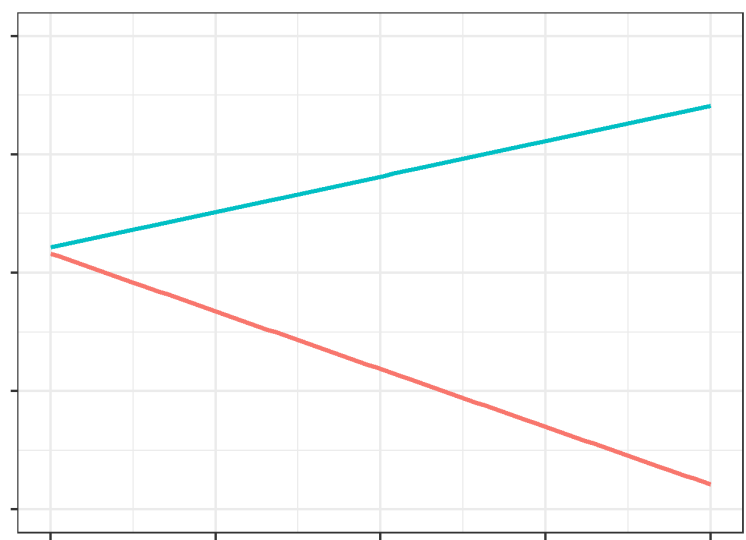

Study 2

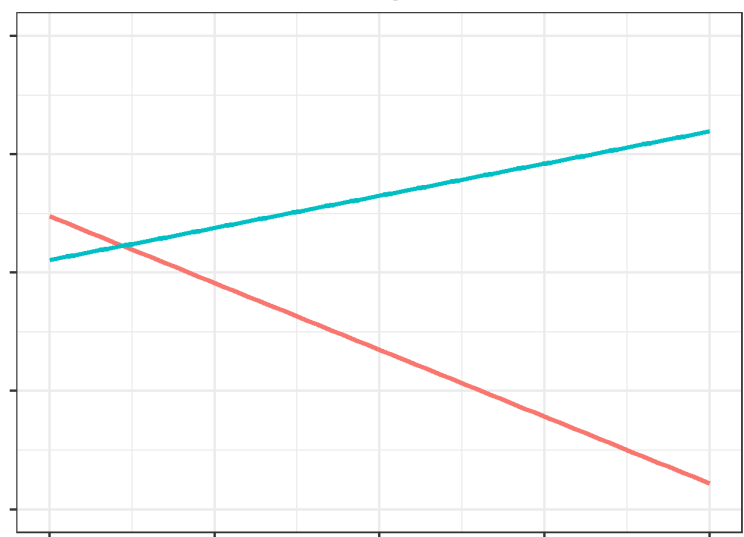

Study 3

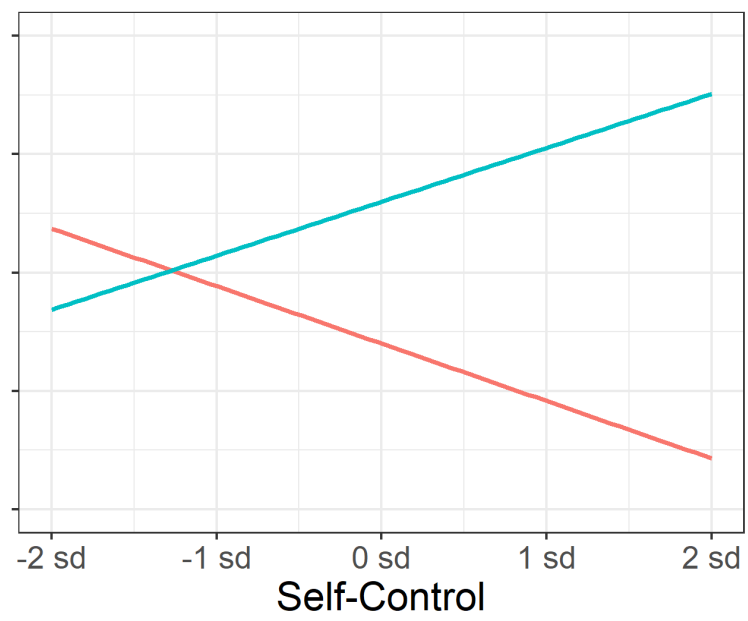

Figure 7. The interaction of behavior valence with self-control across Studies 1-all, 2, and 3. Each interaction modeled here was established in a mixed-effect regression analysis that predicted behavior regularity as a function of only valence and self-control (with all three variables standardized prior to analysis). Valence was an a priori variable that contrasted positive versus negative behaviors (Table 1). The SM provides details of the regression analysis procedure and results. 
When all factors from the Situated Action Cycle for habitual behavior were added to the original regression models, the self-control $\times$ valence interactions in Figure 7 completely disappeared across studies. When the same procedure was applied to the neuroticism $\times$ valence interactions in Figure 8, they, too, disappeared completely. These results demonstrate that factors from the Situated Action Cycle underlie the behavioral tendencies associated with self-control and neuroticism. The SM presents these analyses in detail.

We next used stepwise regression to identify the specific $\mathrm{SAM}^{2}$ factor(s) from the Situated Action Cycle that explained variance in these interactions. At each regression step, all factors were entered, one at a time, to assess how much adding each alone decreased the estimated regression coefficient for the interaction of interest. After each step, we added the (remaining) factor that decreased the coefficient the most, continuing until all factors had been added. The SM provides the details of these analyses.

Figure 9 presents results from the stepwise process for the self-control $\times$ valence interactions in Studies 1-all, 2, and 3. Across studies, automaticity, consistency, long-term reward, and immediate reward explained the increased regularity of positive behaviors and the decreased regularity of negative behaviors associated with self-control. Figure 10 presents the analogous results for the neuroticism $\times$ valence interactions. Across studies, automaticity, long-term reward, and consistency explained the decreased performance of positive behaviors and the increased performance of negative behaviors associated with neuroticism.

These analyses further demonstrate construct validity of the $\mathrm{SAM}^{2} \mathrm{HBI}$. Not only does this instrument capture well-established relations between behavior regularity and relevant personality measures, it also provides insights into factors from the Situated Action Cycle that underlie the associated behavioral dispositions.

\section{Establishing habitual behaviors}

The dimension of habitualness. We next illustrate how the $\mathrm{SAM}^{2} \mathrm{HBI}$ can be used to establish habitual behaviors for a group or for an individual. As discussed earlier, considerable disagreement surrounds the criteria that establish a behavior as a habit. For example, a habit could be a behavior produced automatically or a behavior preceded by automatic cognition. A habit could be a behavior that is performed frequently in consistent situations. A habit could be a ballistic behavior not modulated by reward. A habit could be a behavior performed regularly as conditions allow, 

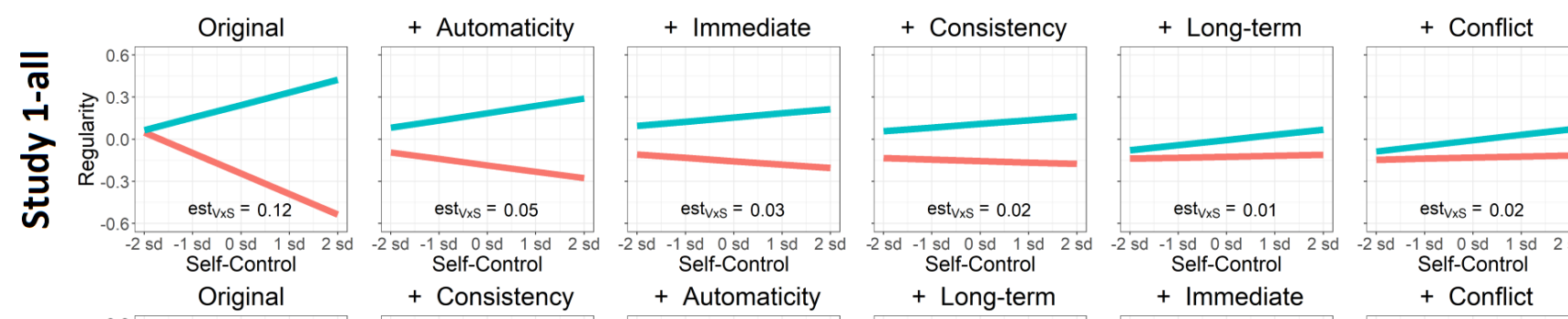

Valence

= Positive

+ Consistency

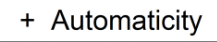

+ Long-term

+ Immediate
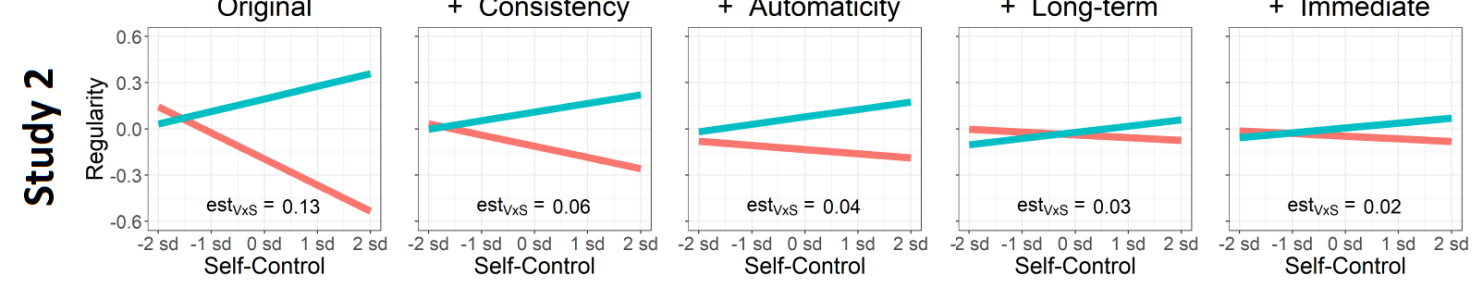

Self-Contro

Original
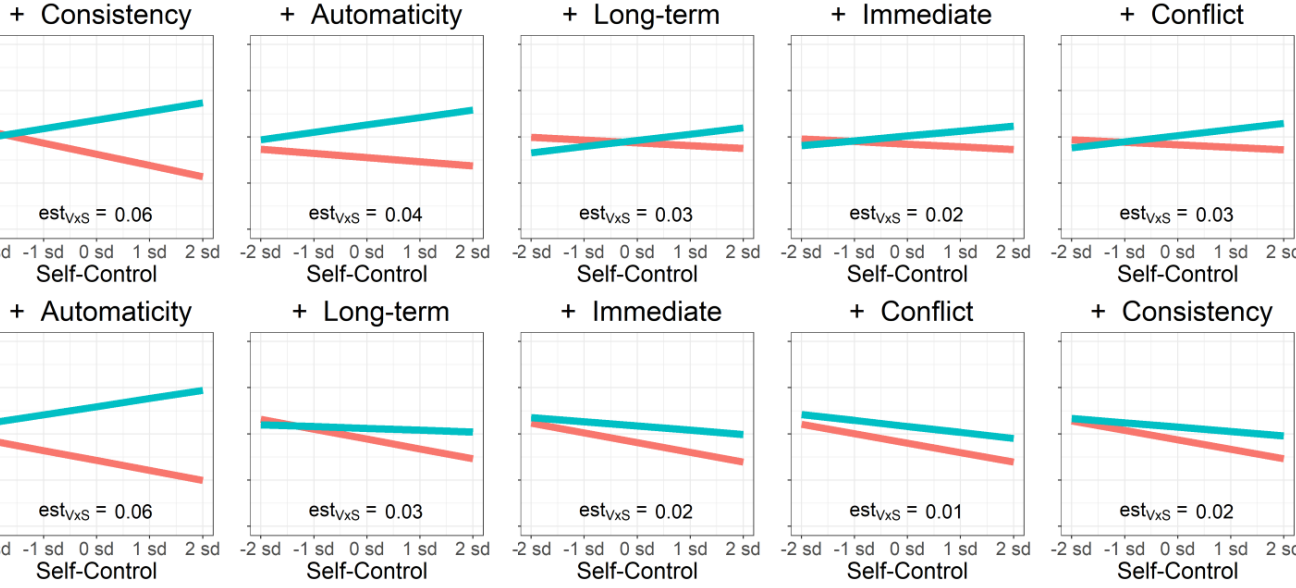

Valence

- Positive

Negative
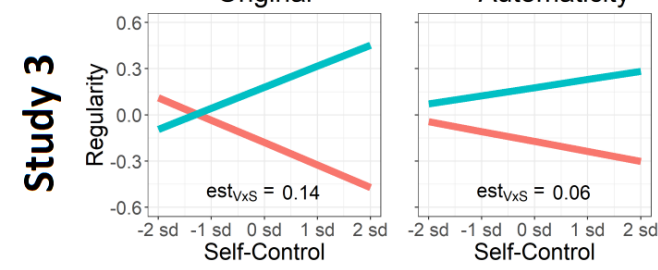

+ Consistency

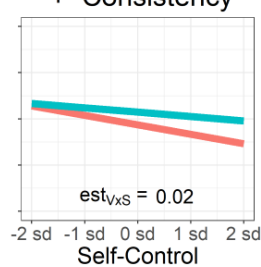

Valence

Figure 9. Results from stepwise regression to establish the $\mathrm{SAM}^{2}$ predictors that explained variance in the valence $\times$ self-control interaction in Studies 1-all, 2, and 3 (Figure 6). In the original analyses (left column), behavior regularity was regressed onto only valence and self-control in Model 2 from the analysis pipeline to test the valence $\times$ self-control interaction maximally. The five primary SAM ${ }^{2}$ predictors (Figure 1 ) were then added one at a time into the original regression to assess how much each alone decreased the coefficient for the valence $\times$ self-control interaction (est $t_{V \times S}$ ). The predictor that decreased est $v_{V \times S}$ the most is shown in the second column, along with a plot of the resulting interaction and the new value of est $v_{v \times s}$. In four further iterations of the stepwise process, the remaining $\mathrm{SAM}^{2}$ predictors were again added one by one to identify the predictor that next decreased the est ${ }_{\mathrm{V} \times \mathrm{S}}$ interaction the most. These results are shown in the third, fourth, fifth, and sixth columns. The SM provides details of the regression analysis procedure and results. 
regardless of automaticity, consistency, and reward. No doubt merit exists in all these proposals.

To establish an inclusive approach to this issue, we developed a composite measure of habitualness that combined regularity, consistency, and automaticity (i.e., the average of an individual's judgements of regularity, consistency, and automaticity for each behavior in Table 1). Essentially, this measure assumes that as behavior becomes increasingly regular, consistent, and automatic, it becomes increasingly habitual in a continuous manner.

Establishing habitualness at the group level. Table 5 presents the 80 behaviors from Table 1 ordered by composite habitualness averaged across the 199 participants in Study 2. The 40 most habitual behaviors appear on the left, and the 40 least habitual behaviors appear on the right. Examining the most and least habitual behaviors confers face validity on the composite measure of habitualness. The most habitual behaviors strike us as intuitively likely to be highly habitual (e.g., saying please and thank you, check my phone, shower every day, worry). In contrast, the least habitual behaviors strike us as intuitively likely to be less habitual (e.g., chew on pencils, skip lectures, use substances to relax, litter).

For each study, Figure 11 presents a histogram for the 80 behaviors as a function of composite habitualness. Strong similarity in the distributions holds across studies, with maximal density in the 50 to 60 range. An interesting question is where one might establish a threshold on this distribution for classifying a behavior as a habit. Because all measures going into composite habitualness used a 0 to 100 likelihood scale, a value of 50 can be viewed as a behavior that was performed regularly, automatically, and in consistent situations about $50 \%$ of the time. What should the threshold of this measure be for calling a behavior a habit? If the threshold were set at $80 \%$, very few behaviors would be habits at the group level. If the threshold were set around 50\%, many habits would be performed regularly and automatically in consistent situations $50 \%$ of the time. Should these be called habits? Many are behaviors that people might well consider habitual, such as take study breaks, eat healthy snacks, and use shopping lists.

An alternative way of viewing these plots is to conceptualize habitualness as a dimension that varies continuously across behaviors. From this perspective, attempting to establish a category of bona fide habits is not likely to be easy, successful, or theoretically meaningful. Similarly, instead of asking how long it takes to learn a habit (118), it perhaps is more useful to establish how the habitualness of a behavior grows continuously over time. 
Table 5. The 80 behaviors ordered by the composite measure of habitualness (averaged across Study 2 participants), with their composite reward values shown as well.

\begin{tabular}{|c|c|c|c|c|c|c|c|}
\hline \multirow{2}{*}{\multicolumn{2}{|c|}{ Behavior number* / name }} & \multicolumn{2}{|c|}{ Composite measure } & & & \multicolumn{2}{|c|}{ Composite measure } \\
\hline & & Habitualness & \multirow{2}{*}{$\begin{array}{c}\text { Reward } \\
3.57\end{array}$} & \multicolumn{2}{|c|}{ Behavior number* / name } & \multirow{2}{*}{$\begin{array}{r}\text { Habitualness } \\
58.07\end{array}$} & \multirow{2}{*}{$\begin{array}{r}\text { Reward } \\
1.87\end{array}$} \\
\hline 28 & Say 'please' and 'thank you' & 87.25 & & 42 & Take study breaks & & \\
\hline 39 & Check my phone multiple times a day & 85.03 & 1.31 & 54 & Pick my spots and/ or scabs & 57.64 & -0.99 \\
\hline 34 & Charge my devices & 83.57 & 2.94 & 08 & Drink soft drinks & 57.29 & 0.11 \\
\hline 50 & Cover my mouth when sneezing, coughing or yawning & 81.25 & 2.79 & 02 & Eat healthy snacks & 57.19 & 2.63 \\
\hline 27 & Hold doors open for others & 78.51 & 2.58 & 24 & Ignore my own needs & 56.65 & -1.55 \\
\hline 49 & Shower every day & 74.33 & 3.17 & 71 & Buy brand name products & 56.24 & 0.47 \\
\hline 38 & Use my phone as a social crutch & 72.77 & 1.03 & 79 & Leave plug sockets switched on & 56.24 & -1.47 \\
\hline 57 & Wash my clothes & 72.66 & 2.77 & 43 & Set goals before engaging in a task & 55.84 & 2.34 \\
\hline 03 & Eat vegetables & 72.23 & 3.08 & 67 & Use shopping lists & 54.95 & 1.73 \\
\hline 74 & Recycle & 71.80 & 2.64 & 61 & Allow messes to build up in my work area & 53.38 & -1.55 \\
\hline 22 & Worry & 71.56 & -1.82 & 33 & Make back-up copies of important documents and files & 53.23 & 2.01 \\
\hline 37 & Spend a large amount of time on social media & 71.15 & 0.25 & 52 & Go to sleep and wake up at the same times & 51.58 & 1.69 \\
\hline 75 & Reuse carrier bags & 70.82 & 2.50 & 12 & Take standing and walking breaks when sitting for long periods of time & 51.12 & 2.09 \\
\hline 73 & Turn off lights when leaving a room & 70.24 & 2.17 & 15 & Reward myself with food and/or drink after exercise & 51.08 & 0.09 \\
\hline 68 & Shop for groceries & 69.26 & 2.41 & 09 & Exercise & 50.93 & 2.18 \\
\hline 23 & Criticise myself & 69.24 & -1.13 & 16 & Use the lift instead of taking the stairs & 50.85 & 0.14 \\
\hline 25 & Maintain contact with family & 68.48 & 2.74 & 19 & Express my emotions constructively & 50.46 & 1.92 \\
\hline 40 & Use my phone whilst on the toilet & 68.28 & 0.77 & 29 & Use bad language in public & 50.42 & -1.17 \\
\hline 45 & Procrastinate & 67.90 & -0.33 & 06 & Eat dessert & 50.04 & 0.27 \\
\hline 51 & Brush my teeth twice a day & 67.48 & 2.95 & 70 & Spend to make myself feel better & 49.43 & 0.18 \\
\hline 58 & Put things back after I have finished using them & 66.42 & 2.15 & 72 & Make impulsive purchases & 48.36 & -0.22 \\
\hline 48 & Multi-task during work & 66.24 & 1.31 & 07 & Eat fast foods & 46.84 & -0.21 \\
\hline 41 & Study for my course(s) & 66.13 & 2.51 & 53 & Pick my nose & 45.56 & -0.82 \\
\hline 60 & Clean my residence & 65.60 & 2.75 & 05 & Drink alcohol & 44.22 & -0.55 \\
\hline 46 & Work whilst watching TV or listening to music & 64.64 & 0.85 & 80 & Throw away food & 43.99 & -1.87 \\
\hline 01 & Eat fruit & 63.45 & 3.07 & 69 & Dip into funds I have set aside & 43.73 & -0.84 \\
\hline 59 & Empty the bins & 63.40 & 2.02 & 56 & Bite my nails & 41.06 & -2.00 \\
\hline 17 & Take time to relax & 63.10 & 2.90 & 31 & Pay little attention to others when they are talking & 40.78 & -1.61 \\
\hline 04 & Check food labels before making purchases & 62.11 & 1.94 & 14 & Avoid long walks & 40.71 & -1.00 \\
\hline 13 & Be sedentary for long periods of time & 61.73 & -0.72 & 66 & Buy from charity and/ or second-hand shops & 38.54 & 0.74 \\
\hline 78 & Buy new condition items & 61.28 & 1.72 & 62 & Ignore stains and spills & 36.29 & -1.57 \\
\hline 63 & Leave dishes to wash later & 60.90 & -0.51 & 30 & Interrupt others & 35.64 & -2.00 \\
\hline 10 & Walk or bike when possible & 60.69 & 2.40 & 11 & Participate in sports activities and clubs & 34.79 & 1.08 \\
\hline 64 & Leave clothes lying around & 60.62 & -1.03 & 32 & Make myself the centre of conversation & 34.77 & -1.46 \\
\hline 26 & Maintain contact with friends & 60.55 & 2.90 & 55 & Chew on pencils and/ or pens & 33.04 & -2.31 \\
\hline 44 & Pack what I need the night before & 60.07 & 2.06 & 35 & Limit the amount of time each day I spend using technology & 31.90 & 0.28 \\
\hline 65 & Budget & 59.84 & 2.40 & 36 & Restrict my use of technology before sleep & 30.86 & 0.37 \\
\hline 18 & Do at least one thing a day that I enjoy and look forward $t$ & 58.79 & 2.72 & 47 & Skip lectures & 29.28 & -1.76 \\
\hline 20 & View challenges with a positive attitude & 58.47 & 2.65 & 21 & Use substances to relax & 29.22 & -1.93 \\
\hline 76 & Use reusable cups & 58.46 & 1.34 & 77 & Litter & 20.05 & -3.09 \\
\hline
\end{tabular}



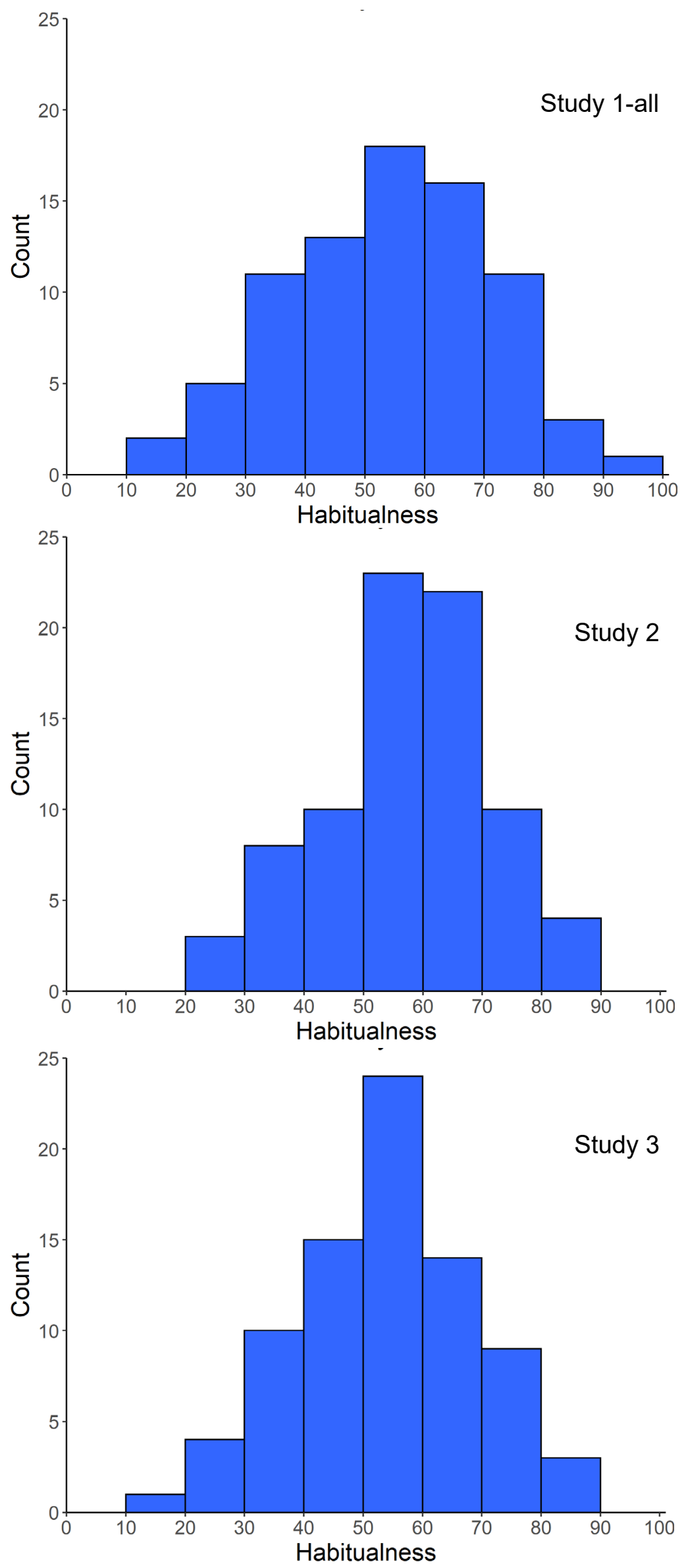

Figure 11. Histograms showing counts of the 80 behaviors in Table 1 on their compositive values for habitualness averaged across participants in Studies 1-all, 2, and 3. 
Establishing habitualness at the individual level. As we saw earlier in Figure 2 and Table 2 , considerable differences occurred in how regularly individuals performed the 80 behaviors in Table 1. Thus, the values of composite habitualness for specific behaviors are likely to vary considerably across individuals as well.

We first addressed this issue as follows. For each study, we computed an intraclass correlation that established how much the composite measure of habitualness varied between individuals (ICC2; 24,25). Analogous to Table 2, these intraclass correlations assessed interrater agreement in habitualness for the 80 behaviors. For Studies 1-all, 2, and 3, these values were .31, .26, and .27, respectively, indicating large individual differences in habitualness. Because the ICC2 estimates random effects, these values generalize to other individuals in the population.

To further document individual differences in habitualness, we established how many of the 80 behaviors in Table 1 were the first most habitual behavior for at least one individual. In Studies 1 -all, 2 , and 3, this number was 35,53 , and 39 out of 80 , respectively. We then assessed the number of behaviors that fell into the top five most habitual behaviors for at least one individual. In Studies 1-all, 2, and 3, this number was 65, 73, and 70 out of 80 respectively. Earlier we proposed that the 80 behaviors in Table 1 could potentially be highly habitual for at least some individuals. These results support that claim.

Figure 12 further illustrates these large individual differences, showing the top ten most habitual behaviors for eight individuals in Study 2. Whereas the four individuals on the left were relatively high in self-control and low in neuroticism, the four individuals on the right were relatively low in self-control and high in neuroticism. As can be seen, considerable variability existed in the top ten most habitual behaviors for even individuals with the same personality profile. Even more striking differences occurred between profiles.

For each individual, an inset figure plots the histogram of their 80 behaviors for the measure of composite habitualness (with the axes being the same as the group distributions in Figure 11). As can be seen, the individual distributions varied widely, departing considerably from the group distributions. Again, large individual differences occurred, and it is not clear how one might motivate a consistent habit threshold across them in a principled manner.

\section{Establishing the relation between habitualness and reward}

Finally, we illustrate how the $\mathrm{SAM}^{2} \mathrm{HBI}$ can be used to establish the relation between 


\section{Check my phone multiple times a day}

Brush my teeth twice a day

Participate in sports activities and clubs

Use my phone whilst on the toilet

Hold doors open for others

Recycle

Say please and thank you

$\square \rightarrow$

Budget

Turn off lights when leaving a room

Reuse carrier bags

163 Say please and thank you

Brush my teeth twice a day

Reuse carrier bags

Recycle

Walk or bike when possible

$100.00 \quad 0.65$

$98.07 \quad 3.00$

$97.27 \quad 5.00$

$\begin{array}{ll}96.87 & 3.25\end{array}$

$96.50 \quad 5.00$

$94.27 \quad 1.70$

$92.67 \quad 4.00$

$92.17 \quad 4.15$

$91.60 \quad 2.70$

$91.60 \quad 3.40$

$100.00 \quad 3.60$

$100.00 \quad 5.00$

$99.90 \quad 4.65$

$99.20 \quad 5.00$

$99.10 \quad 4.95$

Put things back after I have finished using them

$98.77 \quad 5.00$

$\begin{array}{lll}\text { Make back-up copies of important documents and files } & 96.87 & 5.00 \\ \text { Turn off lights when leaving a room } & 96.53 & 3.20\end{array}$

Pick my spots and/ or scabs

$95.37 \quad 0.00$

Eat vegetables

157 Brush my teeth twice a day

Reuse carrier bags

Use reusable cups

Eat fruit

Maintain contact with family

Express my emotions constructively

$94.13 \quad 4.80$

$99.00 \quad 5.00$

$95.37 \quad 5.00$

$85.63 \quad 3.75$

$84.83 \quad 4.95$

$84.03 \quad 5.00$

$82.87 \quad 4.75$

$82.30 \quad 1.25$

my mouth when sneezing, coughing or yawning

$79.90 \quad 3.10$

$79.43 \quad 4.70$

$78.23 \quad 1.50$

Exercise

$100.00 \quad 4.90$

$100.00 \quad 4.90$

17 Say please and thank you

Check my phone multiple times a day

Study for my course(s)

Shower every day

Use my phone as a social crutch

Multi-task during work

$100.00 \quad 5.00$

$100.00 \quad 5.00$

$99.97 \quad 5.00$

$99.90 \quad 5.00$

$99.57 \quad 3.95$

$99.43 \quad 5.00$

$99.33 \quad 4.90$

$98.97 \quad 4.65$
41 Use my phone as a social crutch

Check my phone multiple times a day

Charge my devices

Work whilst watching TV or listening to music

Bite my nails

Leave dishes to wash later

Spend a large amount of time on social media

Leave plug sockets switched on

Use my phone whilst on the toilet

Reuse carrier bags

58 Take time to relax

Maintain contact with friends

Allow messes to build up in my work area

Leave clothes lying around

Cover my mouth when sneezing, coughing or yawning

Leave dishes to wash later

Be sedentary for long periods of time

Criticise myself

Turn off lights when leaving a room

Procrastinate

84 Check food labels before making purchases

Use the lift instead of taking the stairs

Worry

Say please and thank you

Spend a large amount of time on social media

Use my phone as a social crutch

Check my phone multiple times a day

Procrastinate

Recycle

Wash my clothes

168 Drink soft drinks

Exercise

Worry

Criticise myself

Do at least one thing a day that I enjoy and look forward to

Maintain contact with family

Hold doors open for others

Say please and thank you

Spend a large amount of time on social media

Check my phone multiple times a day
$100.00 \quad 0.00$

$100.00 \quad 0.95$

$100.00 \quad 5.00$

$100.00 \quad 1.45$

$100.00 \quad 0.05$

$100.00-0.40$

$99.97 \quad 1.10$

$98.43-2.20$

$98.07-0.55$

$96.80 \quad 5.00$

$100.00 \quad 2.20$

$100.00 \quad 5.00$

$100.00 \quad 0.10$

$100.00 \quad 2.55$

$97.33 \quad 4.35$

$97.10-0.05$

$95.93-2.10$

$95.73-5.00$

$94.37 \quad 0.10$

$94.27 \quad 0.70$

$100.00 \quad 5.00$

$100.00 \quad 0.60$

$100.00 \quad-1.45$

$100.00 \quad 5.00$

$100.00 \quad 0.00$

$100.00 \quad 3.40$

$100.00 \quad 0.00$

$100.00 \quad 0.00$

$100.00 \quad 5.00$

$99.97 \quad 5.00$

$100.00 \quad 1.45$

$100.00 \quad 4.55$

$100.00 \quad-5.00$

$100.00-3.85$

$100.00 \quad 5.00$

$100.00 \quad 5.00$

$100.00 \quad 5.00$

$100.00 \quad 5.00$

$100.00 \quad 1.85$

$100.00 \quad 3.45$

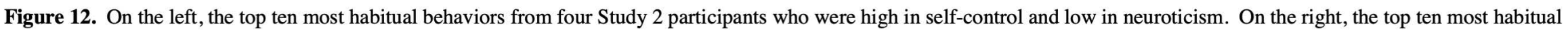

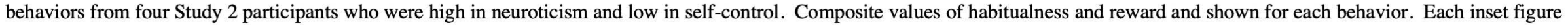
shows the habitualness histogram for a participant's 80 behaviors, with the same axes as the histograms in Figure 11 . 
habitualness and reward at both the group and individual levels. As described earlier, some researchers propose that habits run ballistically, not modulated by reward. In contrast, other researchers make empirical and theoretical cases that reward modulates the implementation of habitual behavior.

To establish the relation between habitualness and reward, we constructed a composite measure of reward from the $\mathrm{SAM}^{2}$ measures for immediate and long-term reward: the average of an individual's judgements of immediate and long-term reward for each behavior in Table 1. Table 5 presents the compositive values averaged across participants in Study 2. Figure 12 presents the compositive values for the top ten most habitual behaviors of eight participants in Study 2.

Assessing the relation between habitualness and reward at the group level. Figure 13 presents scatterplots for the composite measures of habitualness and reward across the 80 behaviors at the group level (averaged across participants in Studies 1-all, 2, and 3). The point in a scatterplot for each behavior is designated by its number in Tables 1 and 5. As can be seen, habitualness and reward were positively related across studies, exhibiting correlations of $.57, .65$., and .47 for Studies 1 -all, 2, and 3, respectively (every $p<.0001$, two-tailed). As a behavior became increasingly habitual, it became increasingly rewarding as well.

Examining specific behaviors in Figure 13 is informative. Across studies, Saying please and thank you (behavior 28) was simultaneously most habitual and most rewarding. Conversely, at the other end, littering (behavior 77) was simultaneously least habitual and least rewarding. Other interesting cases include worry (behavior 22) — a highly habitual behavior low in reward — and participate in sports activities and clubs (behavior 11) —a relatively non-habitual behavior high in reward. The position of a specific behavior within a scatterplot was strikingly constant across studies, again demonstrating the replicability of results obtained with the $\mathrm{SAM}^{2} \mathrm{HBI}$.

Assessing the relation between habitualness and reward at the individual level. We next assessed the correlation between the composite measures of habitualness and reward for each individual across the 80 behaviors. Figure 14 plots the distribution of these correlations in each study, where a point represents the correlation for one individual. Similar to the analogous correlations at the group level, habitualness tended to be positively related to reward across individuals, with mean values of .45, .48, and .41 for Studies 1-all, 2, and 3, respectively (all different from 0, two-tailed: $t=26.27, \mathrm{SE}=.02, p<.0001 ; t=32.04, \mathrm{SE}=.02, p<.0001 ; t=$ 

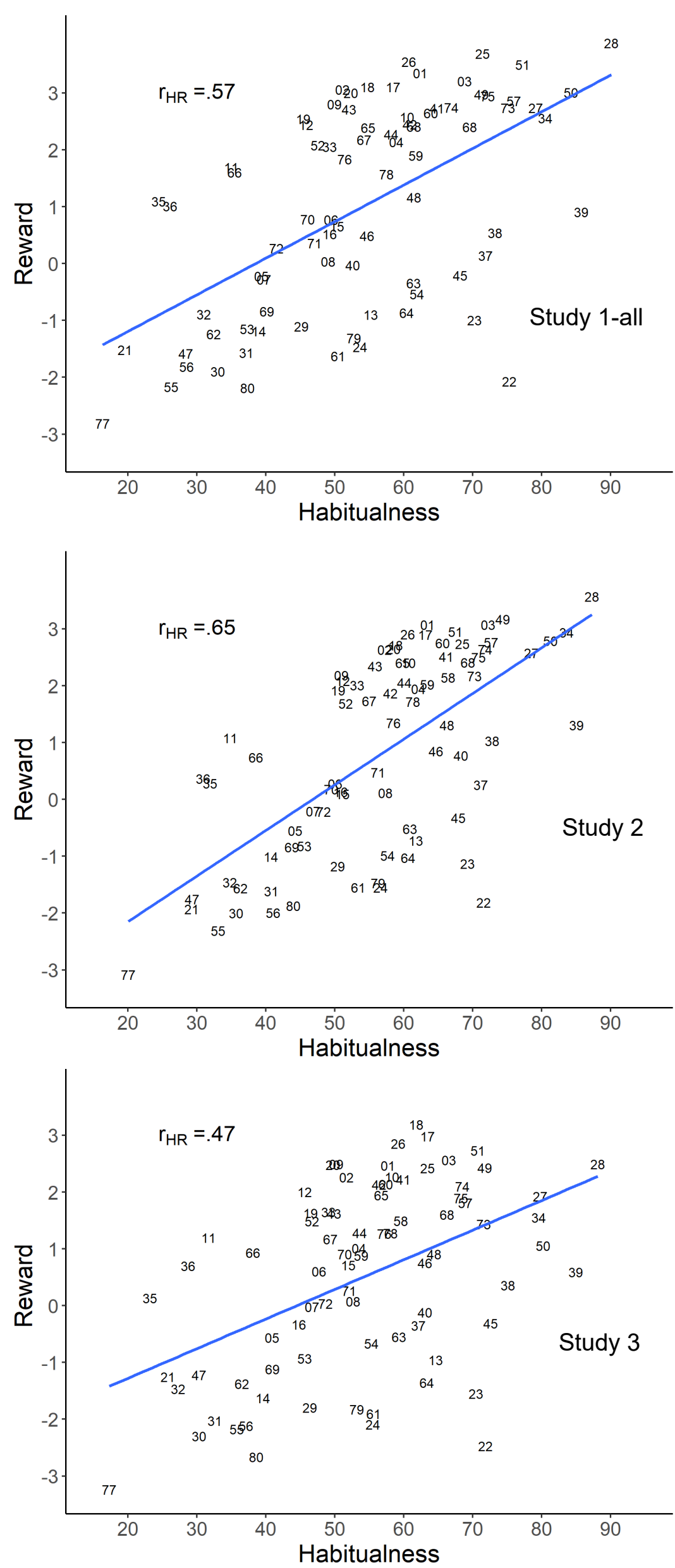

Figure 13. Scatterplots of the 80 behaviors in Table 1 on their compositive values for reward and habitualness averaged across participants in Studies 1-all, 2, and 3. The behavior numbers correspond to those in Table 1. $r_{H R}$ is the correlation between the two composite means across behaviors. 
16.86, $\mathrm{SE}=.02, p<.0001)$.

Finally, we assessed whether individual correlations of habitualness and reward were related to the personality measures of self-control and neuroticism. As Figure 15 illustrates, a consistent pattern emerged across studies. As an individual's self-control increased, the positive relation between reward and habitualness tended to become stronger, exhibiting positive correlations of $.51, .48$, and .41 for Studies 1-all, 2, and 3, respectively (every $p<.0001$, two-tailed). In contrast, as an individual's neuroticism increased, the positive relation between reward and habitualness tended to become weaker, exhibiting negative correlations of -.38, -.40., and -.39 for Studies 1-all, 2, and 3, respectively (every $p<.0001$, two-tailed). These results suggest that self-control was associated with increased reward monitoring, whereas neuroticism was associated with decreased reward monitoring.

\section{Discussion}

From the theoretical perspectives of grounded, situated, and embodied cognition, we developed a new approach for assessing individual differences - the Situated Assessment Method $\left(\mathrm{SAM}^{2}\right)$. Rather than asking individuals to abstract across situations when evaluating a target construct, $\mathrm{SAM}^{2}$ situates the construct in two ways: First, $\mathrm{SAM}^{2}$ assesses the construct in situations where it occurs. Second, SAM ${ }^{2}$ assesses factors from the Situated Action Cycle that potentially influence the construct in these situations. The result is a rich descriptive profile for each individual that embeds the construct in their unique situational experience of it.

\section{The SAM ${ }^{2}$ Habitual Behavior Instrument (SAM ${ }^{2}$ HBI): Summary of results}

Three studies demonstrated that the $\mathrm{SAM}^{2} \mathrm{HBI}$ produces highly replicable findings. The same robust pattern of results that occurred in the larger samples of Studies 2 and 3 ( $n=199$ and $n$ $=115$, respectively) also occurred in samples as small as $\mathrm{n}=31$ (Studies $1 \mathrm{a}$ and $1 \mathrm{~b}$ in Study 1-all). This pattern also replicated regardless of whether $\mathrm{SAM}^{2}$ judgments were collected in the laboratory (Studies 1a and 1b) or online (Studies 1c, 2, and 3), whether judgments were collected in a fixed order (Studies 1-all and 2) or in a random order (Study 3), or whether additional measures were collected (Study 2). We next summarize the pattern of results that replicated across studies.

Trait-level measures. The SAM${ }^{2} \mathrm{HBI}$ produced a variety of trait-level measures that provided insight into behavior regularity at both the group and individual levels. Most basically, the SAM ${ }^{2}$ HBI produced a trait-level measure of behavior regularity, showing that, on average, participants performed the 80 behaviors in Table 1 about $50 \%$ of the time when possible (with some behaviors 

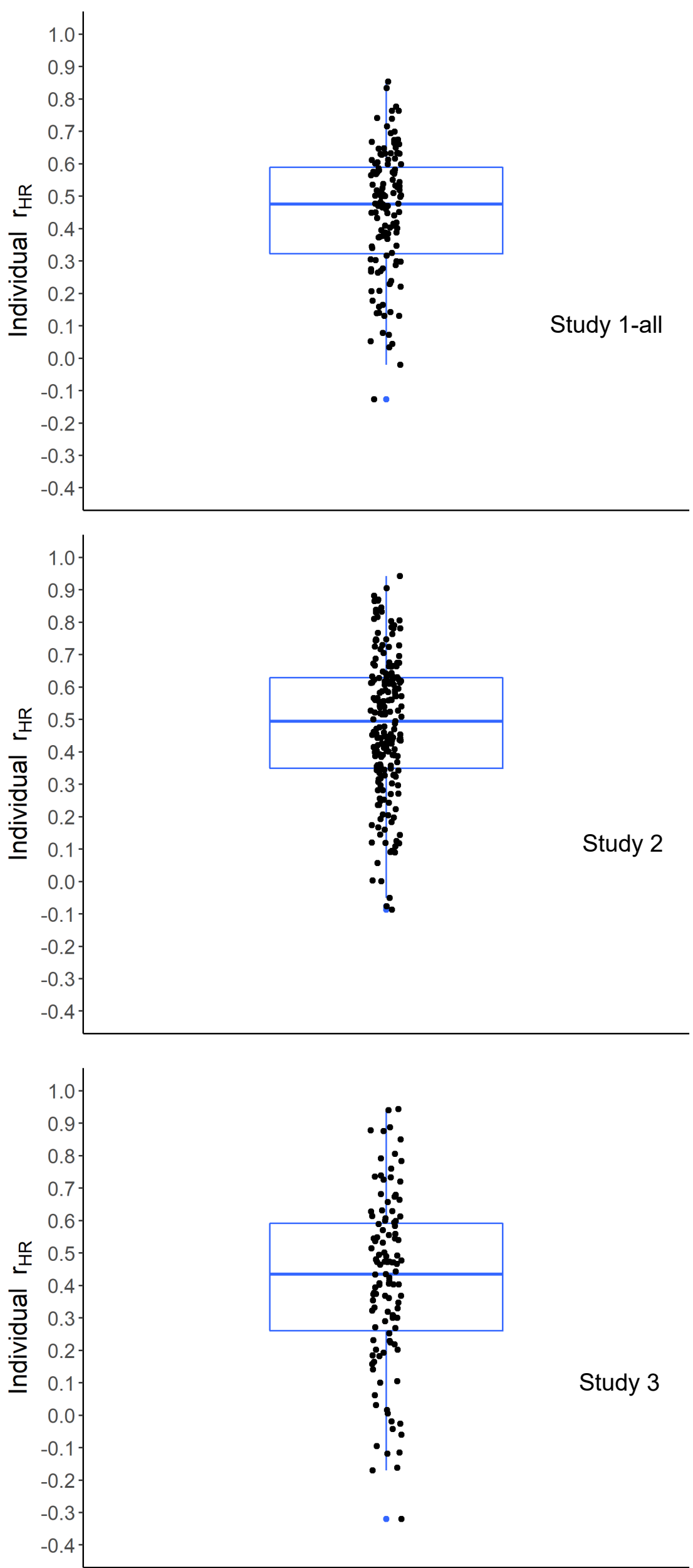

Figure 14. Values of the correlation between the composite measures of habitualness and reward, $\mathrm{r}_{\mathrm{HR}}$, for individual participants in Studies 1-all, 2, and 3. 

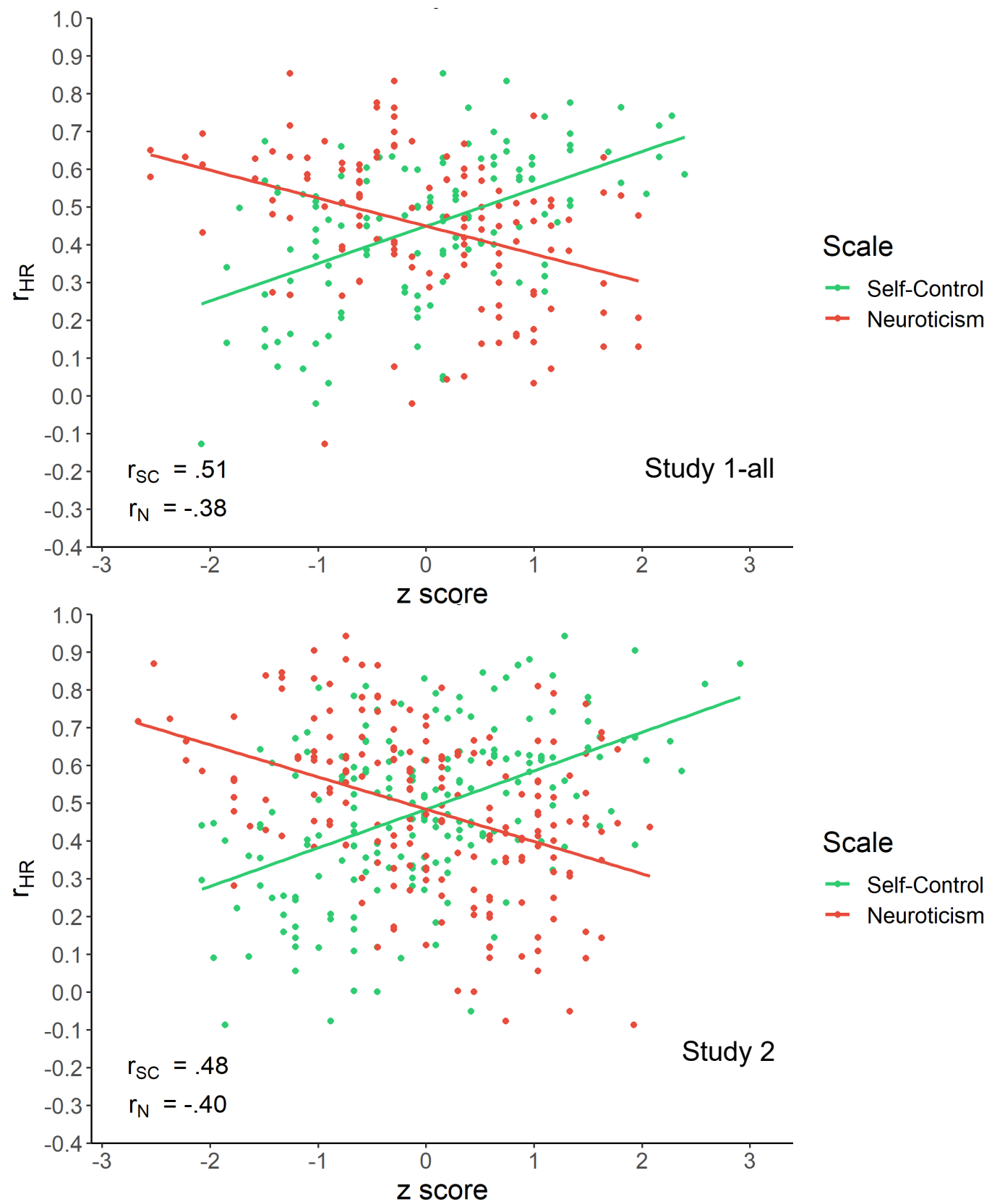

\section{Scale}

$\rightarrow$ Self-Control

$\rightarrow$ Neuroticism

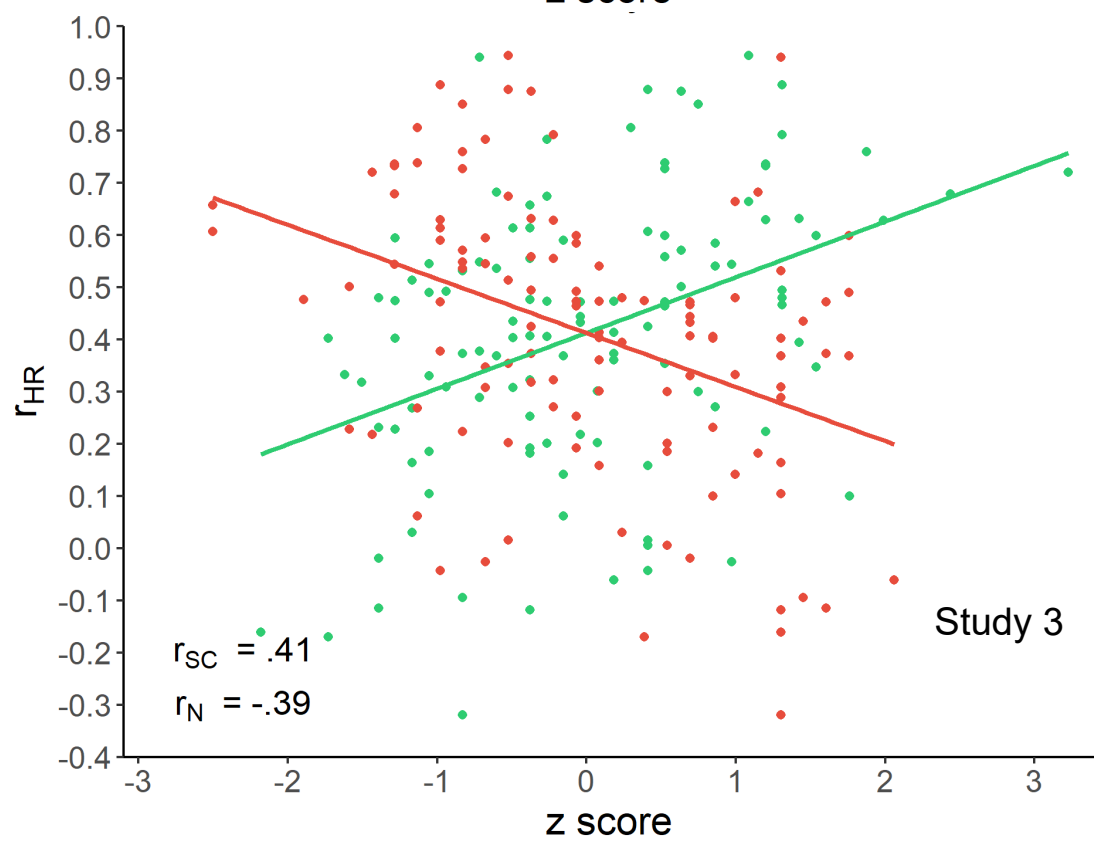

Scale

$\rightarrow$ Self-Control

$\rightarrow$ Neuroticism

Figure 15. Individual correlations in Studies 1-all, 2, and3 between the composite measures of habitualness and reward, $r_{\mathrm{HR}}$, plotted as a function of individual measures for self-control and neuroticism (both transformed to $\mathrm{z}$ scores plotted along the $\mathrm{X}$ axis). 
performed more regularly and others performed less). More specific trait-level measures for the regularity of positive behaviors and for the regularity of negative behaviors captured personality differences in self-control and neuroticism (Figures 7 and 8).

Finally, a composite measure of habitualness established habitual behaviors at the group and individual levels (Figures 11 and 12), and a composite measure of reward assessed relations between habitualness and reward (Figures 13,14,15). By combining measures from Figure 1 and Table 2 in other ways, additional measures that capture other facets of habitual behavior could be constructed.

Large individual differences. Participants exhibited considerable differences in the behaviors they performed regularly. When we assessed interrater reliability between participants in how regularly they performed the 80 behaviors, we only observed agreement around .30 (Table 2). For a composite measure of habitualness, participants similarly only exhibited agreement around .28. Behaviors that were habitual for some participants were not habitual for others. These results indicate that the $\mathrm{SAM}^{2} \mathrm{HBI}$ captured large individual differences in habitual behavior. Consistent with the classic interactionist perspective $(38-40,45,48)$, an individual's behavior regularity varied considerably across situations, with the pattern of variability taking different forms for different individuals (i.e., the individual $\times$ behavior interaction in Figure 2).

Although such variability could simply reflect noise in judgments of regularity, individual regressions explained a median variance of approximately $75 \%$ in individual regularity judgments (Figure 6). Such a high level of explained variance suggests that individual regularity judgments were highly systematic — not noisy. If low interrater agreement had simply reflected noise, such high percentages of explained variance at the individual level would not have been expected.

Test reliability. When Cronbach's alpha was used to assess test reliability, acceptable values around .80 and above were observed (Table 3). Interestingly, the 80 behaviors in the overall measure of behavior regularity exhibited relatively low coherence around .04, indicating that individual behaviors ordered participants quite differently. As we suggested, such low coherence most likely resulted from attempting to sample behaviors representatively. Broad coverage ensured that an overall measure of regularity reflected as many potentially relevant behaviors as possible.

Including a large number of 80 behaviors, however, compensated for low coherence, producing satisfactory values of alpha (consistent with the Spearman-Brown formula; 23-26). When coherence was computed separately for positive and negative behaviors, alpha increased as 
the behaviors within each group became more coherent (to around .11). As each subset of behaviors became more similar, the resulting coherence increased alpha, even though the number of behaviors decreased from 80 to 40 (i.e., increased coherence had greater impact on alpha than decreased behaviors).

Construct validity with respect to the Situated Action Cycle. When behavior regularity was regressed onto factors from the Situated Action Cycle associated with habitual behavior, multilevel mixed-effect regressions generally explained approximately $65 \%$ of the total variance at the group level. This explanatory success demonstrates content validity of the $\mathrm{SAM}^{2} \mathrm{HBI}$ : Its measures across the Situated Action Cycle are sufficiently thorough and complete to explain the bulk of the variance in habitual behavior.

Two additional findings consistent with the habits literature offered construct validity for the $\mathrm{SAM}^{2}$ measure of behavior regularity (Figure 4). First, regularity was most highly associated with factors that reflect conditioning - consistency and automaticity. Second, immediate and long-term reward were also associated with regularity, albeit to a lesser extent. Because the measure of SAM ${ }^{2}$ behavior regularity captured this well-established pattern of results, it again exhibits construct validity. Rather than establishing a measure of behavior regularity in isolation, the $\mathrm{SAM}^{2} \mathrm{HBI}$ embedded it in other measures from the Situated Action Cycle. As a result, relations between regularity and related situational factors emerged.

Regressions performed at the individual level were even more successful in establishing construct validity. As just noted, individual regressions explained more variance in regularity than did group-level regressions, with the median explained variance around 75\% (Figure 6). Because regularity exhibited such large individual differences, individual regressions explained more variance than group regressions, where individual differences attenuated explained variance. Explanatory success at the individual level again demonstrates content validity, with factors from the Situated Action Cycle explaining the bulk of the variance in behavior regularity.

Similar to the group-level regressions, regularity in the individual regressions tended to be best predicted by consistency and automaticity, with immediate and long-term reward making lesser contributions (Table 4). Nevertheless, substantial individual differences occurred, with different individuals exhibiting different patterns of prediction (Figure 5). As these patterns illustrate, the $\mathrm{SAM}^{2} \mathrm{HBI}$ established individual models of behavior regularity, specifying factors from the 
Situated Action Cycle that best predicted regularity for each individual. By assessing factors from the Situated Action Cycle across behaviors, the SAM ${ }^{2}$ HBI not only established a situated measure of behavior regularity for each individual, it also constructed a unique predictive model of the situational factors that influence it.

Validity with respect to self-control and neuroticism. Interactions of behavior valence with self-control and neuroticism further established the validity of the SAM ${ }^{2} \mathrm{HBI}$ (Figures 7 and 8). In every study, increasing self-control was associated with increasing regularity of positive behaviors and decreasing regularity of negative behaviors (a self-control $\times$ valence interaction). Conversely, increasing neuroticism was associated with decreasing regularity of positive behaviors and increasing regularity of negative behaviors (a neuroticism $\times$ valence interaction). The fact that the $\mathrm{SAM}^{2}$ measure of behavior regularity captured these well-established interactions between personality and valence further demonstrates its construct validity.

Additional construct validity follows from $\mathrm{SAM}^{2}$, s ability to explain these interactions with factors from the Situated Action Cycle. As Figures 9 and 10 illustrate, automaticity, consistency, long-term reward, and immediate reward explained the variance in the interactions between valence, self-control, and neuroticism (across all three studies). These results suggest that selfcontrol and neuroticism can be understood as emerging from individual patterns in these basic cognitive-affective processes $(38-40,45,48)$.

Establishing the habitualness of behaviors. As described earlier, various factors have been used to define habitualness in the literature, including frequency, consistency, and automaticity. To capture the importance of all these factors here, we established a composite measure of a behavior's habitualness that averaged its regularity (related to frequency), consistency, and automaticity. We then used this composite measure to establish the habitualness of behaviors at the group and individual levels.

The composite measure exhibited high face validity at the group level (Table 5). Behaviors that intuitively appear high in habitualness received the highest values; behaviors that intuitively appear low received the lowest values. When histograms were plotted of these compositive values, the distributions exhibited highest density in the center (Figure 11). Most behaviors exhibited moderate levels of habitualness, with fewer exhibiting high or low levels. Interestingly, however, many of the behaviors in Table 1 were highly habitual for at least some participants, again 
demonstrating considerable individual differences (also see Figure 12).

To assess the relation of reward to habitualness, we computed a composite measure of reward (the average of immediate and long-term reward). When we plotted the relation of habitualness to reward, a strong positive relation emerged at the group level (Figure 13). A similar pattern occurred at the individual level, together with large individual differences (Figure 14). Notably, the correlation between reward and habitualness took opposing forms as self-control versus neuroticism increased (Figure 15). As self-control increased, the positive relation between reward and habitualness tended to become stronger; as neuroticism increased, the positive relation between reward and habitualness tended to become weaker.

\section{Limitations}

Correlational results. As informative as these results are in assessing habitual behaviors at the individual and group levels, their correlational nature does not support causal interpretations. Indeed, one must be careful to avoid the temptation of drawing causal conclusions from the rich descriptive data available. Responsible speculation about causality, however, can still be useful. On the one hand, correlational evidence can contribute to the accumulation of evidence related to causal claims, being consistent or inconsistent with them. On the other, correlational evidence is typically easier to gather than causal evidence and can thus serve as an effective form of discovery, to be followed later by careful experimental assessment when possible and useful.

Population sampled. Another limitation of the current findings is that they were obtained from UK students aged 18-30. It is important to explore whether the $\mathrm{SAM}^{2} \mathrm{HBI}$ exhibits similar results in other samples. Although we are optimistic that it will generalize robustly, verification is necessary. To the extent that different populations exhibit different habitual behaviors, the behaviors in Table 1 must be adapted. To the extent that relevant factors from the Situated Action Cycle vary, they may need to be adapted as well (Figure 1). We assume, however, that other implementations are likely to reproduce many features of the results reported here. Specifically, we anticipate that behavior regularity will exhibit similar relations to conditioning, reward, and personality, along with a comparable range of individual differences.

Subjective vs. objective measures. We assessed self-reports of the measures listed in Figure 1 and Table 2, not objective measurements. Specifically, we assessed how participants perceive processes like automaticity and immediate reward in their behavior. Certainly, self-report 
can be relatively accurate, especially when specific situational assessments are made (50-53). Furthermore, these judgments exhibited considerable construct validity with respect to the Situated Action Cycle and in relation to personality variables. Nevertheless, it is essential to bear in mind that participants' perceived assessments of the measures in Figure 1 and Table 2 constitute the data reported here.

Although we established construct validity for these measures, it remains to be shown that they actually track their real-world counterparts. Thus, an important direction for future research is to establish the accuracy of the specific measures collected here (and also in much other research on habitual behavior). We hasten to add that rigorously assessing constructs such as regularity, automaticity, consistency, immediate reward, and long-term reward in the real-world constitutes a significant challenge for habits research. Laboratory conditioning paradigms offer one approach for measuring some of these constructs more objectively. Importantly, however, such paradigms can never fully substitute for measuring these constructs in natural everyday behavior. Resolving these issues continues to constitute a major challenge. We believe that the $\mathrm{SAM}^{2} \mathrm{HBI}$ offers a reasonable approach to approximating the measurement of relevant constructs in an effective and informative manner, notwithstanding the limitations of self-report.

Test duration. The $\mathrm{SAM}^{2} \mathrm{HBI}$ assessed here took participants a little under an hour to complete (including additional assessments of Self-Control and the Five-Factor Inventory). Certainly, this is not an unreasonable amount of time to spend, especially as an exercise in a clinical setting. Yet, relative to many other assessment instruments, an hour is a long time. In the next section, we suggest alternative forms that brief $\mathrm{SAM}^{2}$ instruments could take, should a brief form be appropriate in a clinical or research setting.

\section{Developing SAM${ }^{2}$ assessment instruments}

The $\mathrm{SAM}^{2}$ framework is sufficiently open-ended to support a wide variety of instruments for diverse purposes. We next explore a few of the possibilities.

Full-length $\mathbf{S A M}^{2}$ instruments. In contexts where a thorough assessment of a construct is desired, a relatively comprehensive and representative set of situations may be optimal, along with a thorough sampling of relevant factors from the Situated Action Cycle. The SAM ${ }^{2}$ HBI explored here constitutes an example.

If one wanted to develop a version of the $\mathrm{SAM}^{2} \mathrm{HBI}$ for another population, one could 
sample behaviors and factors from the Situated Action Cycle differently. To assess habitual behavior in children across levels of SES, for example, one could sample behaviors related to education, play, and family, along with factors from the Situated Action Cycle related to resources, supervision, and choice. Alternatively, to assess habitual behavior in socially anxious adults, one could focus on social and achievement behaviors, along with factors from the Situated Action Cycle related to threat, social support, and acceptance.

More generally, SAM${ }^{2}$ instruments can be developed for many other constructs besides habitual behaviors. In our work, we have explored instruments for stress, emotion, trichotillomania, social connectedness, eating, drinking, emotion regulation, mindfulness, wellbeing, and sustainability. In each case, we first establish relevant situations where the target construct occurs, often informed by empirical norming. We then establish a measure of the construct. Finally, we examine the relevant scientific literature to identify factors from the Situated Action Cycle that influence the construct in the situations of interest. We then build a $\mathrm{SAM}^{2}$ instrument around this content. Typically, we find that such instruments take 30 to 60 minutes for participants to perform, depending on the domain. Again, we assume that instruments like these may typically be most relevant in clinical or research settings.

Brief SAM ${ }^{2}$ instruments. We nevertheless appreciate the need for briefer instruments in other contexts. One brief instrument that may often be sufficient is to only obtain judgements of the target construct across situations and to not collect judgments for influential factors from the Situated Action Cycle. To the extent that a larger initial study has validated judgments of the construct with respect to these factors, such judgments alone may be sufficient for many purposes.

Consider the SAM ${ }^{2} \mathrm{HBI}$ developed here. A brief version would only include regularity judgments of the 80 situations (taking 5-10 minutes). We now know that regularity judgments exhibit construct validity because we validated them here in relation to factors from the Situated Action Cycle and to personality measures. We also know that constructing separate overall traitlevel measures for the regularity of positive vs. negative behaviors can be informative, making them potentially applicable in a variety of clinical and applied settings.

A second way to create a brief $\mathrm{SAM}^{2}$ instrument is to compress either or both dimensions of situatedness: (a) situations, (b) factors from the Situated Action Cycle. To compress situations, cluster analyses could establish clusters of related situations, with the most central situation 
sampled for each cluster. Another possibility for a habitual behavior instrument would be to sample one positive behavior and one negative behavior from each of the domains in Table 1, reducing the number of behaviors assessed from 80 to 20 . To compress factors from the Situated Action Cycle, a set of existing factors, such as those in Figure 1, could be submitted to exploratory factor analysis. Again, groups of closely related measures from the Situated Action Cycle could be identified, with only one from each group included in a brief instrument.

In this manner, brief compressed versions of full-length instruments can be developed. It is an empirical question whether a brief version is as informative as a complete version. In clinical settings, another interesting question is whether using a brief version leads to comparable insight and behavior change in clients as using a complete version.

Targeted SAM${ }^{2}$ instruments. A different approach to creating brief instruments is to focus on a target construct and then to only sample situations and factors from the Situated Action Cycle relevant to this focus. In the domain of habitual behavior, for example, one could focus on health behaviors, social behaviors, or sustainable behaviors. For each focus, a smaller set of behaviors becomes relevant, and perhaps also fewer factors from the Situated Action Cycle.

Another approach is to create a brief $\mathrm{SAM}^{2}$ instrument targeted to a specific individual. By only including situations and factors relevant for the individual, a brief instrument for individual use can be created. In the domain of habitual behaviors, for example, a brief unique instrument for each individual could be developed that tracks their positive and negative behaviors over time. Such instruments might also be useful in experience sampling applications.

Clinical use. $\mathrm{SAM}^{2}$ instruments could be designed to support a variety clinical functions. Most basically, such instruments could map out a client's habitual tendencies and identify factors from the Situated Action Cycle likely to influence them. In particular, such instruments could establish the regularity of performing positive versus negative behaviors (or other target behaviors of interest). A predictive model could then be developed of factors from the Situated Action Cycle that influence these behaviors, with this model then being used to guide behavior change interventions. Finally, longitudinal assessment could track the effectiveness of these interventions over time.

\section{Implications for issues surrounding habitual behavior}

The construct of habitualness. As described earlier, considerable debate continues to address the issue of what constitutes a habit. One key issue is whether automaticity defines habitualness. If 
so, is automaticity necessary at the stage of behavioral execution, during prior cognitive processing, or both? A second key issue is whether situational consistency is a necessary feature of a habit. A third is whether habits are ballistic stimulus-response patterns, uninfluenced by reward.

Several results reported here bear on these issues. As Figure 5 illustrates, the automaticity of executing a behavior was not strongly associated with behavior regularity for all participants, nor was consistency. These results question whether automaticity and consistency are universal features of habitual behavior. As Figure 5 further illustrates, the predictive models for individual participants varied widely. Although clusters of individuals exhibited similar profiles of the factors that predict regularity, considerable differences existed between clusters. These large individual differences in predictive models further suggest caution in trying to develop a narrow definition of habitual behavior.

Still another problem is the distributions of habitualness in Figures 11 and 12. On the one hand, it's not clear where one can effectively set a threshold that distinguishes habits from nonhabits. On the other, many behaviors fall in the middle of the distribution, where behaviors exhibit regularity, consistency, and automaticity a little over $50 \%$ of the time. Many of these behaviors could well be habitual, at least to some extent.

Together, these results suggest that a definition of habits based on necessary features such as exhibiting automaticity and consistency may not be feasible or theoretically desirable. Instead, the construct of habitualness may be better understood as a family resemblance structure, having prototypical features that are not definitional $(119,120)$. From this perspective, habitual behaviors are statistically likely to be automatic, consistent, and rewarding, with none of these features being necessary. Because habitualness takes so many different forms, attempting to understand how its complex multidimensional structure manifests across individuals and behaviors may be more productive than attempting to establish a rigid definition. Similar conclusions have been reached about the construct of automaticity (121-123).

Finally, it may be useful to view this heterogeneous construct of habitualness as a continuous dimension. Rather than attempting to distinguish categories of habits from non-habits, it may be more useful to ask how habitual a behavior is, where habitualness tends to be continuously associated with increasing regularity, consistency, and automaticity. Individual behaviors for individual people are likely to vary widely on these factors, reflecting both personal and situational constraints. 
The relation of reward to habitualness. A common view is that a behavior only becomes a habit if it is elicited ballistically, no longer modulated by reward. In their classic review of the nonhuman conditioning literature, Yin and Knowlton (106) document how organisms generally monitor reward and adjust behavior accordingly, with increases in reward increasing response rates to obtain reward (and vice versa). Reward only falls away at the very end of the conditioning process, following extreme levels of practice. Researchers often argue that humans never reach these levels, with reward always continuing to modulate behavior $(87,89,97,101)$. Recent reviews document extensive evidence for the ever-present role of reward in habitual behavior, along with compelling theoretical arguments for why we should expect this $(88,92,95)$.

Several of our results bear on this issue. First, immediate and long-term reward were consistently related to behavior regularity at both the group and individual levels (Table 4, Figures 4 and 5). Notably, however, both forms of reward were less related to regularity than were consistency and automaticity, suggesting that conditioned responses played stronger roles in habitual behavior than did adaptive reward monitoring. Nevertheless, reward was consistently related to regularity.

Second, considerable individual differences emerged in the importance of reward. As Figure 5 illustrated for Study 2, immediate reward was more important for some individuals, whereas longterm reward was more important for others (with figures in the SM showing similar patterns for Studies 1-all and 3). Some form of reward was typically important across individuals (both in many cases). Again, such results question a rigid definition of what constitutes a habit, with the role of reward also likely to vary widely across specific behaviors for specific individuals.

Third, and perhaps most importantly, habitualness and reward were positively related at both the group and individual levels (Figures 13 and 14). As a behavior became more habitual, it became more rewarding. This consistent pattern challenges the widespread assumption that reward becomes increasingly non-influential as behaviors become increasingly habitual. To the contrary, a different process may occur: Increasing reward for a behavior may increase habitualness, thereby increasing the likelihood of receiving high rewards.

Finally, the relation between reward and habitualness behaved quite differently as selfcontrol vs. neuroticism varied (Figure 15). As self-control increased, the positive relation between reward and habitualness increased as well. One speculative interpretation is that individuals high in self-control closely monitor sources of reward in their experience. When they find a reward of 
interest, they regularize the behavior needed to obtain it (i.e., the basic idea behind instrumental conditioning; 68). Furthermore, as a reward becomes more attractive, they strengthen habitual responding to ensure that the reward is obtained (i.e., the widespread finding that organisms monitor reward and adjust behavior accordingly; 95,106). Conversely, as reward for a behavior decreases, individuals high in self-control weaken their tendency to perform the behavior. Although tendencies may remain to perform it, these individuals extinguish to the extent that reward ends. Most importantly, these individuals allow reward to inform their behavior, performing it to the extent that it's rewarding. Of course, our correlational data don't establish this causal relationship. It would be interesting in future work, though, to assess the causal relation between high self-control and reward monitoring.

In contrast, the positive relation between reward and habitualness decreased as neuroticism increased (Figure 15). One speculative possibility is that the strong emotional reactions associated with neuroticism deplete the regulatory resources available for monitoring reward and adapting behavior as reward changes (124). As a consequence, behavior becomes increasingly ballistic as poorly monitored behaviors become rigidly automated in response to situational cues. Again, further research is required to examine this possibility.

Clinical implications. To the extent that reward motivates the regularization of behavior, clinical implications follow. To change behavior, it is first necessary to establish compelling new rewards that motivate individuals to restructure their behavior $(51,125,126)$. To increase the habitualness of a positive behavior in Table 5, find a new reward that motivates this increase. To decrease the habitualness of a negative behavior, remove the reward that is holding it in place. By controlling reward, the behavior can be moved up or down the regression line between reward and habitualness in Figure 13. By monitoring behavior regularity and its relation to reward, the $\mathrm{SAM}^{2}$ HBI could be used to guide habit change in clinical settings.

\section{Conclusion}

Inspired by grounded, situated, and embodied approaches to cognition, the Situated Assessment Method $\left(\mathrm{SAM}^{2}\right)$ establishes individual differences in a construct across the situations where it occurs, also capturing factors from the Situated Action Cycle that influence it. In the implementation for habitual behaviors developed here, we saw that this approach produces useful measures of a behavior regularity that are reliable, valid, and informative. For each individual, a 
detailed profile of habitual behavior also emerges. The general $\mathrm{SAM}^{2}$ framework can be implemented in diverse ways to achieve a wide variety of assessment goals.

\section{Acknowledgements}

We are grateful to the Healthy Cognition Research Group in the School of Psychology and Neuroscience at the University of Glasgow for ongoing contributions, support, and advice on this project. We are also grateful to Daniel Cervone for directing us to relevant literature on personality and for insightful discussion about it. 


\section{References}

1. Aydede M, Robbins P. The Cambridge handbook of situated cognition. Cambridge: Cambridge University Press; 2009.

2. Barsalou LW. Perceptual symbol systems. Behav Brain Sci. 1999;22:577-660.

3. Barsalou LW. Grounded cognition. Annu Rev Psychol. 2008;59:617-45.

4. Barsalou LW. Simulation, situated conceptualization, and prediction. Philos Trans R Soc B Biol Sci. 2009 May 12;364(1521):1281-9.

5. Barsalou LW. Situated conceptualization: Theory and applications. In: Foundations of embodied cognition: Volume 1 Perceptual and emotional embodiment. East Sussex: Psychology Press; 2016. p. 11-37.

6. Barsalou LW. Situated conceptualization offers a theoretical account of social priming. Curr Opin Psychol. 2016 Dec;12:6-11.

7. Coello Y, Fischer MH, editors. Foundations of embodied cognition: Volume 1. Perceptual and emotional embodiment. Oxford, UK: Routledge; 2016.

8. Coello Y, Fischer MH, editors. Foundations of embodied cognition: Volume 2. Conceptual and interactive embodiment. Oxford, UK: Routledge; 2016.

9. Newen A, Bruin LD, Gallagher S, editors. The Oxford Handbook of 4E Cognition. Oxford, New York: Oxford University Press; 2018. 960 p. (Oxford Library of Psychology).

10. Pecher D, Zwaan RA, editors. Grounding cognition: The role of perception and action in memory, language, and thinking. New York: Cambridge University Press; 2005. 336 p.

11. McCrae RR, Costa Jr. PT. The Five-Factor Theory of personality. In: Handbook of personality: Theory and research. New York: Guilford; 1999. p. 139-53.

12. Costa Jr. PT, McCrae RR. Domains and Facets: Hierarchical Personality Assessment Using the Revised NEO Personality Inventory. J Pers Assess. 1995 Feb;64(1):21-50.

13. Goldberg LR. An alternative 'description of personality': the big-five factor structure. J Pers Soc Psychol. 1990;

14. John OP, Srivastava S. The Big Five trait taxonomy: History, measurement, and theoretical perspectives. In: Handbook of personality: Theory and research, 2 [Internet]. New York: Guilford; 1999 [cited 2017 May 3]. p. 102-38. Available from: http://www.rc.usf.edu/ jdorio/Personality/BIG\%20FIVE\%20TRAIT\%20TAXONOMY.pdf

15. Tangney JP, Baumeister RF, Boone AL. High Self-Control Predicts Good Adjustment, Less Pathology, Better Grades, and Interpersonal Success. J Pers. 2004 Apr;72(2):271-324.

16. Cohen S, Kamarck T, Mermelstein R. A global measure of perceived stress. J Health Soc Behav. 1983 Dec;24:385.

17. Watson D, Anna L, Tellegen A. Development and Validation of Brief Measures of Positive and Negative Affect: The PANAS Scales. J Pers Soc Psychol. 1988;54:1063-70.

18. Diener E, Emmons RA, Larsen RJ, Griffin S. The Satisfaction With Life Scale. J Pers Assess. 1985 Feb 1;49(1):71-5.

19. Blandine de Lauzon, Romon M, Deschamps V, Lafay L, Borys J-M, Karlsson J, et al. The Three-Factor Eating Questionnaire-R18 Is Able to Distinguish among Different Eating 
Patterns in a General Population. J Nutr. 2004 Sep 1;134(9):2372-80.

20. Baer RA. Using Self-Report Assessment Methods to Explore Facets of Mindfulness. Assessment. 2006 Mar 1;13(1):27-45.

21. Branson V, Dry MJ, Palmer E, Turnbull D. The Adolescent Distress-Eustress Scale: Development and Validation. SAGE Open. 2019 Jul 1;9(3):2158244019865802.

22. Peel R, Caltabiano N. The relationship sabotage scale: an evaluation of factor analyses and constructive validity. BMC Psychol. 2021 Sep 19;9(1):146.

23. Cronbach LJ. Coefficient alpha and the internal structure of tests. Psychometrika. 1951 Sep $1 ; 16(3): 297-334$.

24. de Vet HCW, Mokkink LB, Mosmuller DG, Terwee CB. Spearman-Brown prophecy formula and Cronbach's alpha: different faces of reliability and opportunities for new applications. J Clin Epidemiol. 2017 May;85:45-9.

25. Shrout PE, Fleiss JL. Intraclass correlations: Uses in assessing rater reliability. Psychol Bull. 1979 Mar;86(2):420-8.

26. Bloch R, Norman G. Generalizability theory for the perplexed: A practical introduction and guide: AMEE Guide No. 68. Med Teach. 2012 Nov;34(11):960-92.

27. Allen MJ, Yen WM. Introduction to measurement theory. Monterey, CA: Brooks/Cole; 2001. $330 \mathrm{p}$.

28. Cronbach LJ, Meehl PE. Construct validity in psychological tests. Psychol Bull. 1955;52:281-302.

29. Campbell DT, Fiske DW. Convergent and discriminant validation by the multitraitmultimethod matrix. Psychol Bull. 1959;56:81-105.

30. Ajzen I. Intuitive theories of events and the effects of base-rate information on prediction. $\mathbf{J}$ Pers Soc Psychol. 1977;35(5):303-14.

31. Gelman SA, Legare CH. Concepts and Folk Theories. Annu Rev Anthropol. 2011 Oct 21;40(1):379-98.

32. Nisbett RE, Wilson TD. Telling more than we can know: Verbal reports on mental processes. Psychol Rev. 1977;84(3):231.

33. Ross L. The Intuitive Psychologist And His Shortcomings: Distortions in the Attribution Process. In: Berkowitz L, editor. Advances in Experimental Social Psychology. Academic Press; 1977. p. 173-220.

34. Wilson TD. Strangers to ourselves. Cambridge, MA: Harvard University Press; 2004.

35. Tversky A, Kahneman D. Availability: A heuristic for judging frequency and probability. Cognit Psychol. 1973 Sep 1;5(2):207-32.

36. Wänke M, Schwarz N, Bless H. The availability heuristic revisited: Experienced ease of retrieval in mundane frequency estimates. Acta Psychol (Amst). 1995 Jun 1;89(1):83-90.

37. Fleeson W, Gallagher MP. The Implications of Big-Five Standing for the Distribution of Trait Manifestation in Behavior: Fifteen Experience-Sampling Studies and a Meta-Analysis. J Pers Soc Psychol. 2009 Dec;97(6):1097-114.

38. Fleeson W, Jayawickreme E. Whole traits: Revealing the social-cognitive mechanisms constituting personality's central variable. In: Advances in Experimental Social Psychology 
[Internet]. Elsevier; 2021 [cited 2021 Nov 25]. p. 69-128. Available from: https://linkinghub.elsevier.com/retrieve/pii/S0065260120300277

39. Bandura A. The self system in reciprocal determinism. Am Psychol. 1978;33(4):344-58.

40. Cervone D. Personality Architecture: Within-Person Structures and Processes. Annu Rev Psychol. 2005 Feb;56:423-52.

41. Cervone D, Shadel WG, Jencius S. Social-Cognitive Theory of personality assessment. Personal Soc Psychol Rev. 2001 Feb;5(1):33-51.

42. Collins MD, Jackson CJ, Walker BR, O'Connor PJ, Gardiner E. Integrating the contextappropriate balanced attention model and reinforcement sensitivity theory: Towards a domain-general personality process model. Psychol Bull. 2017 Jan;143(1):91-106.

43. Mischel W. Personality and assessment. New York: Wiley; 1968.

44. Mischel W. Toward a cognitive social learning reconceptualization of personality. Psychol Rev. 1973;80:252-83.

45. Mischel W, Shoda Y. A Cognitive-Affective System Theory of Personality:

Reconceptualizing Situations, Dispositions, Dynamics, and Invariance in Personality Structure. Psychol Rev. 1995;102:246-68.

46. Fleeson W. Toward a Structure- and Process-Integrated View of Personality: Traits as Density Distributions of States. J Pers Soc Psychol. 2001;80:1011-27.

47. Fleeson W. Moving Personality Beyond the Person-Situation Debate: The Challenge and the Opportunity of Within-Person Variability. Curr Dir Psychol Sci. 2004 Apr;13(2):83-7.

48. Endler NS, Magnusson D. Toward an interactional psychology of personality. Psychol Bull. 1976;83(5):956-74.

49. Reynolds K, Turner J, Branscombe N, Mavor K, Bizumic B, Subasic E. Interactionism in Personality and Social Psychology: An Integrated Approach to Understanding the Mind and Behaviour. Eur J Personal. 2010 Aug 1;24:458-82.

50. Ajzen I, Fishbein M. Attitude-behavior relations : A theoretical analysis and review of empirical research. Psychol Bull. 1977;84:888-918.

51. Ajzen I, Fishbein M. The influence of attitudes on behavior. In: The handbook of attitudes. Mahwah, New Jersey: Erlbaum; 2005. p. 173-221.

52. Glasman LR, Albarracín D. Forming attitudes that predict future behavior: A meta-analysis of the attitude-behavior relation. Psychol Bull. 2006;132(5):778-822.

53. Siegel JT, Navarro MA, Tan CN, Hyde MK. Attitude-behavior consistency, the principle of compatibility, and organ donation: A classic innovation. Health Psychol. 2014;33(9):108491.

54. Costa Jr. PT, McCrae RR. The Revised NEO Personality Inventory (NEO-PI-R). In: The SAGE handbook of personality theory and assessment, Vol 2: Personality measurement and testing. Thousand Oaks, CA, US: Sage Publications, Inc; 2008. p. 179-98.

55. Cohen S, Gianaros PJ, Manuck SB. A Stage Model of Stress and Disease. Perspect Psychol Sci. 2016 Jul 1;11(4):456-63.

56. Monroe SM. Modern approaches to conceptualizing and measuring human life stress. Annu Rev Clin Psychol. 2008 Apr;4:33-52. 
57. Charles ST, Piazza JR, Mogle J, Sliwinski MJ, Almeida DM. The Wear and Tear of Daily Stressors on Mental Health. Psychol Sci. 2013 Mar 26;24(5):733-41.

58. Pangallo A, Zibarras L, Lewis R, Flaxman P. Resilience through the lens of interactionism: A systematic review. Psychol Assess. 2015 Mar;27(1):1-20.

59. Barsalou LW. Establishing generalizable mechanisms. Psychol Inq. 2019 Oct 2;30(4):220 30 .

60. Brunswik E. Representative design and probabilistic theory in a functional psychology. Psychol Rev. 1955 May;62(3):193-217.

61. Dhami MK, Hertwig R, Hoffrage U. The Role of Representative Design in an Ecological Approach to Cognition. Psychol Bull. 2004;130(6):959-88.

62. Hammond KR, Stewart TR. The Essential Brunswik: Beginnings, Explications, Applications. Oxford: Oxford University Press; 2001. 562 p.

63. Miller LC, Shaikh SJ, Jeong DC, Wang L, Gillig TK, Godoy CG, et al. Causal Inference in Generalizable Environments: Systematic Representative Design. Psychol Inq. 2019 Oct 2;30(4):173-202.

64. Yang Y, Read SJ, Denson TF, Xu Y, Zhang J, Pedersen WC. The Key Ingredients of Personality Traits: Situations, Behaviors, and Explanations. Pers Soc Psychol Bull. 2014 Jan;40(1):79-91.

65. Zayas V, Whitsett DD, Lee JJY, Wilson N, Shoda Y. From situation assessment to personality: Building a social-cognitive model of a person. In: Handbook of personality theory and testing. Thousand Oaks, CA, US: Sage; 2008. p. 377-401.

66. Barsalou LW. Challenges and opportunities for grounding cognition. J Cogn. 2020;3(1):31.

67. Bouton ME, Todd TP. A fundamental role for context in instrumental learning and extinction. Behav Processes. 2014 May;104:13-9.

68. Domjan M. The principles of learning and behavior. Independence, KY: Cengage Learning; 2014. 450 p.

69. Miller GA, Galanter E, Pribram KH. Plans and the structure of behavior. New York: Holt Reinhart \& Winston; 1960. 226 p.

70. Newell A, Simon HA. Human problem solving. Englewood Cliffs, NJ: Prentice-Hall; 1972.

71. Anderson JR. The architecture of cognition. Cambridge, MA: Cambridge University Press; 1983.

72. Newell A. Production systems: Models of control structures. In: Visual Information Processing [Internet]. Academic Press; 1973 [cited 2020 May 26]. p. 463-526. Available from: http://www.sciencedirect.com/science/article/pii/B9780121701505500160

73. Daw ND, Frank MJ. Reinforcement learning and higher level cognition: Introduction to special issue. Cognition. 2009 Dec;113(3):259-61.

74. Sutton RS, Barto AG. Introduction to reinforcement learning [Internet]. MIT Press; 1998 [cited 2014 Nov 21]. Available from: http://dl.acm.org/citation.cfm?id=551283

75. Botvinick MM, Niv Y, Barto AC. Hierarchically organized behavior and its neural foundations: A reinforcement learning perspective. Cognition. 2009 Dec;113(3):262-80.

76. Ito M, Doya K. Multiple representations and algorithms for reinforcement learning in the 
cortico-basal ganglia circuit. Curr Opin Neurobiol. 2011 Jun 1;21(3):368-73.

77. O'Doherty JP. Beyond simple reinforcement learning: the computational neurobiology of reward-learning and valuation. Eur J Neurosci. 2012;35(7):987-90.

78. Baerger DR, McAdams DP. Life story coherence and its relation to psychological wellbeing. Narrat Inq. 1999;9(1):69-96.

79. Edson Escalas J. Narrative processing: Building consumer connections to brands. J Consum Psychol. 2004;14(1):168-80.

80. Reese E, Haden CA, Baker-Ward L, Bauer P, Fivush R, Ornstein PA. Coherence of personal narratives across the lifespan: A multidimensional model and coding method. J Cogn Dev. 2011 Oct 1;12(4):424-62.

81. Stein NL, Hernandez MW. Assessing understanding and appraisal during emotional experience. In: Handbook of emotion elicitation and assessment. Oxford: Oxford University Press; 2007. p. 298-317.

82. Barsalou LW. Situated simulation in the human conceptual system. Lang Cogn Process. 2003;18:513-62.

83. Barsalou LW, Dutriaux L, Scheepers C. Moving beyond the distinction between concrete and abstract concepts. Philos Trans R Soc B Biol Sci. 2018 Aug 5;373(1752):20170144.

84. Epstein S. The stability of behavior: I. On predicting most of the people much of the time. J Pers Soc Psychol. 1979;37(7):1097-126.

85. Epstein S. The stability of behavior: II. Implications for psychological research. Am Psychol. 1980 Sep;35(9):790-806.

86. Fleeson W. Situation-Based Contingencies Underlying Trait-Content Manifestation in Behavior. J Pers. 2007 Aug;75(4):825-62.

87. Danner UN, Aarts H, Vries NK. Habit vs. intention in the prediction of future behaviour: The role of frequency, context stability and mental accessibility of past behaviour. Br J Soc Psychol. 2008 Jun;47(2):245-65.

88. De Houwer J. On How Definitions of Habits Can Complicate Habit Research. Front Psychol. 2019;10:2642.

89. de Wit S, Kindt M, Knot SL, Verhoeven AAC, Robbins TW, Gasull-Camos J, et al. Shifting the balance between goals and habits: Five failures in experimental habit induction. J Exp Psychol Gen. 2018 Jul;147(7):1043-65.

90. Gardner B. Habit as automaticity, not frequency. Eur Health Psychol. 2012;14:32-6.

91. Gardner B. A review and analysis of the use of 'habit'in understanding, predicting and influencing health-related behaviour. Health Psychol Rev. 2015;9(3):277-95.

92. Hommel B, Wiers RW. Towards a Unitary Approach to Human Action Control. Trends Cogn Sci. 2017 Dec 1;21(12):940-9.

93. Ji MF, Wood W. Purchase and Consumption Habits: Not Necessarily What You Intend. J Consum Psychol. 2007 Oct 1;17(4):261-76.

94. Judah G, Gardner B, Kenward MG, DeStavola B, Aunger R. Exploratory study of the impact of perceived reward on habit formation. BMC Psychol. 2018 Dec;6(1):62.

95. Kruglanski AW, Szumowska E. Habitual Behavior Is Goal-Driven. Perspect Psychol Sci. 
2020 Sep;15(5):1256-71.

96. Mazar A, Wood W. Defining Habit in Psychology. In: Verplanken B, editor. The Psychology of Habit: Theory, Mechanisms, Change, and Contexts [Internet]. New York: Springer; 2018 [cited 2019 Jul 9]. p. 13-29. Available from: https://doi.org/10.1007/978-3319-97529-0 2

97. McCarthy MB, Collins AM, Flaherty SJ, McCarthy SN. Healthy eating habit: A role for goals, identity, and self-control? Psychol Mark. 2017 Aug;34(8):772-85.

98. Ouellette JA, Wood W. Habit and intention in everyday life: The multiple processes by which past behavior predicts future behavior. Psychol Bull. 1998;124(1):54-74.

99. Verplanken B, Orbell S. Reflections on Past Behavior: A Self-Report Index of Habit Strength1. J Appl Soc Psychol. 2003;33(6):1313-30.

100. Wood W, Neal DT. The habitual consumer. J Consum Psychol. 2009 Oct;19(4):579-92.

101. Marien H, Custers R, Aarts H. Studying Human Habits in Societal Context: Examining Support for a Basic Stimulus-Response Mechanism. Curr Dir Psychol Sci. 2019;614-8.

102. McCloskey K, Johnson BT. Habits, Quick and Easy: Perceived Complexity Moderates the Associations of Contextual Stability and Rewards With Behavioral Automaticity. Front Psychol. 2019 Jul 24;10:1556.

103. Orbell S, Verplanken B. Progress and prospects in habit research. In: The psychology of habit: Theory, mechanisms, change, and context. Switzerland: Springer Nature; 2018. p. $397-410$.

104. Schneider W, Shiffrin RM. Controlled and automatic human information processing: I. Detection, search, and attention. Psychol Rev. 1977;84(1):1-66.

105. Shiffrin RM, Schneider W. Controlled and automatic human information processing: II. Perceptual learning, automatic attending and a general theory. Psychol Rev. 1977;84(2):127-90.

106. Yin HH, Knowlton BJ. The role of the basal ganglia in habit formation. Nat Rev Neurosci. 2006 Jun;7(6):464-76.

107. Hofmann W, Rauch W, Gawronski B. And deplete us not into temptation: Automatic attitudes, dietary restraint, and self-regulatory resources as determinants of eating behavior. J Exp Soc Psychol. 2007 May;43(3):497-504.

108. Hofmann W, Schmeichel BJ, Baddeley AD. Executive functions and self-regulation. Trends Cogn Sci. 2012 Mar;16:174-80.

109. Neal DT, Wood W, Wu M, Kurlander D. The Pull of the Past When Do Habits Persist Despite Conflict With Motives? Pers Soc Psychol Bull. 2011;37(11):1428-37.

110. Wood W. Habit in Personality and Social Psychology. Personal Soc Psychol Rev. 2017 Nov;21(4):389-403.

111. Adriaanse MA, Kroese FM, Gillebaart M, De Ridder DTD. Effortless inhibition: habit mediates the relation between self-control and unhealthy snack consumption. Front Psychol. 2014 May 16;5:444.

112. de Ridder DTD, Lensvelt-Mulders G, Finkenauer C, Stok FM, Baumeister RF. Taking Stock of Self-Control: A Meta-Analysis of How Trait Self-Control Relates to a Wide Range of Behaviors. Personal Soc Psychol Rev. 2012 Feb;16(1):76-99. 
113. Galla BM, Duckworth AL. More than resisting temptation: Beneficial habits mediate the relationship between self-control and positive life outcomes. J Pers Soc Psychol. 2015 Sep;109(3):508-25.

114. Duggan KA, Friedman HS, McDevitt EA, Mednick SC. Personality and Healthy Sleep: The Importance of Conscientiousness and Neuroticism. Arias-Carrion O, editor. PLoS ONE. 2014 Mar 20;9(3):e90628.

115. Elliston KG, Ferguson SG, Schüz B. Personal and situational predictors of everyday snacking: An application of temporal self-regulation theory. Br J Health Psychol. 2017 Nov;22(4):854-71.

116. Keller C, Siegrist M. Does personality influence eating styles and food choices? Direct and indirect effects. Appetite. 2015 Jan 1;84:128-38.

117. Terracciano A, Löckenhoff CE, Crum RM, Bienvenu OJ, Costa PT. Five-Factor Model personality profiles of drug users. BMC Psychiatry. 2008 Dec;8(1):22.

118. Lally P, van Jaarsveld CHM, Potts HWW, Wardle J. How are habits formed: Modelling habit formation in the real world. Eur J Soc Psychol. 2010 Oct;40(6):998-1009.

119. Rosch E, Mervis CB. Family resemblances: Studies in the internal structure of categories. Cognit Psychol. 1975;7:573-605.

120. Wittgenstein L. Philosophical investigations. Oxford: Basil Blackwell; 1953.

121. Lebois LAM, Wilson-Mendenhall CD, Barsalou LW. Are automatic conceptual cores the gold standard of semantic processing? The context-dependence of spatial meaning in grounded congruency effects. Cogn Sci. 2015;39:1764-801.

122. Moors A, De Houwer J. Automaticity: A Theoretical and Conceptual Analysis. Psychol Bull. 2006;132(2):297-326.

123. Sharma L, Markon KE, Clark LA. Toward a theory of distinct types of 'impulsive' behaviors: A meta-analysis of self-report and behavioral measures. Psychol Bull. 2014 Mar;140(2):374-408.

124. Crow AJD. Associations Between Neuroticism and Executive Function Outcomes: Response Inhibition and Sustained Attention on a Continuous Performance Test. Percept Mot Skills. 2019 Aug 1;126(4):623-38.

125. Giles EL, Robalino S, Sniehotta FF, Adams J, McColl E. Acceptability of financial incentives for encouraging uptake of healthy behaviours: A critical review using systematic methods. Prev Med. 2015 Apr 1;73:145-58.

126. Michie S, Richardson M, Johnston M, Abraham C, Francis J, Hardeman W, et al. The Behavior Change Technique Taxonomy (v1) of 93 Hierarchically Clustered Techniques: Building an International Consensus for the Reporting of Behavior Change Interventions. Ann Behav Med. 2013 Aug 1;46(1):81-95. 


\section{Supporting Information}

S1. Supplemental Material (SM)

\section{Author contributions}

LB and NC developed the original paradigm as NC's honors thesis at the University of Glasgow (Study 1a). LB, NC, EP, and CS subsequently developed Studies 1b, 1c, and 2 as replications and extensions of Study 1a. LB, NC, LD, EP, and CS subsequently developed Study 3 as NC's masters thesis. LB developed the initial analysis pipeline, with expert advice from CS. LD enhanced it considerably and implemented all final analyses for all studies, with minor assistance from LB and expert advice from CS. NC collected the data for all studies, together with LB and LD. LB and LD drafted the manuscript, and all authors contributed to its revision. All authors approved the final manuscript.

\section{Ethics Approval}

The Ethics Committee for the College of Science and Engineering at the University of Glasgow granted ethics approval for this work on 1 August 2017 (Application 300160200).

\section{Financial Disclosure Statement}

This project received support from the College of Science and Engineering at the University of Glasgow to LB for staff research and to NC for MSc research. This project also received support from Wellcome Trust grant 219390/Z/19/Z to LB (PI) and CS (co-PI), from Economic and Social Research Council grant ES/R005419/1 to EP (PI), and from Economic and Social Research Council grant ES/T011343/1 to EP (PI) and LB (co-PI). The funders had no role in study design, data collection and analysis, decision to publish, or preparation of the manuscript.

\section{Competing Interests Statement}

The authors declare that they have no competing interests. 


\section{Supplemental Material}

\section{The Situated Assessment Method (SAM²): Establishing Individual Differences in Habitual Behavior}

Léo Dutriaux ${ }^{1}$ Naomi E. Clark ${ }^{1}$ Esther K. Papies ${ }^{1}$ Christoph Scheepers ${ }^{1}$ Lawrence W. Barsalou ${ }^{1}$

${ }^{1}$ School of Psychology and Neuroscience, University of Glasgow

Léo Dutriaux is now at the Center for Mind/Brain Science, University of Trento

Naomi Clark is now at the School of Health and Life Sciences, Glasgow Caledonian University

\section{Screens for collecting judgments across studies}

together with the associated rationale and supporting data ................................. 3

Figure SM-1. Screens for collecting judgments in Study 1 -all........................................... 4

Figure SM-2. Screens for collecting judgments in Study 2 ................................................. 5

Figure SM-3. Screens for collecting judgments in Study 3 ............................................... 6

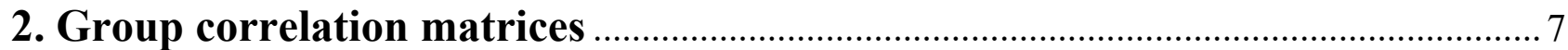

Table SM-1. Correlation matrices in Studies 1-all, 2, and 3 ................................................. 8

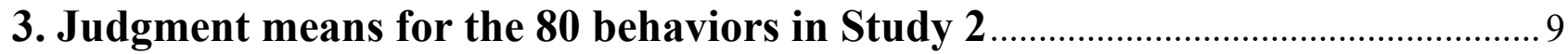

Table SM-2. Average SAM ${ }^{2}$ judgements for each behavior in Study 2 ............................... 10

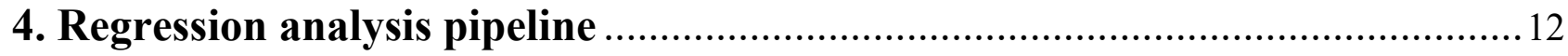

5. Assessing the validity of the a priori valence assignments ..................................... 14

Figure SM-4. Rated valence judgments for the 80 behaviors as a function of a priori valence. 15

Table SM-3. Regression results for predicting a priori and rated valence in Study 2 ............... 16

6. Clustered heatmaps for individual $\times$ behavior interactions …………….............. 17

Figure SM-5. Individual $\times$ behavior interaction in Study 1-all .............................................. 18

Figure SM-6. Individual $\times$ behavior interaction in Study 3 ……………………………....... 19

7. Regression results for the group-level analyses of Studies 1-all, 2, and 3........20

Table SM-4. Regression results for predicting regularity in Study 1-all ..................................21

Table SM-5. Regression results for predicting regularity in Study 2 ……………………......23

Table SM-6. Regression results for predicting regularity in Study 3 ........................................25

8. Clustered heatmaps of individual predictive correlations...................................2

Figure SM-7. Clustered heatmap of individual predictive correlations in Study 1-all...............28

Figure SM-8. Clustered heatmap of individual predictive correlations in Study 3 ....................29

9. Regression analyses that assessed self-control and neuroticism .........................30

Table SM-7. Regression results for assessing interactions of valence and personality .............. 31

10. Stepwise regressions to establish processes from the Situated Action Cycle that underlie self-control and neuroticism.....................................................32

Figure SM-9. Valence $\times$ self-control interactions for full models in Studies 1-all, 2, and 3......33 Figure SM-10. Valence $\times$ neuroticism interactions for full models in Studies 1-all, 2, and 3 ...34 Table SM-8. Stepwise regressions to explain the valence $\times$ self-control interactions..................35 Table SM-9. Stepwise regressions to explain the valence $\times$ neuroticism interactions ................36

11. Valence $\times$ personality interactions using rated valence in Study $2 \ldots \ldots \ldots \ldots \ldots \ldots . . . .37$

Figure SM-11. Valence $\times$ personality interactions for a priori and rated valence in Study 2 .... 38 
12. Analysis of social approval as a predictor of behavior regularity 39 Figure SM-12. Regression coefficients for predicting regularity and motivation in Study 2.....40 Table SM-10. Regression results for regularity in Study 2, including social approval ..............41

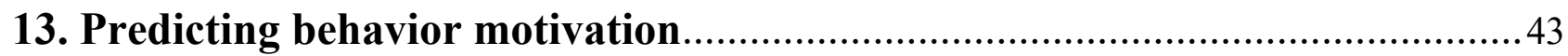

Table SM-11. Regression results for predicting behavior motivation in Study 2 .................... 44

14. Assessing explicit awareness of factors that predict behavior regularity .......47

Figure SM-13. Screen for collecting meta-cognitive prediction estimates in Study 3 ................48

Figure SM-14. Correlations between estimated and actual predictive correlations ....................49 


\section{Screens for collecting judgments across studies together with the associated rationale and supporting data}

\section{Examples of screens used to collect judgments in Studies 1-all, 2, and 3}

Figure SM-1 illustrates the 3 screens used to collect judgments in Study 1-all. Figure SM-2 illustrates the 4 screens used to collect judgments in Study 2. Figure SM-3 illustrates the 6 screens used to collect judgments in Study 3. The rationale for using these various screen formats is presented below, along with results that bear on their use.

\section{Rationale for using blocked judgments in Studies 1-all and 2}

In Study 1-all, we implemented the six judgments as two pairs of judgments in a fixed sequence of three blocks for three reasons: First, collecting two judgments in each of three blocks significantly decreased the total time required to perform the study, relative to the six blocks that would have been required for performing one judgment per block.

Second, we believed that performing related pairs of judgments would help participants perceive the differences between them, thereby producing more accurate judgments. Specifically, we assumed that judging regularity and consistency together would help participants discriminate the difference between how regularly a behavior occurs versus how consistently it occurs in the same situation. Similarly, we assumed that judging immediate and long-term reward together would help participants discriminate these two kinds of reward.

Third, we assumed that judging conflict and automaticity together would help participants perceive the difference between effortfully deciding to perform a behavior versus performing it with little thought. Instructions for each pair of judgments helped participants discriminate the two judgments.

Finally, we believed that judging the six scales in a fixed order made the task more intuitive for participants. Specifically, it seemed most natural to begin with generally assessing the regularly of performing a behavior and the consistency of the situations where it is performed. It then seemed intuitive to have participants focus on contrasting the immediate reward and long-term reward of the behavior. Finally, it seemed natural to have participants conclude with assessing the conflict associated with performing the behavior (perhaps associated with the contrast between immediate and long-term reward), followed by assessing how automatically they perform it.

\section{Results that bear on use of blocked judgments}

As the main article and the SM document extensively, the same general pattern of results occurred for Studies 1-all and 2 (where blocked judgments were collected) and for Study 3 (where individual randomized judgments were collected). The effects of the personality variables and the prediction of behavior regularity remained remarkably constant across all these studies at both the group and individual levels. Additionally, blocking did not alter the general patterns observed for the intraclass correlations, correlation matrices, and individual difference data.

As Table SM-11 illustrates for pairs of measures collected together in Studies 1-all and 2, blocked data collection had little effect compared to individual randomized data collection in Study 3. First, the correlation between regularity and consistency was actually slightly higher when they were collected separately in Study 3 (.71) than when they were collected together in Studies 1-all and 2 (.68 and .66, respectively). Second, the correlations between immediate and long-term reward in Studies 1-all and 2 (.33 and .46, respectively) were comparable to the correlation in Study 3 (.27). Third, the correlations between conflict and automaticity in Studies 1-all and 2 (-.04 and .03, respectively) were comparable to the correlation in Study 3 (.01).

Together these results indicate that blocking judgments together had little impact on the results obtained. 
Figure SM-1. Examples of the three screens used to collect judgments in Study 1-all.

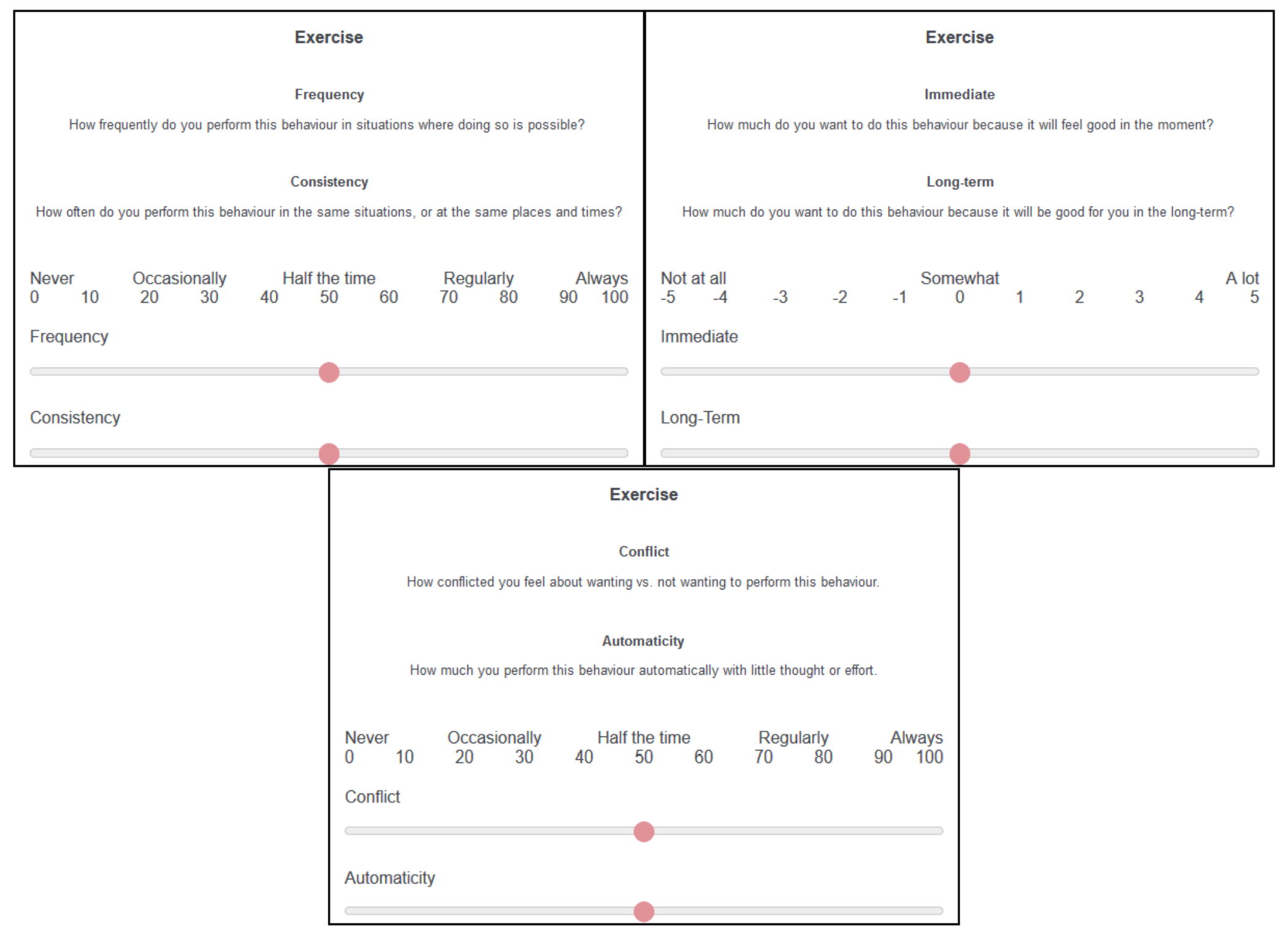


Figure SM-2. Examples of the four screens used to collect judgments in Study 2.
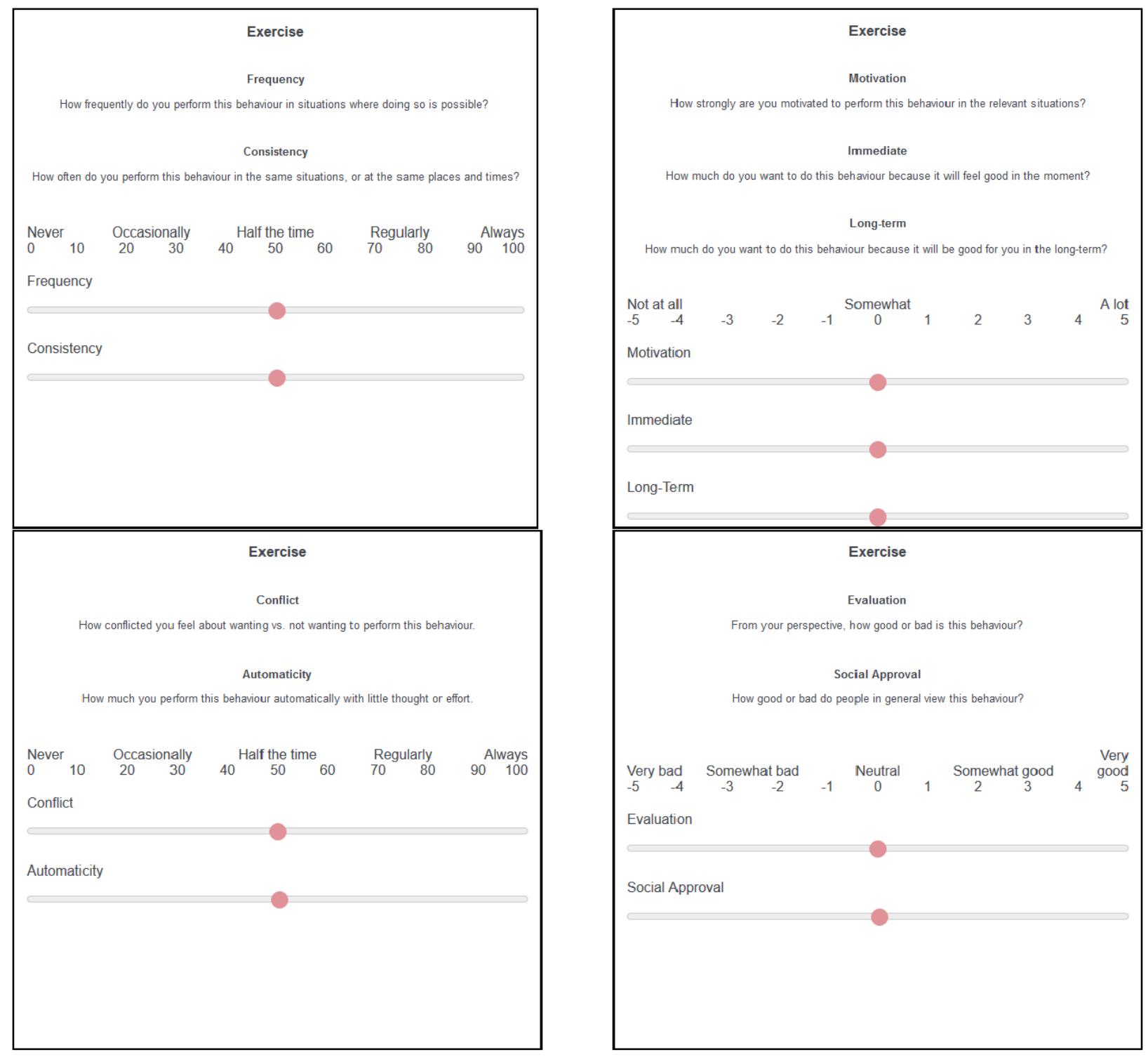
Figure SM-3. Examples of the six screens used to collect judgments in Study 3.

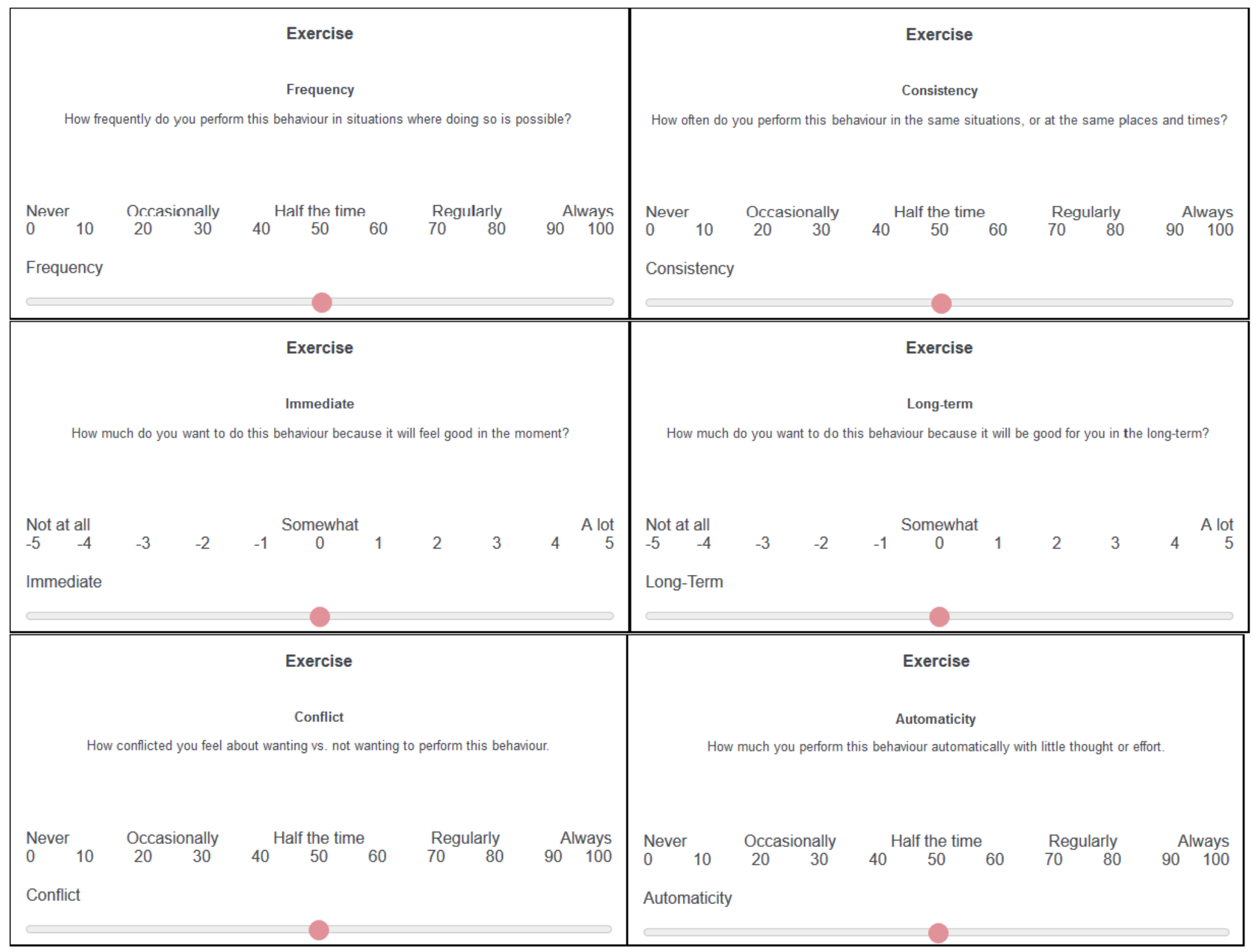




\section{Group correlation matrices}

Table SM-1 presents the correlation matrices for all three studies. As can be seen, the same general pattern of correlations emerged across studies. See the table heading and the main article for further details. 
Table SM-1. Pearson correlations between measures in Studies 1-all/2/3 (across participants and behaviors within participants).

\begin{tabular}{|c|c|c|c|c|c|c|c|c|c|c|}
\hline & Consistency & $\begin{array}{c}\text { Immediate } \\
\text { Reward }\end{array}$ & $\begin{array}{l}\text { Long-term } \\
\text { Reward }\end{array}$ & Conflict & Automaticity & Self-control & Neuroticism & $\begin{array}{c}\text { Social } \\
\text { Approval }\end{array}$ & Motivation & $\begin{array}{l}\text { Rated } \\
\text { Valence }\end{array}$ \\
\hline Regularity & $.68 / .66 / .71$ & $.47 / .50 / .39$ & $.35 / .39 / .30$ & $-.05 / .03 / .00$ & $.66 / .61 / .66$ & $-.03 /-.04 /-.01$ & $.01 / .03 /-.01$ & /.24/ & /.66/ & /.41/ \\
\hline Consistency & & $.33 / .40 / .38$ & $.24 / .29 / .27$ & $-.04 / .02 / .02$ & $.50 / .45 / .64$ & $-.03 /-.07 / .01$ & $.01 / .03 / .01$ & $/ .19 /$ & /.48/ & /.28/ \\
\hline Immediate Reward & & & $.33 / .46 / .27$ & $.01 / .06 / .07$ & $.39 / .42 / .38$ & $-.04 /-.02 / .02$ & $.01 / .03 /-.03$ & $/ .27 /$ & $/ .70 /$ & /.39/ \\
\hline Long-Term Reward & & & & $-.20 /-.06 /-.07$ & $.20 / .26 / .14$ & $.00 /-.01 / .04$ & $.00 /-.01 /-.05$ & /.61/ & $/ .50 /$ & /.68/ \\
\hline Conflict & & & & & $-.04 / .03 / .01$ & $-.11 /-.09 /-.04$ & $.08 / .12 / .05$ & $\mid-.06 /$ & $/ .05 /$ & $\mid-.06 /$ \\
\hline Automaticity & & & & & & $-.06 /-.05 /-.01$ & $.03 / .06 /-.01$ & $/ .14 /$ & $/ .53 /$ & $/ .30 /$ \\
\hline Self-control & & & & & & & $-.34 /-.48 /-.23$ & /.01/ & $\mid-.04 /$ & $\mid-.02 /$ \\
\hline Neuroticism & & & & & & & & $/-.01 /$ & $/ .04 /$ & $/ .01 /$ \\
\hline Social Approval & & & & & & & & & $/ .32 /$ & /.76/ \\
\hline Motivation & & & & & & & & & & /.48/ \\
\hline
\end{tabular}




\section{Judgment means for the 80 behaviors from Study 2}

For researchers interested in using norms established here for the 80 behaviors, Table SM-2 presents the mean standardized rating across participants for each behavior on each $\mathrm{SAM}^{2}$ judgment in Study 2. Study 2 was used because it contained the greatest number of measures (similar norms can be computed for Studies 1-all and 3 using the data files and scripts on the OSF site for this article). The means and standard deviations used to compute the standardized values are shown at the bottom, such that mean values in the original scales can be recovered. 


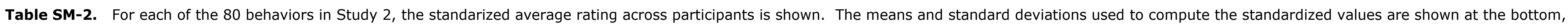
such that mean values in the original scales can be recovered. A sortable spreadsheet for this table can be found at: https://osf.io/s34bj/

Average rating

\begin{tabular}{|c|c|c|c|c|c|c|c|c|c|c|c|}
\hline Domain & Valence & Behavior & Regularity & Consistency & Motivation & Immediate & Long-Term & Conflict & Automaticity & $\begin{array}{l}\text { Rated } \\
\text { Valence }\end{array}$ & $\begin{array}{c}\text { Social } \\
\text { Approval }\end{array}$ \\
\hline \multirow[t]{8}{*}{ Food and drink } & - & Drink alcohol. & -0.97 & -0.62 & -0.50 & 0.17 & -1.16 & -0.22 & -0.95 & -0.60 & -0.11 \\
\hline & - & Eat dessert. & -0.51 & -0.40 & 0.38 & 0.83 & -0.86 & 0.82 & -0.47 & -0.21 & -0.16 \\
\hline & - & Eat fast foods. & -0.72 & -0.50 & 0.14 & 0.73 & -1.19 & 1.63 & -0.80 & -0.94 & -0.95 \\
\hline & - & Drink soft drinks. & -0.04 & 0.25 & 0.09 & 0.53 & -0.83 & 0.47 & -0.06 & -0.63 & -0.53 \\
\hline & + & Eat fruit. & 0.58 & 0.32 & 0.92 & 1.01 & 1.25 & -1.25 & 0.45 & 1.12 & 1.16 \\
\hline & + & Eat healthy snacks. & 0.08 & -0.07 & 0.52 & 0.48 & 1.19 & -0.03 & 0.06 & 0.95 & 1.06 \\
\hline & + & Eat vegetables. & 1.12 & 1.20 & 1.04 & 0.86 & 1.34 & -0.67 & 0.89 & 1.22 & 1.22 \\
\hline & + & Check food labels before making purchases. & 0.32 & 0.39 & 0.50 & 0.06 & 0.87 & -1.18 & 0.42 & 0.69 & 0.46 \\
\hline \multirow{8}{*}{ Exercise } & - & Be sedentary for long periods of time. & 0.09 & 0.33 & -0.41 & -0.32 & -1.03 & 0.89 & 0.66 & -1.09 & -1.11 \\
\hline & - & Avoid long walks. & -1.28 & -1.10 & -1.16 & -0.91 & -0.94 & -0.20 & -0.90 & -1.15 & -0.91 \\
\hline & - & Reward myself with food and/or drink after exercise. & -0.44 & -0.52 & 0.14 & 0.36 & -0.76 & 0.51 & -0.22 & -0.39 & -0.43 \\
\hline & - & Use the lift instead of taking the stairs. & -0.59 & -0.53 & -0.21 & 0.08 & -0.57 & 0.14 & -0.07 & -0.56 & -0.36 \\
\hline & + & Exercise. & -0.27 & -0.23 & 0.01 & -0.02 & 1.10 & 1.06 & -0.71 & 1.03 & 1.19 \\
\hline & + & Walk or bike when possible . & 0.35 & 0.22 & 0.54 & 0.39 & 1.06 & 0.10 & 0.23 & 0.92 & 0.95 \\
\hline & + & Participate in sports activities and clubs. & -1.48 & -1.34 & -0.88 & -0.54 & 0.51 & -0.29 & -1.71 & 0.53 & 0.95 \\
\hline & + & Take standing and walking breaks when sitting for long periods of time. & -0.35 & -0.29 & 0.03 & 0.36 & 0.82 & -0.37 & -0.51 & 0.69 & 0.60 \\
\hline \multirow[t]{8}{*}{ Affective } & - & Use substances to relax. & -1.83 & -1.91 & -1.84 & -1.53 & -1.34 & -0.94 & -1.98 & -1.07 & -1.15 \\
\hline & - & Worry. & 0.94 & 0.63 & -0.18 & -1.71 & -1.15 & 2.06 & 1.48 & -1.07 & -1.11 \\
\hline & - & Criticise myself. & 0.93 & 0.53 & 0.19 & -1.49 & -0.73 & 1.20 & 1.08 & -0.75 & -0.83 \\
\hline & - & Ignore my own needs & -0.11 & -0.16 & -0.81 & -1.54 & -1.03 & 1.44 & 0.26 & -1.21 & -1.05 \\
\hline & + & Take time to relax. & 0.45 & 0.50 & 1.25 & 1.66 & 0.77 & 0.61 & 0.38 & 0.85 & 0.67 \\
\hline & + & Do at least one thing a day that I enjoy and look forward to. & 0.38 & -0.04 & 0.95 & 1.29 & 0.82 & 0.05 & 0.01 & 0.99 & 0.94 \\
\hline & + & Express my emotions constructively. & -0.19 & -0.42 & 0.28 & 0.20 & 0.78 & 0.83 & -0.74 & 0.71 & 0.71 \\
\hline & + & View challenges with a positive attitude. & 0.41 & 0.11 & 0.57 & 0.74 & 1.06 & 0.40 & -0.23 & 0.89 & 0.96 \\
\hline \multirow[t]{8}{*}{ Social } & - & Use bad language in public. & -0.41 & -0.94 & -1.08 & -0.90 & -1.08 & 0.12 & -0.03 & -1.29 & -1.53 \\
\hline & - & Interrupt others. & -1.42 & -1.59 & -1.71 & -1.77 & -1.26 & 0.15 & -1.39 & -1.59 & -1.59 \\
\hline & - & Pay little attention to others when they are talking. & -1.31 & -1.22 & -1.43 & -1.44 & -1.14 & -0.27 & -0.75 & -1.46 & -1.42 \\
\hline & - & Make myself the centre of conversation. & -1.44 & -1.35 & -1.76 & -1.56 & -0.95 & -0.50 & -1.76 & -1.11 & -0.94 \\
\hline & + & Maintain contact with family. & 0.94 & 0.99 & 0.89 & 0.87 & 1.07 & -0.12 & 0.50 & 0.99 & 1.10 \\
\hline & + & Maintain contact with friends. & 0.46 & 0.10 & 0.91 & 1.08 & 1.08 & 0.15 & 0.17 & 0.98 & 1.19 \\
\hline & + & Hold doors open for others. & 1.48 & 1.02 & 1.41 & 1.54 & 0.57 & -1.53 & 1.96 & 1.22 & 1.11 \\
\hline & + & Say "please" and "thank you". & 2.00 & 2.09 & 1.90 & 1.87 & 1.19 & -2.06 & 2.26 & 1.40 & 1.27 \\
\hline \multirow[t]{8}{*}{ Technology } & - & Spend a large amount of time on social media. & 0.76 & 0.91 & 0.46 & 0.53 & -0.72 & 0.58 & 1.36 & -0.78 & -0.55 \\
\hline & - & Use my phone as a social crutch (e.g. use my phone when I am alone in social situ. & 1.03 & 1.09 & 0.95 & 1.08 & -0.40 & 0.21 & 1.22 & -0.45 & -0.67 \\
\hline & - & Check my phone multiple times a day. & 1.99 & 1.84 & 1.33 & 1.21 & -0.25 & -0.22 & 2.02 & -0.33 & -0.39 \\
\hline & - & Use my phone whilst on the toilet & 0.66 & 0.86 & 0.44 & 0.57 & -0.33 & -1.42 & 0.91 & -0.28 & -0.57 \\
\hline & + & Make back-up copies of important documents and files. & -0.13 & -0.08 & 0.31 & -0.35 & 1.14 & -0.92 & -0.52 & 1.05 & 0.88 \\
\hline & + & Charge my devices. & 1.65 & 2.00 & 1.55 & 1.23 & 1.03 & -1.71 & 1.97 & 0.79 & 0.61 \\
\hline & + & Limit the amount of time each day I spend using technology. & -1.93 & -1.77 & -1.34 & -1.25 & 0.26 & 0.35 & -1.41 & 0.33 & 0.33 \\
\hline & + & Restrict my use of technology before sleep. & -2.01 & -1.73 & -1.43 & -1.27 & 0.34 & 0.74 & -1.57 & 0.43 & 0.53 \\
\hline \multirow{6}{*}{ Work and study } & - & Procrastinate. & 0.79 & 0.62 & 0.48 & 0.55 & -1.18 & 2.25 & 0.87 & -1.09 & -1.14 \\
\hline & - & Work whilst watching TV or listening to music. & 0.53 & 0.49 & 0.49 & 0.67 & -0.32 & -0.08 & 0.62 & -0.10 & -0.48 \\
\hline & - & Skip lectures. & -1.87 & -1.78 & -1.43 & -0.98 & -1.50 & 0.84 & -2.03 & -1.53 & -1.43 \\
\hline & - & Multi-task during work. & 0.67 & 0.65 & 0.54 & 0.31 & 0.23 & 0.07 & 0.65 & 0.22 & 0.19 \\
\hline & + & Study for my course(s). & 0.98 & 0.93 & 0.80 & 0.14 & 1.28 & 0.81 & 0.00 & 1.30 & 1.20 \\
\hline & + & Take study breaks & 0.28 & 0.01 & 0.81 & 0.90 & 0.36 & 0.80 & -0.06 & 0.69 & 0.65 \\
\hline
\end{tabular}




\begin{tabular}{|c|c|c|c|c|c|c|c|c|c|c|}
\hline & Set goals before engaging in a task. & 0.14 & -0.13 & 0.43 & 0.39 & 1.01 & -0.39 & -0.25 & 0.76 & 0.89 \\
\hline & $+\quad$ Pack what I need the night before. & 0.35 & 0.46 & 0.38 & 0.07 & 0.95 & 0.18 & -0.11 & 0.74 & 0.56 \\
\hline \multirow[t]{8}{*}{ Personal hygiene } & Pick my nose. & -0.98 & -1.23 & -1.10 & -0.65 & -0.93 & -0.70 & -0.11 & -1.22 & -1.79 \\
\hline & Pick my spots and/ or scabs. & 0.02 & -0.02 & -0.57 & -0.20 & -1.31 & 0.59 & 0.19 & -1.22 & -1.53 \\
\hline & - $\quad$ Chew on pencils and/ or pens. & -1.75 & -1.79 & -2.13 & -1.99 & -1.39 & -1.64 & -1.36 & -1.24 & -1.29 \\
\hline & Bite my nails. & -1.10 & -1.47 & -1.56 & -1.50 & -1.41 & -0.62 & -0.72 & -1.40 & -1.48 \\
\hline & Shower every day. & 1.03 & 1.47 & 1.12 & 1.47 & 1.08 & -1.26 & 1.21 & 1.10 & 1.17 \\
\hline & Cover my mouth when sneezing, coughing or yawning. & 1.66 & 1.55 & 1.63 & 1.29 & 0.88 & -1.74 & 1.85 & 1.26 & 1.18 \\
\hline & Brush my teeth twice a day. & 0.66 & 1.05 & 0.79 & 0.80 & 1.27 & -0.29 & 0.57 & 1.23 & 1.30 \\
\hline & Go to sleep and wake up at the same times. & -0.37 & -0.18 & -0.05 & -0.19 & 0.80 & 0.61 & -0.49 & 0.56 & 0.64 \\
\hline \multirow[t]{8}{*}{ Household } & Allow messes to build up in my work area. & -0.43 & -0.32 & -1.32 & -1.29 & -1.16 & 0.35 & 0.10 & -1.19 & -1.28 \\
\hline & Ignore stains and spills. & -1.63 & -1.26 & -1.45 & -1.38 & -1.13 & 0.10 & -1.28 & -1.36 & -1.48 \\
\hline & Leave dishes to wash later & 0.24 & 0.44 & -0.19 & 0.06 & -1.07 & 1.24 & 0.21 & -0.97 & -0.97 \\
\hline & Leave clothes lying around. & 0.04 & 0.43 & -0.61 & -0.42 & -1.22 & 0.08 & 0.40 & -1.10 & -1.24 \\
\hline & Wash my clothes. & 1.19 & 1.40 & 0.97 & 0.69 & 1.18 & -0.50 & 0.72 & 1.22 & 1.17 \\
\hline & Put things back after I have finished using them. & 0.74 & 0.63 & 0.55 & 0.09 & 1.02 & -0.13 & 0.62 & 0.87 & 0.79 \\
\hline & Empty the bins. & 0.50 & 0.66 & -0.05 & 0.05 & 0.94 & 0.35 & 0.23 & 0.96 & 0.96 \\
\hline & Clean my residence & 0.78 & 0.90 & 0.46 & 0.70 & 1.17 & 0.70 & 0.16 & 1.10 & 1.08 \\
\hline \multirow[t]{8}{*}{ Finance } & Dip into funds I have set aside. & -0.58 & -0.89 & -0.74 & -0.22 & -1.18 & 2.40 & -1.29 & -1.31 & -0.98 \\
\hline & Spend to make myself feel better. & -0.39 & -0.70 & 0.11 & 0.70 & -0.86 & 1.74 & -0.47 & -0.53 & -0.20 \\
\hline & Buy brand name products. & 0.06 & -0.04 & 0.02 & 0.05 & -0.29 & 0.05 & -0.14 & -0.13 & 0.27 \\
\hline & Make impulsive purchases & -0.45 & -0.96 & -0.12 & 0.54 & -1.10 & 2.11 & -0.40 & -0.89 & -0.67 \\
\hline & Budget. & 0.50 & 0.35 & 0.69 & 0.05 & 1.24 & 0.20 & -0.25 & 0.91 & 0.80 \\
\hline & Buy from charity and/ or second-hand shops. & -1.20 & -1.23 & -0.48 & -0.43 & 0.18 & -1.22 & -1.35 & 0.62 & 0.39 \\
\hline & Use shopping lists & -0.20 & 0.21 & 0.15 & 0.34 & 0.55 & -1.14 & -0.33 & 0.58 & 0.48 \\
\hline & Shop for groceries. & 1.01 & 1.10 & 0.77 & 0.63 & 0.93 & -0.56 & 0.48 & 0.81 & 0.74 \\
\hline \multirow[t]{10}{*}{ Environment } & Litter. & -2.58 & -2.58 & -2.90 & -2.79 & -1.58 & -1.09 & -2.43 & -1.97 & -1.81 \\
\hline & Buy new condition items & 0.52 & 0.17 & 0.52 & 0.69 & 0.36 & 0.22 & 0.19 & 0.18 & 0.42 \\
\hline & Leave plug sockets switched on & -0.21 & 0.13 & -1.18 & -1.50 & -0.99 & -0.90 & 0.04 & -1.19 & -1.06 \\
\hline & Throw away food. & -0.96 & -0.57 & -1.64 & -1.78 & -1.16 & 1.20 & -1.06 & -1.46 & -1.39 \\
\hline & Turn off lights when leaving a room. & 0.76 & 0.81 & 0.77 & 0.17 & 0.99 & -1.69 & 1.25 & 0.91 & 0.83 \\
\hline & Recycle. & 1.05 & 1.11 & 1.04 & 0.47 & 1.21 & -1.13 & 0.97 & 1.23 & 1.21 \\
\hline & Reuse carrier bags. & 1.02 & 0.98 & 1.02 & 0.61 & 1.02 & -1.26 & 0.92 & 1.07 & 1.00 \\
\hline & $+\quad$ Use reusable cups. & 0.02 & 0.00 & 0.05 & -0.24 & 0.55 & -1.19 & 0.35 & 0.75 & 0.76 \\
\hline & Grand Mean & 54.81 & 59.83 & 0.86 & 1.09 & 0.52 & 40.77 & 55.56 & 0.88 & 0.99 \\
\hline & SD & 17.00 & 12.39 & 1.45 & 1.36 & 2.53 & 9.28 & 14.05 & 2.34 & 2.43 \\
\hline
\end{tabular}




\section{Regression analysis pipeline}

The regression analysis pipeline described here was used in all regression analyses to follow. All later tables of regression results were produced using this pipeline.

The primary goals of our analysis pipeline were to: (1) identify effects, (2) establish their effect sizes, and (3) assess their generalizability across participants and behaviors. The dependent variable (behaviour regularity) and its predictors (e.g., consistency immediate reward, long-term reward, conflict, automaticity) were all standardized so that we could specify each predictor's effect in standard deviation units. Thus, each estimated regression coefficient provides a measure of effect size, indicating the standard-deviation-unit change in the dependent variable associated with each standarddeviation-unit change in the predictor. The sign of these standardized coefficients further indicates the direction of the relationship. If, for example, a standardized coefficient for the relation between automaticity and behaviour regularity happened to be .60 , this meant that behaviour regularity increased positively by .60 of a standard deviation for each standard deviation increase in automaticity. The larger the absolute value of a coefficient, the larger its effect size.

For each regression analysis, we implemented a sequence of three multilevel mixed-effect models (using the lme4 package in R; Bates, Mächler, Bolker, \& Walker, 2015). We will refer these models as Model 1, Model 2, and Model 3. These models were multilevel because they predicted a dependent variable such as behaviour regularity using both behavior-level predictors (consistency, immediate reward, long-term reward, conflict, automaticity) and individual-level predictors (self-control, neuroticism). These models were mixed effect because they simultaneously assessed both fixed effects (predictors at the individual and behavior levels) and random effects (random intercepts and slopes capturing variability of effects at the individual and behavior levels). Assessing random effects is pivotal for generalizing results beyond a current sample of participants and behaviors (Barr, Levy, Scheepers, \& Tily, 2013). Multilevel mixed-effect modelling offers a powerful approach for establishing generalizability across participants and behaviors simultaneously.

In the first stage of our analysis pipeline, Model 1 identified predictors likely to have meaningful effects on the dependent variable (both main effects and interactions). Model 1 included all predictors of interest at the behavior and individual levels, all interactions up through three-way, and random intercepts for behaviors and participants. This relatively liberal model served to identify potentially important predictors that were subsequently examined more closely and conservatively in Models 2 and 3. For a predictor to pass this initial screening, the $t$ for its estimated regression coefficient had to be greater than $|1.96|$ (associated with a $p$-value $\leq .05$ ). We assumed that any effect that failed this initial screening would be unlikely to have a meaningful impact on the dependent variable.

For each potentially important predictor identified in Model 1, we then assessed it more conservatively in a unique Model 2 that tested it maximally (Barr et al., 2013). Specifically, maximal testing established whether a predictor's effect in Model 1 generalized beyond participant-level and behavior-level variability for the effect in the underlying population of possible observations. Imagine, for example, that the .60 estimated regression coefficient for automaticity survived initial screening in Model 1. If large individual differences in participants and behaviors are present, then the observed effect may not generalize to the broader populations of participants and behaviors. To test an observed effect in Model 1 maximally, Model 2 included one empirically-determined random slope for each participant that modeled the effect for that participant. Additionally, Model 2 included one empirically-determined random slope for each behavior that modeled the effect for that behavior. Of interest was whether the $t$ for the fixed effect in Model 2 remained greater than |1.96| once the variances of the random effects for participants and behaviors were accounted for simultaneously. If the fixed effect passed this maximal testing, we concluded that it generalizes beyond the current samples of participants and behaviors. If the effect failed maximal testing, we assumed that it does not generalize and can be explained in terms of individual-level and behavior-level variability. 
Unfortunately, including appropriate random slopes simultaneously in Model 2 for each and every predictor that survives initial screening in Model 1 is typically not possible, as the sheer complexity of the model disrupts optimization and convergence. To circumvent this problem, Barr et al. (2013, p. 276) suggested maximally testing each effect of interest one at a time (i.e., including appropriate random slopes for participants and behaviors associated with the fixed effect of interest, while not including random slopes for any remaining fixed effects). Thus, when maximally testing the effect of (say) automaticity, a unique Model 2 was constructed by adding random slopes for automaticity to Model 1 but not adding random slopes for any other fixed effect. In this manner, a unique Model 2 was constructed for each fixed effect that passed Model 1 screening. Importantly, whenever a higherorder interaction passed Model 1 screening, random slopes were also included for all lower-order interactions and main effect terms nested within it (see Barr et al., 2013).

If a predictor passed maximal testing in Model 2, it was evaluated one more time in a unique Model 3 that established how much unique variance in Model 2 was associated with it. In each Model 3, we dropped the main effect or interaction being tested from its Model 2, along with any interactions containing it and any associated random slopes, while keeping everything else the same as in Model 2. We then subtracted the total variance for the predictor's Model 3 from the total variance for its Model 2. The difference in $R^{2}\left(\Delta R^{2}\right.$ expressed as a percentage) established how much unique variance the predictor captured when included as a fixed effect together with associated random effects in Model 2.

Using this analysis pipeline, we established predictors associated with effects that generalize across participants and behaviors (i.e., predictors that survived maximal testing in Model 2). For each predictor that generalized, we obtained two measures of its effect size: (1) its standardized regression coefficient in Model 2, and (2) its $\Delta R^{2}$ derived from Model 3. 


\section{Assessing the validity of the a priori valence assignments}

Participants in Study 2 rated the valence of each behavior in Table 1. For each behavior participants were asked to judge, "From your perspective, how good or bad is the behaviour?", using a scale from -5 to 5, with the labels: Very bad, Somewhat bad, Neutral, Somewhat good, Very good. The intraclass correlation for inter-rater agreement was .55 (ICC2).

We predicted that rated evaluations of behavior valence in Study 2 would confirm our a priori assignments of positive versus negative behavior valence in Table 1. The point-biserial correlation between these two judgments, .68, supports this prediction. Figure SM-4 plots the average rated valence of the a priori positive and negative behaviors in the original scale units $(-5$ to +5$)$. As can be seen, the a priori positive and negative behaviors did not overlap in rated valence, confirming our original valence assignments.

To assess the predictors of a priori and rated valence, we applied our standard regression analysis pipeline, except that logistic regression was performed for the binary dependent variable of a priori valence. Table SM-3 presents the main effects from these two analyses. As the top half of Table SM-3 illustrates, approximately $100 \%$ of the variance in our a priori valence assignments was explained by long-term reward and social approval (because long-term reward and social approval correlated .61, removing either one from their respective Model 2 produced a $-1 \%$ value in $\Delta R^{2}$ ). These results suggest that long-term reward and social approval drove our a priori judgments of valence.

Interestingly, prediction of rated valence reflected many more factors (Table SM-3, bottom half). When participants rated valence themselves, social approval became by far the most important predictor. Long-term reward was the second strongest predictor but was much weaker than social approval. Interestingly, behavior regularity was the third most important predictor, indicating that as participants performed a behavior more regularly, they viewed it as increasingly positive. Similarly, automaticity and immediate reward also explained significant positive variance in rated valence. The importance of regularity, automaticity, and immediate reward suggest that behavior strength and enjoyment induce positive attributions of behaviors. 


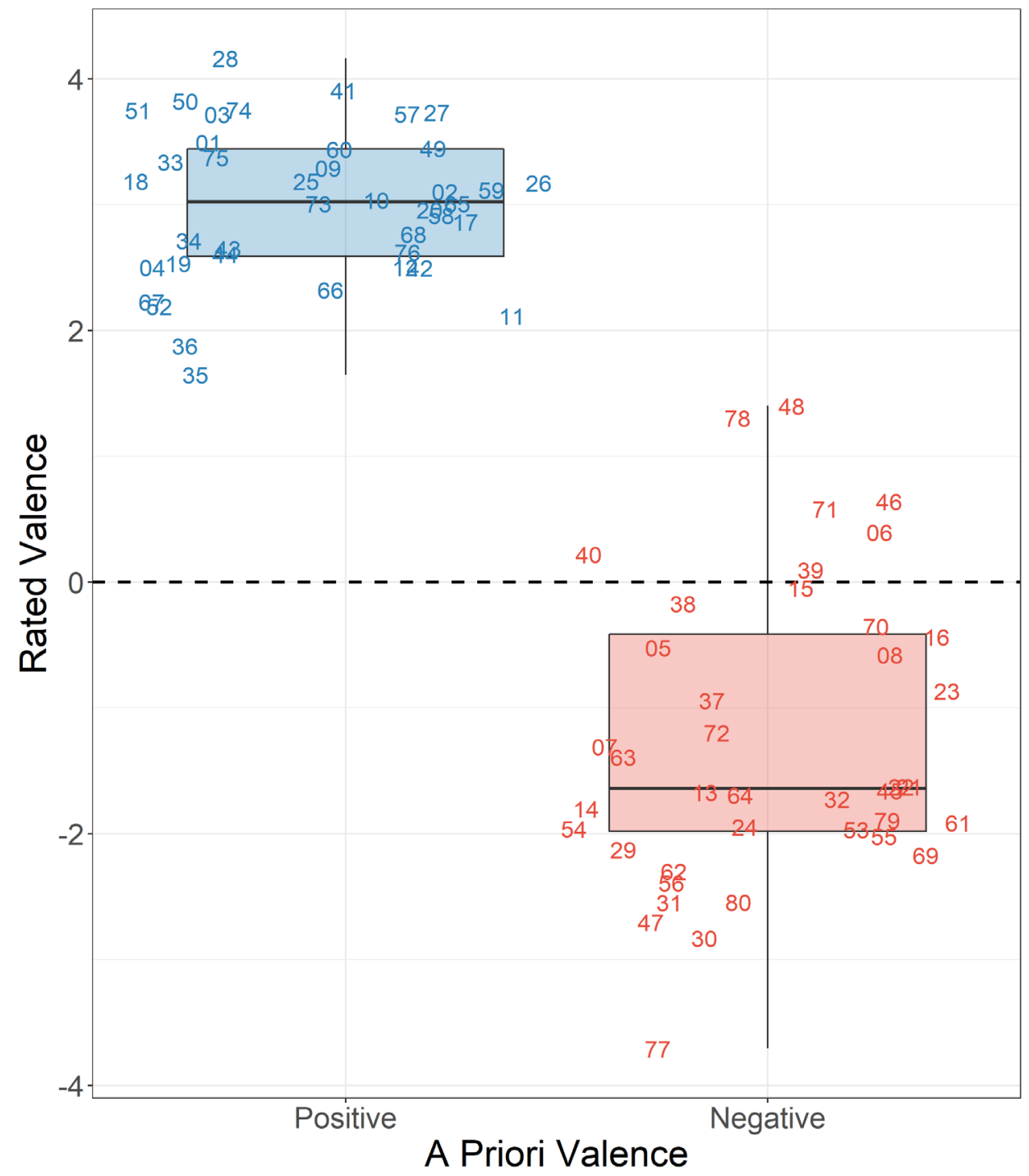

Figure 4. Mean rated valence of the 80 behaviors for habits assigned to a priori positive and negative groups in Table 1 (Study 2). Mean valence is shown in the original scale units $(-5$ to +5$)$. The numeric identifiers for specific behaviors map onto to the numbers in Table 1, such that specific behaviors in Table 1 can be identified here. 
Table SM-3. Results from the group regressions in Study 2 for the prediction of a priori valence (top) and rated valence (bottom) at the behavior level, including predictors for regularity, consistency, immediate reward, long-term reward, conflict, automaticity, and social approval. Section 4 of the SM describes the analysis pipeline used to produce Models 1, 2, and 3.

\begin{tabular}{|c|c|c|c|c|c|c|c|c|c|c|}
\hline \multirow[b]{2}{*}{ DV / Predictor } & \multicolumn{3}{|c|}{ Model 1} & \multicolumn{5}{|c|}{ Model 2} & \multicolumn{2}{|c|}{ Model 3} \\
\hline & Estimate & $\mathbf{S E}$ & $t$ & Estimate & SE & $t$ & $R^{2}$ & AIC & $\overline{\Delta R^{2}}$ & $\Delta \mathbf{A I C}$ \\
\hline \multicolumn{11}{|l|}{ DV: A priori valence } \\
\hline Regularity & .10 & .269 & 0.35 & & & & & & & \\
\hline Consistency & -.05 & .230 & -0.22 & & & & & & & \\
\hline Immediate Reward & -.27 & .232 & -1.15 & & & & & & & \\
\hline Long-term Reward & .89 & .235 & 3.79 & .89 & .235 & 3.79 & 100 & 228 & -1 & -7 \\
\hline Conflict & -.16 & .174 & -0.90 & & & & & & & \\
\hline Automaticity & -.10 & .213 & -0.47 & & & & & & & \\
\hline Social Approval & 1.06 & .214 & 4.94 & 1.06 & .214 & 4.94 & 99 & 228 & -1 & -5 \\
\hline \multicolumn{11}{|l|}{ DV: Rated valence } \\
\hline Regularity & .13 & .007 & 18.14 & .13 & .016 & 8.24 & 67 & 24609 & -3 & 841 \\
\hline Consistency & -.02 & .006 & -2.87 & -.02 & .011 & -2.15 & 66 & 24956 & -1 & 178 \\
\hline Immediate Reward & .05 & .006 & 7.91 & .04 & .012 & 3.47 & 67 & 24727 & -2 & 461 \\
\hline Long-term Reward & .19 & .007 & 27.95 & .18 & .016 & 11.34 & 68 & 24009 & -5 & 1867 \\
\hline Conflict & -.01 & .005 & -2.76 & -.01 & .008 & -1.67 & 66 & 24967 & & \\
\hline Automaticity & .09 & .006 & 15.46 & .09 & .014 & 6.41 & 67 & 24695 & -2 & 667 \\
\hline Social Approval & .43 & .007 & 64.53 & .44 & .023 & 19.02 & 70 & 23427 & -13 & 5310 \\
\hline
\end{tabular}

Note. All regressions were performed on standardized measures. Thus, an Estimate is the estimate of a standardized regression coefficient in the respective model, with SE and $t$, being the standard error and $t$ value of the estimate. $R^{2}$ is the total variance explained by Model 2 , and $\Delta R^{2}$ is the amount of variance explained by the main effect or interaction dropped in Model 3 (both in percentage points). AIC is the value of the Akaike Information Criterion for Model 2, and $\Delta$ AIC is its change for Model 3. 


\section{Clustered heatmaps for individual $\times$ behavior interactions}

A clustered heatmap of the individual $\times$ behavior interaction was presented for Study 2 in the main text (Figure 3). Figures SM-7 and SM-8 next present analogous clustered heatmaps for the individual $\times$ behavior interactions in Studies 1-all and 3, respectively. See the figure captions and the main article for further details. 


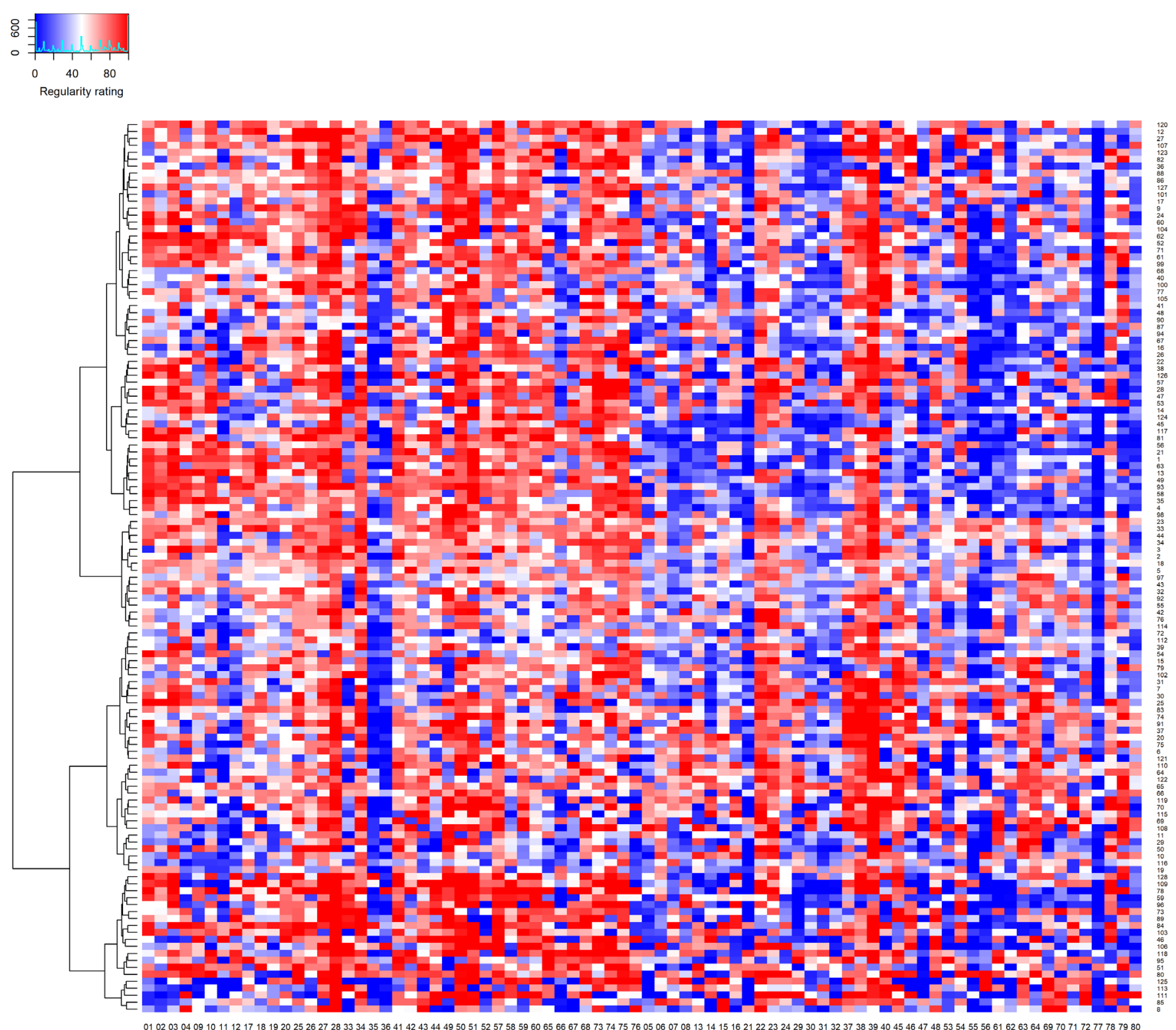

Figure SM-5. A heatmap that visualizes the individual $\times$ behavior interaction for behavior regularity in Study 1 -all. The 80 regularity judgments for each of the 128 participants are presented in a single row, with their judgments for positive behaviors in the left half, and their judgments for negative behaviors in the right half. The number below each column corresponds to the number of the corresponding behavior in Table 1 . As a cell becomes increasingly red, the regularity judgment increasingly approached 100 (on a scale of 0 to 100). As a cell becomes increasingly blue, the regularity judgment increasingly approached 0 . As a cell becomes increasingly white, the regularity judgment was increasingly approached 50 . On the left, a hierarchical clustering dendrogram establishes participants having similar vectors of regularity values across situations (from hierarchical clustering with the Ward D measure). Table 1 in the main text provides intraclass correlations that assess inter-rater reliability of the judgments in this map. 


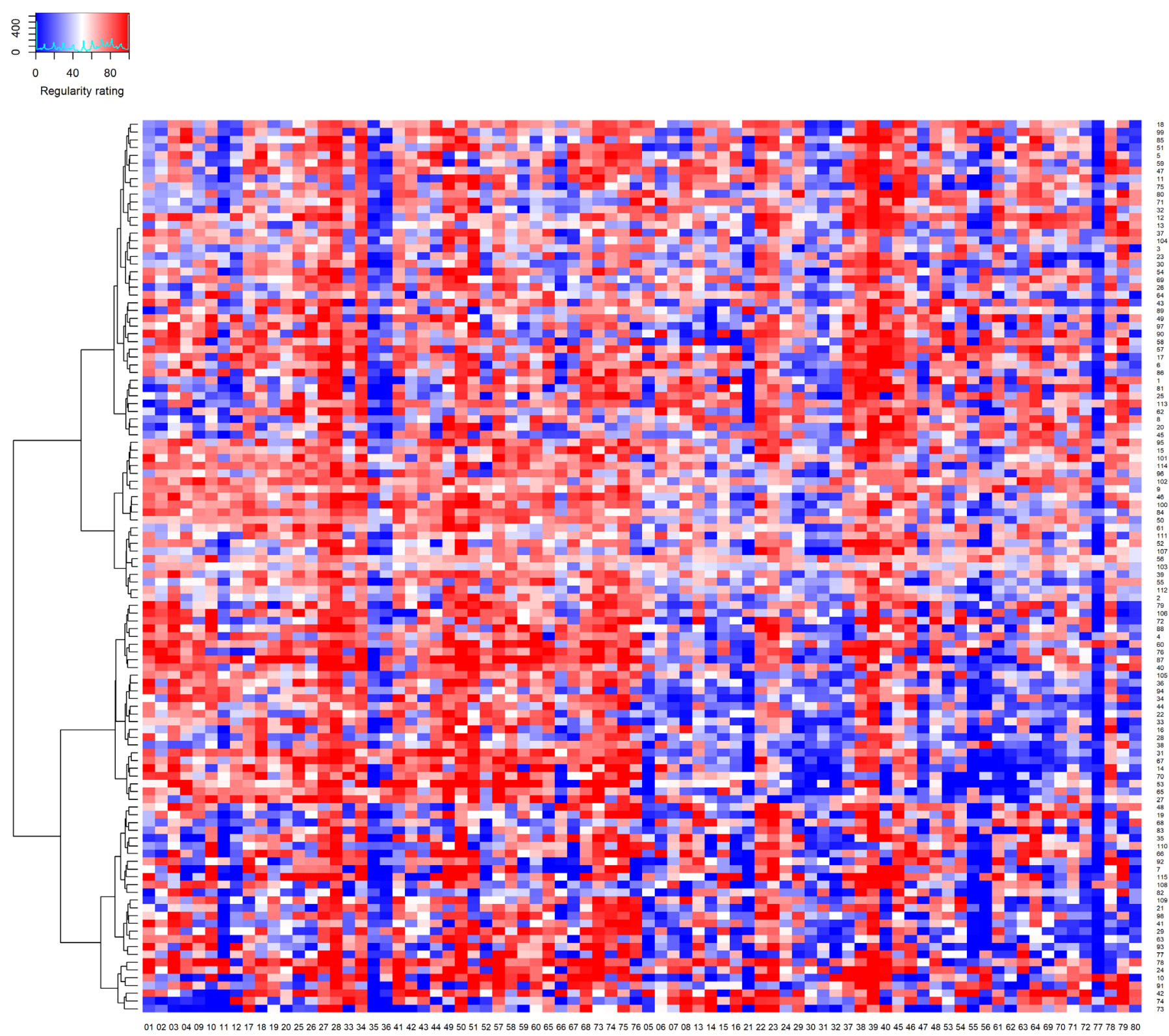

Figure SM-6. A heatmap that visualizes the individual $\times$ behavior interaction for behavior regularity in Study 3 . The 80 regularity judgments for each of the 115 participants are presented in a single row, with their judgments for positive behaviors in the left half, and their judgments for negative behaviors in the right half. The number below each column corresponds to the number of the corresponding behavior in Table 1. As a cell becomes increasingly red, the regularity judgment increasingly approached 100 (on a scale of 0 to 100). As a cell becomes increasingly blue, the regularity judgment increasingly approached 0 . As a cell becomes increasingly white, the regularity judgment was increasingly approached 50 . On the left, a hierarchical clustering dendrogram establishes groups of participants having similar vectors of regularity values across situations (from hierarchical clustering with the Ward D measure). Table 1 in the main text provides intraclass correlations that assess inter-rater reliability of the judgments in this map. 
7. Regression results for the group-level analyses of Studies 1-all, 2, and 3

The following three tables contain the complete regression results for group-level results presented in the main text.

Table SM-4 presents the regression results for Study 1-all. Table SM-5 presents the regression results for Study 2. Table SM-6 presents the regression results for Study 3.

Section 4 of the SM describes the analysis pipeline used to produce Models 1, 2, and 3 for all three studies. 
Table SM-4. Complete results from the group regressions in Study 1-all for the prediction of behavior regularity, including predictors for valence, consistency, immediate reward, long-term reward, conflict, automaticity, self-control, and neuroticism. Results for all main effects are shown, along with two- and three-way interactions in Model 1 where $t>|1.96|$. Section 4 of the SM describes the analysis pipeline used to produce

Models 1, 2, and 3 .

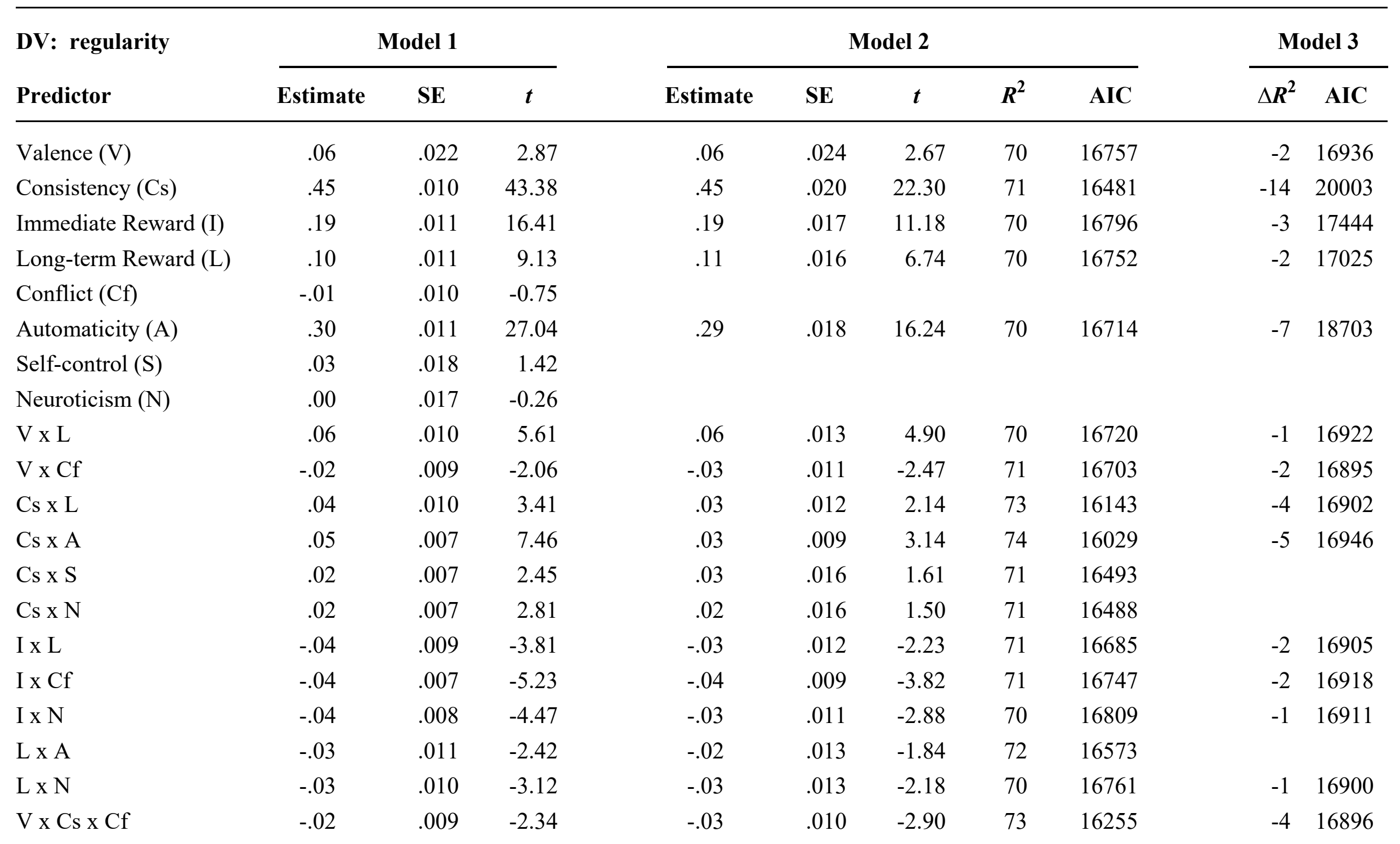




\begin{tabular}{|c|c|c|c|c|c|c|c|c|c|c|}
\hline$V \times C s \times A$ & -.02 & .009 & -2.06 & -.01 & .010 & -1.12 & 75 & 15854 & & \\
\hline V x I x L & -.03 & .009 & -2.87 & -.01 & .010 & -.92 & 71 & 16676 & & \\
\hline$V \times L \times S$ & -.02 & .010 & -1.98 & -.02 & .010 & -2.20 & 70 & 16726 & -1 & 16895 \\
\hline Cs $\times I \times A$ & -.04 & .006 & -6.10 & -.02 & .009 & -2.37 & 76 & 15834 & -7 & 16928 \\
\hline Cs $x \mathrm{I} \times \mathrm{S}$ & .03 & .007 & 4.51 & .01 & .008 & 1.45 & 73 & 16290 & & \\
\hline Cs $x$ L x Cf & .02 & .009 & 2.47 & .02 & .010 & 2.29 & 73 & 16209 & -4 & 16897 \\
\hline Cs $x$ Cf $x A$ & .02 & .006 & 3.87 & .02 & .009 & 2.06 & 75 & 16003 & -6 & 16906 \\
\hline Cs $x \mathrm{~A} \times \mathrm{N}$ & .02 & .007 & 3.45 & .01 & .008 & 1.75 & 74 & 16040 & & \\
\hline Cs $x \mathrm{~S} \times \mathrm{N}$ & .02 & .008 & 2.96 & .02 & .016 & 1.39 & 71 & 16492 & & \\
\hline I x Cf x A & -.02 & .006 & -3.42 & -.03 & .007 & -3.74 & 72 & 16586 & -3 & 16902 \\
\hline I x Cfx S & -.02 & .006 & -2.61 & -.01 & .008 & -1.69 & 71 & 16760 & & \\
\hline $\mathrm{I} \times \mathrm{A} \times \mathrm{N}$ & -.03 & .008 & -3.22 & -.02 & .009 & -2.35 & 72 & 16613 & -3 & 16901 \\
\hline Cf $x A \times S$ & .02 & .007 & 2.59 & .02 & .007 & 2.10 & 71 & 16703 & -2 & 16897 \\
\hline
\end{tabular}

Note. All regressions were performed on standardized measures. Thus, an Estimate is the estimate of a standardized regression coefficient in the respective model, with SE and $t$, being the standard error and $t$ value of the estimate. $R^{2}$ is the total variance explained by Model 2, and $\Delta R^{2}$ is the amount of variance explained by the main effect or interaction dropped in Model 3 (both in percentage points). AIC is the value of the Akaike Information Criterion for Models 2 and 3. 
Table SM-5. Complete results in Study 2 from the replication of the group regressions in Study 1 for the prediction of behavior regularity, including predictors for a priori valence, consistency, immediate reward, long-term reward, conflict, automaticity, self-control, and neuroticism. Results for all main effects are shown, along with two- and three-way interactions in Model 1 where $t>|1.96|$. Section 4 of the SM describes the analysis pipeline used to produce Models 1, 2, and 3.

\begin{tabular}{|c|c|c|c|c|c|c|c|c|c|c|}
\hline $\begin{array}{l}\text { DV: regularity } \\
\text { Predictor }\end{array}$ & Estimate & SE & $t$ & Estimate & SE & $t$ & $R^{2}$ & AIC & $\Delta R^{2}$ & AIC \\
\hline Consistency (Cs) & .44 & .008 & 52.97 & .45 & .02 & 22.14 & 69 & 26809 & -10 & 30274 \\
\hline Immediate Reward (I) & .12 & .009 & 13.22 & .12 & .015 & 8.02 & 67 & 27497 & -2 & 27878 \\
\hline Conflict (Cf) & .01 & .008 & 1.24 & & & & & & & \\
\hline Automaticity (A) & .30 & .008 & 35.35 & .29 & .016 & 18.60 & 67 & 27418 & -4 & 28 \\
\hline Self-control (S) & .01 & .017 & 0.33 & & & & & & & \\
\hline Neuroticism (N) & -.01 & .017 & -0.85 & & & & & & & \\
\hline $\mathrm{V} \times \mathrm{Cf}$ & -.02 & .007 & -2.40 & -.02 & .009 & -1.83 & 68 & 27363 & & \\
\hline Cs $x$ A & .04 & .006 & 7.75 & .01 & .011 & 0.54 & 72 & 25649 & & \\
\hline Cs $x \mathrm{~S}$ & .03 & .006 & 5.12 & .03 & .015 & 2.29 & 69 & 26797 & -3 & 27730 \\
\hline I x L & -.02 & .007 & -2.46 & -.02 & .01 & -1.97 & 68 & 27319 & -2 & 27710 \\
\hline I x A & -.02 & .006 & -3.36 & -.02 & .009 & -2.49 & 68 & 27133 & -2 & 27715 \\
\hline I x N & -.02 & .007 & -2.82 & -.02 & .01 & -1.96 & 67 & 27514 & & \\
\hline $\mathrm{L} \times \mathrm{Cf}$ & -.03 & .007 & -4.58 & -.03 & .01 & -3.21 & 68 & 27323 & -2 & 27725 \\
\hline $\mathrm{L} \times \mathrm{A}$ & -.05 & .008 & -5.65 & -.03 & .012 & -2.77 & 69 & 27085 & -3 & 27736 \\
\hline
\end{tabular}




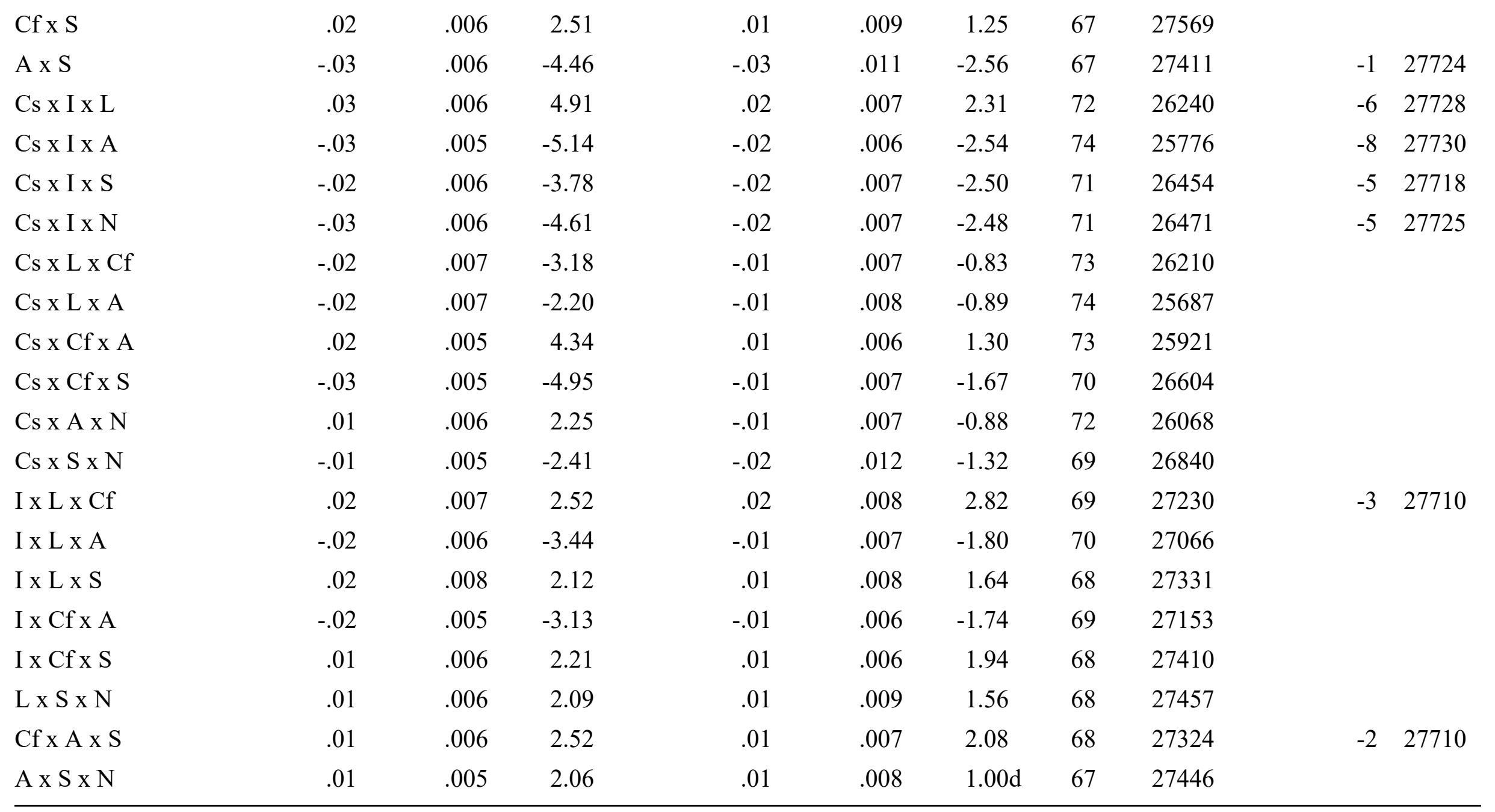

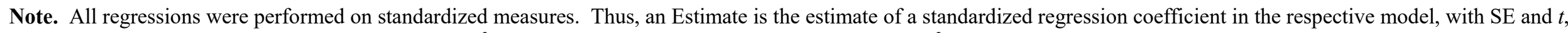
being the standard error and $t$ value of the estimate. $R^{2}$ is the total variance explained by Model 2 , and $\Delta R^{2}$ is the amount of variance explained by the main effect or interaction dropped in Model 3 (both in percentage points). AIC is the value of the Akaike Information Criterion for Models 2 and 3. 
Table SM-6. Complete results from the group regressions in Study 3 for the prediction of behavior regularity, including predictors for valence, consistency, immediate reward, long-term reward, conflict, automaticity, self-control, and neuroticism. Results for all main effects are shown, along with two- and three-way interactions in Model 1 where $t>|1.96|$. Section 4 of the SM describes the analysis pipeline used to produce

Models 1, 2, and 3 .

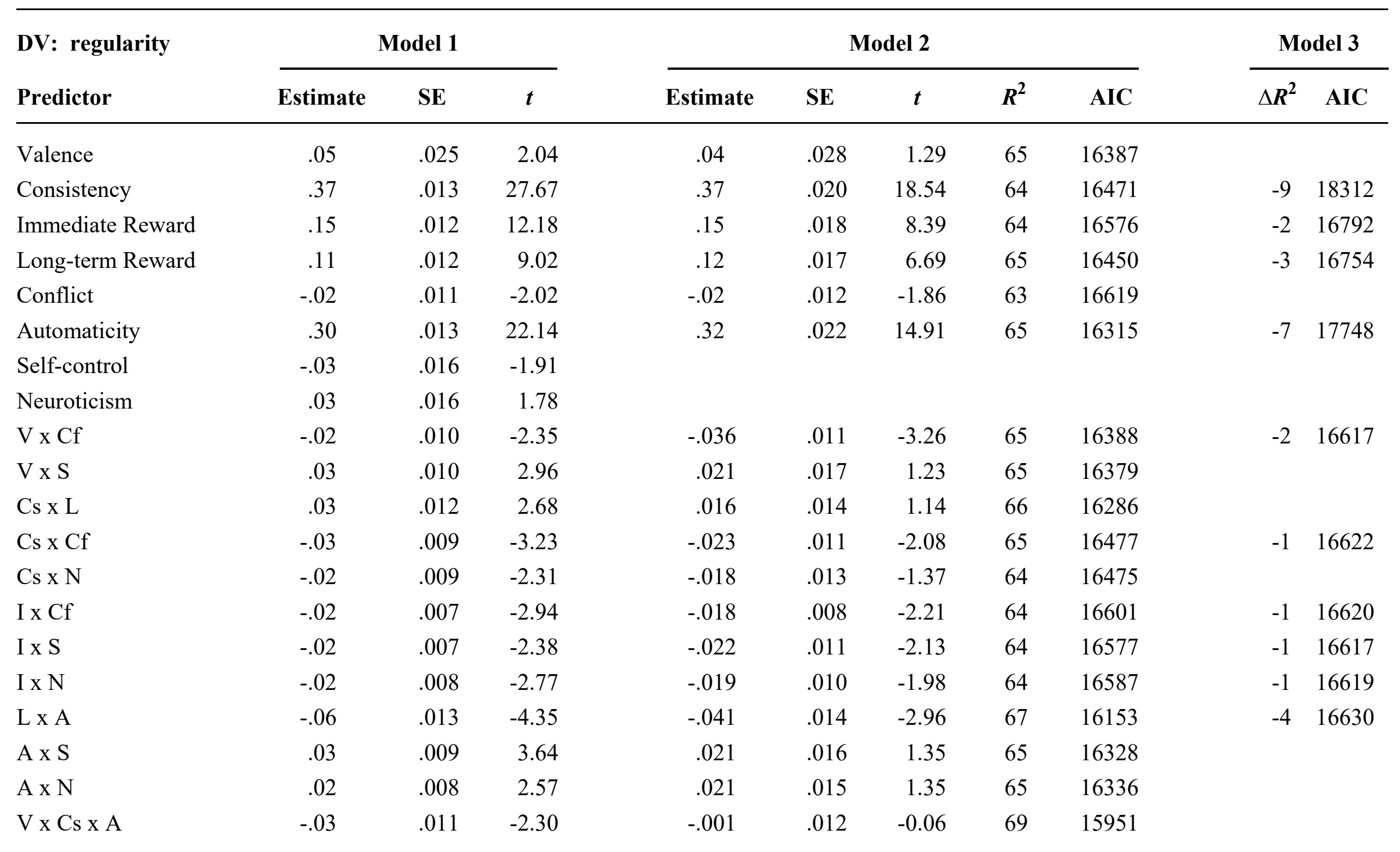




\begin{tabular}{|c|c|c|c|c|c|c|c|c|c|c|}
\hline V x I x L & -.04 & .010 & -3.65 & -.031 & .011 & -2.94 & 66 & 16361 & -2 & 16625 \\
\hline $\mathrm{V} \times \mathrm{I} \times \mathrm{N}$ & .03 & .011 & 2.84 & .018 & .011 & 1.63 & 65 & 16373 & & \\
\hline $\mathrm{Vx} \mathrm{L} \times \mathrm{Cf}$ & .03 & .010 & 3.16 & .029 & .010 & 2.90 & 65 & 16386 & -2 & 16621 \\
\hline $\mathrm{V} \times \mathrm{L} \times \mathrm{A}$ & .03 & .013 & 2.28 & .022 & .013 & 1.65 & 67 & 16120 & & \\
\hline$V \times L \times N$ & -.04 & .010 & -3.95 & -.030 & .011 & -2.84 & 65 & 16375 & -2 & 16627 \\
\hline$V \times A \times N$ & -.03 & .012 & -2.14 & -.021 & .012 & -1.71 & 66 & 16132 & & \\
\hline Cs x I x Cf & .02 & .008 & 2.25 & .019 & .008 & 2.40 & 65 & 16460 & -2 & 16616 \\
\hline Cs $\times I \times A$ & -.04 & .007 & -5.79 & -.026 & .007 & -3.73 & 68 & 16127 & -5 & 16645 \\
\hline Cs $x$ L x Cf & -.03 & .011 & -2.47 & -.015 & .012 & -1.32 & 67 & 16303 & & \\
\hline Cs x L x A & -.03 & .010 & -2.47 & -.021 & .010 & -2.02 & 69 & 15991 & -6 & 16617 \\
\hline Cs $x$ Cf $x N$ & .03 & .008 & 3.29 & .023 & .008 & 2.77 & 65 & 16490 & -2 & 16622 \\
\hline Ix Cf x A & -.02 & .007 & -1.00 & -.006 & .008 & -0.76 & 66 & 16311 & & \\
\hline $\mathrm{L} \times \mathrm{Cf} \times \mathrm{A}$ & .03 & .011 & 2.79 & .028 & .012 & 2.47 & 66 & 16193 & -3 & 16619 \\
\hline $\mathrm{L} \times \mathrm{Cf} \times \mathrm{S}$ & .02 & .009 & 2.25 & .025 & .010 & 2.65 & 65 & 16462 & -2 & 16616 \\
\hline $\mathrm{Cf} \times \mathrm{A} \times \mathrm{N}$ & -.02 & .008 & -2.78 & -.017 & .009 & -2.01 & 65 & 16329 & -2 & 16619 \\
\hline$A \times S \times N$ & .02 & .009 & 2.05 & .020 & .016 & 1.28 & 65 & 16328 & & \\
\hline
\end{tabular}

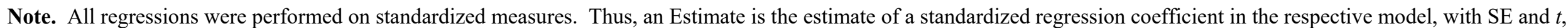
being the standard error and $t$ value of the estimate. $R^{2}$ is the total variance explained by Model 2 , and $\Delta R^{2}$ is the amount of variance explained by the main effect or interaction dropped in Model 3 (both in percentage points). AIC is the value of the Akaike Information Criterion for Models 2 and 3. 


\section{Clustered heatmaps of individual predictive correlations}

A clustered heatmap of individual predictive correlations was presented for Study 2 in the main text (Figure 6). Figures SM-7 and SM-8 present analogous clustered heatmaps for individual predictive correlations in Studies 1-all and 3, respectively. See the figure captions and the main article for further details. 
Figure SM-7. A heatmap that visualizes correlations between behavior regularity and individual factors from the Situated Action Cycle (Conflict, Immediate Reward, Long-Term Reward, Automaticity, and Consistency). The six correlations for each of the 128 participants in Study 1-all appear in a single row. As a cell becomes increasingly red, the correlation was increasingly positive. As a cell becomes increasingly blue, the correlation was increasingly negative. As a cell becomes increasingly white, the correlation increasingly approached 0 . On the left, a hierarchical clustering dendrogram establishes groups of participants having similar prediction vectors (from hierarchical clustering with the Ward D measure). Table 4 in the main text summarizes the correlations shown below.

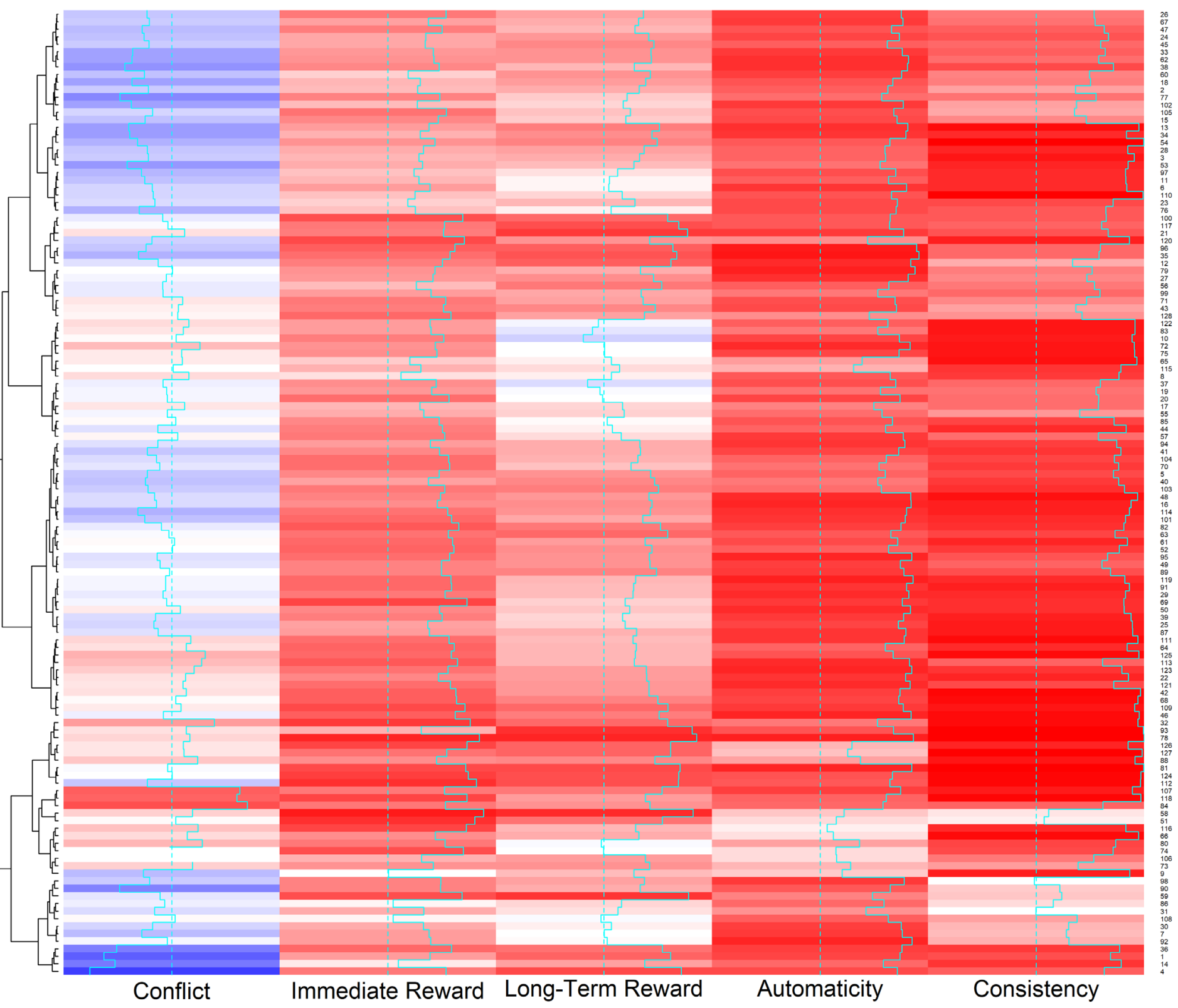


Figure SM-8. A heatmap that visualizes correlations between behavior regularity and individual factors from the Situated Action Cycle (Conflict, Immediate Reward, Long-Term Reward, Automaticity, and Consistency). The six correlations for each of the 115 participants in Study 3 appear in a single row. As a cell becomes increasingly red, the correlation was increasingly positive. As a cell becomes increasingly blue, the correlation was increasingly negative. As a cell becomes increasingly white, the correlation increasingly approached 0 . On the left, a hierarchical clustering dendrogram establishes groups of participants having similar prediction vectors (from hierarchical clustering with the Ward D measure). Table 4 in

the main text summarizes the correlations shown below.

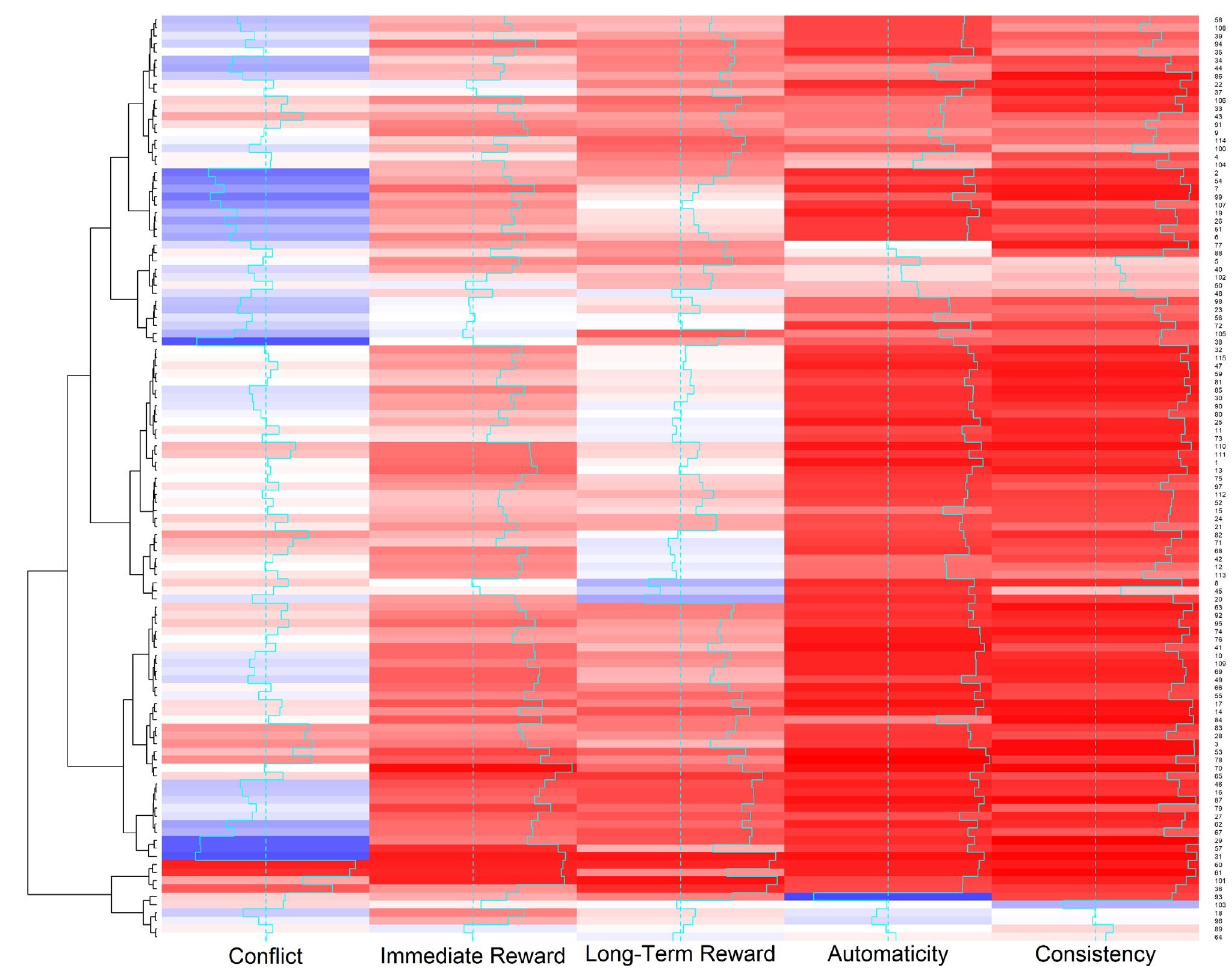




\section{Regression analyses that assessed self-control and neuroticism}

These next regression analyses produced the results that were displayed in Figure 7 and 8 of the main text.

To test the predicted valence $\times$ self-control interaction (Figure 7), we modeled behavior regularity with valence, self-control, and their interaction as predictors. Analogously, to test the predicted valence $\times$ neuroticism interaction, we modeled behavior regularity with valence, neuroticism, and their interaction as predictors (Figure 8). Both analyses used the analysis pipeline in Section 4 of the SM to establish Model 1, Model 2, and Model 3 for Studies 1-all, 2, and 3.

The results are shown in the rows of Table SM-7 labelled "V $\times \mathrm{S}$ (partial)" and "V $\times \mathrm{N}$ (partial)"). Estimates of the standardized regression coefficients in Model 2 and $\Delta R^{2}$ in Model 3 provide two measures of effect size. As can be seen, each predicted interaction survived maximal testing in Model 2 for all three studies, explaining $5 \%$ to $6 \%$ unique variance in behavior regularity. 
Table SM-7. Results for the valence $\mathrm{x}$ self-control interactions ( $\mathrm{V} \times \mathrm{S})$ and valence $\mathrm{x}$ neuroticism interactions (V x $\mathrm{N})$ from group regressions in Studies 1-all, 2, and 3. The partial models predicted behavior regularity with only valence and self-control or neuroticism, whereas the full models further added consistency, immediate reward, long-term reward, conflict, and automaticity (in Study 2, social approval was included as well). See the text for further details. Section 4 of the SM describes the analysis pipeline used to produce Models 1, 2, and 3.

DV: regularity

\begin{tabular}{|c|c|c|c|c|c|c|c|c|c|c|c|}
\hline \multirow[b]{2}{*}{ Study } & \multirow[b]{2}{*}{ Predictors } & & & \\
\hline & & Estimate & SE & $t$ & Estimate & SE & $t$ & $R^{2}$ & AIC & $\Delta R^{2}$ & $\triangle \mathrm{AIC}$ \\
\hline \multirow[t]{2}{*}{ 1-all } & $\mathrm{V} \times \mathrm{S}$ (partial) & .12 & .008 & 14.89 & .12 & .018 & 6.40 & 39 & 24758 & -5 & 495 \\
\hline & V x S (full) & .02 & .009 & 1.86 & & & & & & & \\
\hline \multirow[t]{2}{*}{2} & $\mathrm{~V} \times \mathrm{S}$ (partial) & .13 & .006 & 19.31 & .13 & .016 & 7.64 & 36 & 39088 & -5 & 803 \\
\hline & V x S (full) & .03 & .009 & 3.69 & .02 & .013 & 1.93 & 67 & 27467 & & \\
\hline \multirow[t]{2}{*}{3} & $\mathrm{~V} \times \mathrm{S}$ (partial) & .13 & .009 & 15.81 & .13 & .021 & 6.39 & 38 & 22554 & -6 & 553 \\
\hline & V x S (full) & .03 & .010 & 2.96 & .02 & .017 & 1.23 & 65 & 16379 & & \\
\hline \multirow[t]{2}{*}{ 1-all } & $\mathrm{V} \times \mathrm{N}$ (partial) & -.09 & .008 & -11.22 & -.09 & .019 & -4.69 & 39 & 24795 & -5 & 461 \\
\hline & V x N (full) & -.01 & .008 & -0.67 & & & & & & & \\
\hline \multirow[t]{2}{*}{2} & $\mathrm{~V} \times \mathrm{N}$ (partial) & -.09 & .007 & -13.88 & -.09 & .018 & -5.02 & 37 & 39096 & -5 & 799 \\
\hline & V x N (full) & -.00 & .010 & -0.41 & & & & & & & \\
\hline \multirow[t]{2}{*}{3} & $\mathrm{~V} \times \mathrm{N}$ (partial) & -.09 & .009 & -10.57 & -.09 & .023 & -3.96 & 38 & 22561 & -6 & 546 \\
\hline & V x N (full) & -.01 & .009 & -1.25 & & & & & & & \\
\hline
\end{tabular}

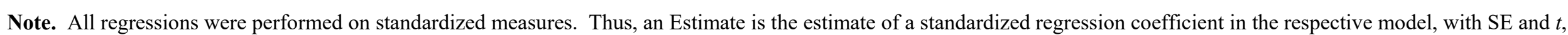
being the standard error and $t$ value of the estimate. $R^{2}$ is the total variance explained by Model 2 , and $\Delta R^{2}$ is the amount of variance explained by the main effect or interaction dropped in Model 3 (both in percentage points). AIC is the value of the Akaike Information Criterion for Model 2, and $\Delta$ AIC is its change for Model 3. 


\section{Stepwise regressions to establish processes from the Situated Action Cycle that underlie self-control and neuroticism}

These analyses explore the interactions in Figure 7 and 8 reported in the main text, producing the further related results also displayed there in Figures 9 and 10

To establish factors from the Situated Action Cycle that underlie self-control and neuroticism, we added the $\mathrm{SAM}^{2}$ predictors for consistency, immediate reward, long-term reward, conflict, and automaticity to the "partial" regression models in Table SM-7, thereby creating the "full" models shown there (see Section 9 of the SM). If the interactions just reported in Figures 7 and 8 of the main text disappear, then the $\mathrm{SAM}^{2}$ predictors explain the variance associated with self-control and neuroticism. If, however, these interactions remain, then these predictors do not explain variance associated with the personality measures.

Table SM-7 in the previous section presents the results of these regressions in the rows labelled "V $\times \mathrm{S}$ (full)" and "V $\times \mathrm{N}$ (full)"). As can be seen, both interactions disappeared when all five $\mathrm{SAM}^{2}$ predictors were added to the regression models, indicating that these predictors explained the variance in self-control and neuroticism (Figure SM-9 and SM-10 plot these interactions from the full models).

We next used stepwise regression to identify the specific $\mathrm{SAM}^{2}$ mechanisms that explained variance in these interactions. Specifically, each step added the mechanisms, one at a time, into a partial Model 2 and assessed how much doing so decreased the estimated regression coefficient for the interaction of interest. After each step, we added the remaining mechanism that decreased the coefficient the most. Every Model 2 contained random intercepts for behaviors and participants, random slopes for valence, self-control and valence $\times$ self-control, no random slopes for the added $\mathrm{SAM}^{2}$ predictors, and all two-way and three-way interactions. Section 4 of the SM describes the regression procedure in detail

Figure 9 in the main text presents results from the stepwise process for the valence $\times$ self-control interaction. Across studies, as self-control increased, automaticity, consistency, long-term reward, and immediate reward explained the increased regularity of positive behaviors and the decreased regularity of negative behaviors. Figure 10 in the main text presents the analogous results for the valence $\times$ neuroticism interaction. Across studies, as neuroticism increased, automaticity, long-term reward, and consistency explained the decreased performance of positive behaviors and the increased performance of negative behaviors.

Tables SM-8 and SM-9 here present the statistical details for both stepwise analyses. Specifically, Table SM-8 documents the regression results at each step of the stepwise process when evaluating the valence $\times$ self-control interaction, as does Table SM-9 for the valence $\times$ neuroticism interaction. See the table headings and the main article for further details. 
Figure SM-9. The absence of the interaction of behavior valence with self-control across Studies 1-all, 2 , and 3 . Each interaction modeled here was established in a mixed-effect regression analysis that predicted behavior regularity as a function of valence and self-control, along with the SAM ${ }^{2}$ predictors for consistency, immediate reward, long-term reward, conflict, and automaticity (with all variables standardized prior to analysis). Valence was an a priori variable that contrasted positive versus negative behaviors (Table 1). See the full models in Table SM-7 for analysis details.
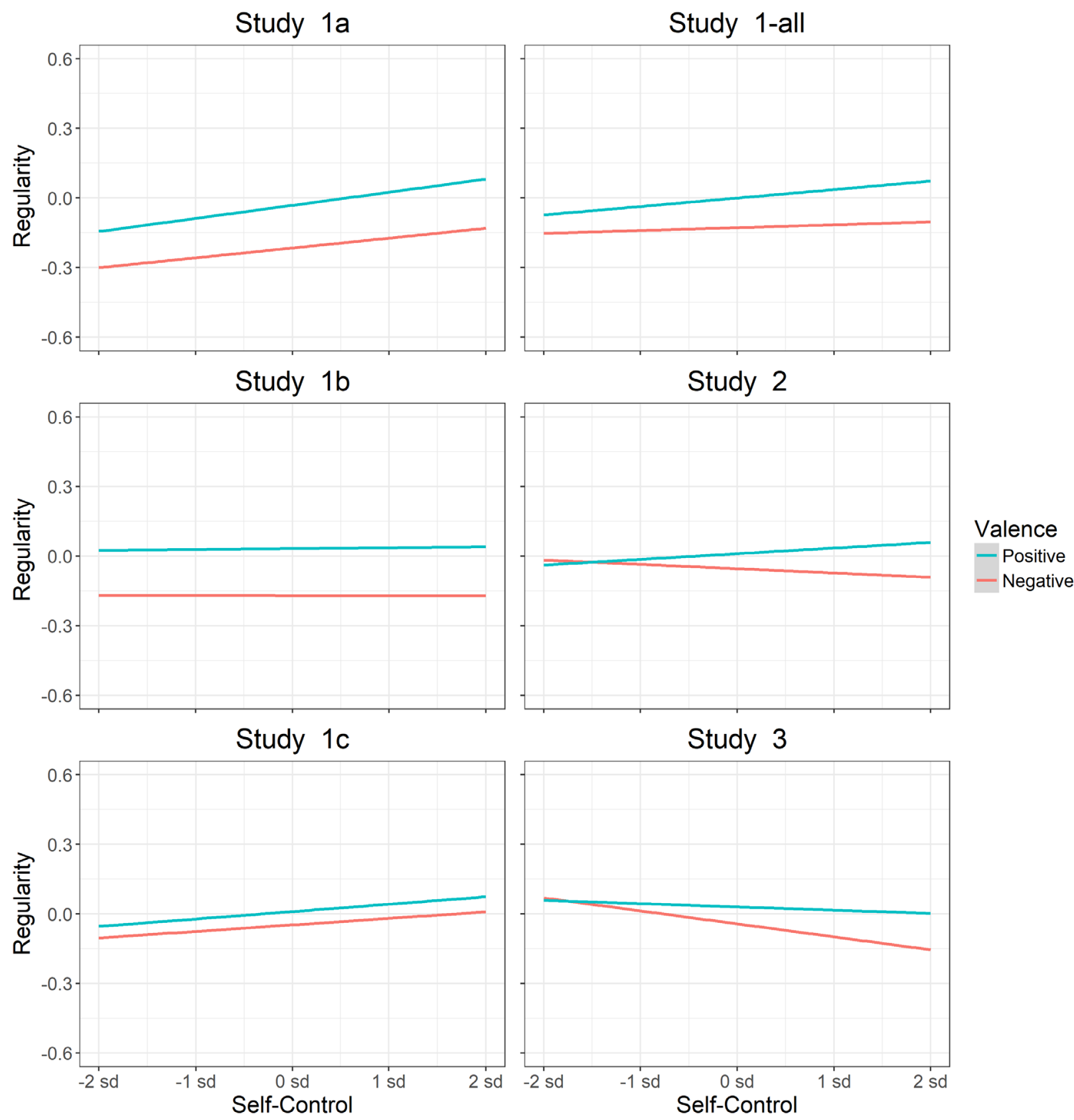
Figure SM-10. The absence of the interaction of behavior valence with neuroticism across Studies 1 -all, 2 , and 3 . Each interaction modeled here was established in a mixed-effect regression analysis that predicted behavior regularity as a function of valence and neuroticism, along with the SAM ${ }^{2}$ predictors for consistency, immediate reward, long-term reward, conflict, and automaticity (with all variables standardized prior to analysis). Valence was an a priori variable that contrasted positive versus negative behaviors (Table 1). See the full models in Table SM-7 for analysis details.

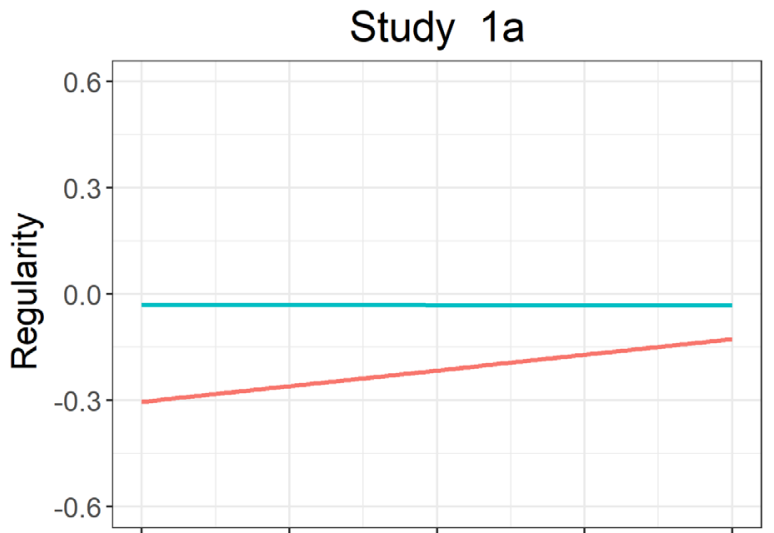

Study $1 b$

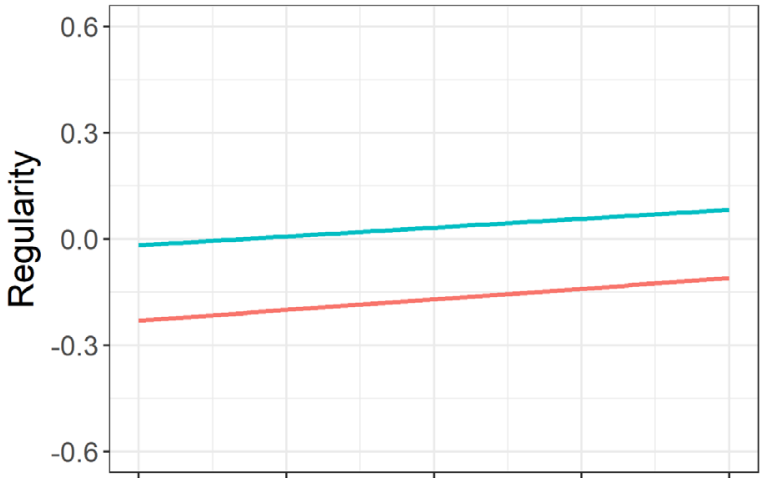

Study 1c

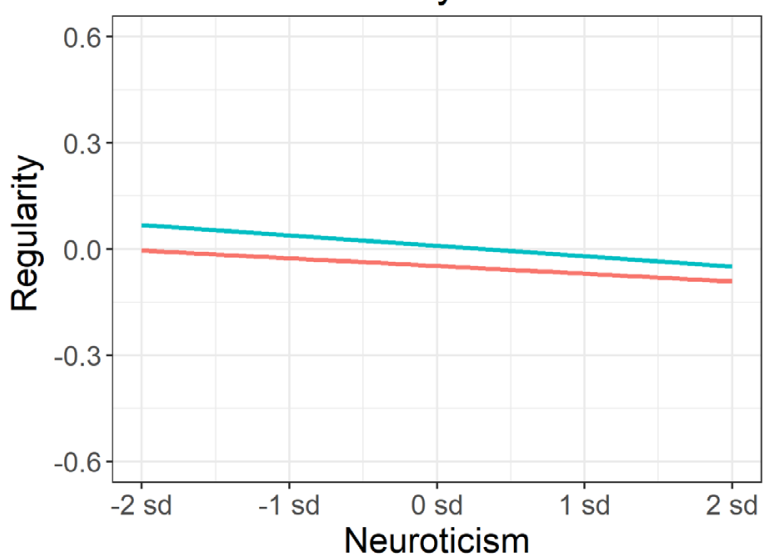

Study 1-all

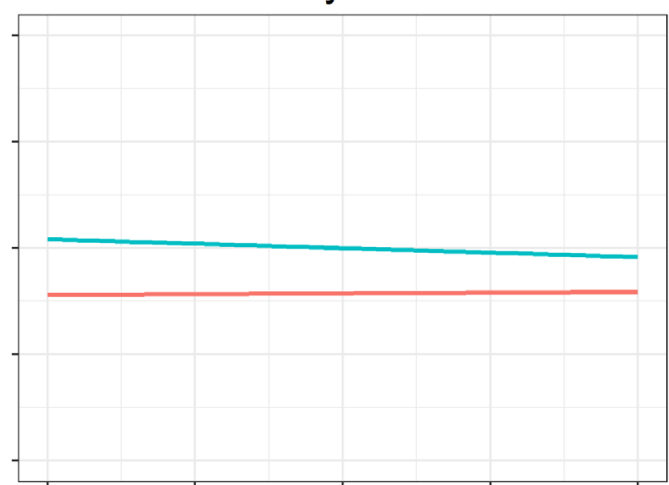

Study 2

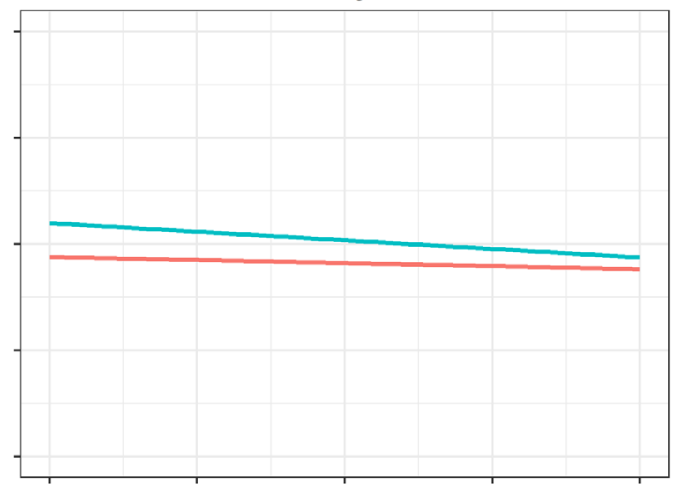

Study 3

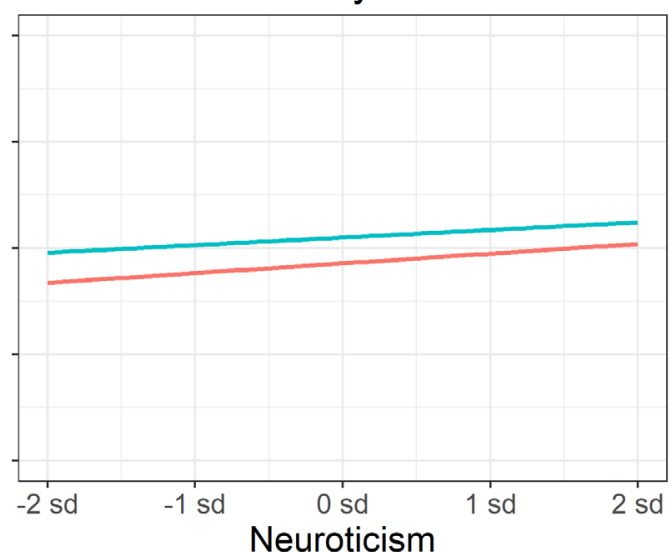

\section{Valence}

- Positive

- Negative 
Table SM-8. Results from stepwise regression to establish the $\mathrm{SAM}^{2}$ predictors that explained variance in the valence $\times$ self-control interaction in Studies 1-all, 2, and 3 (Figure 7). In the original analyses (the first row for each study), behavior regularity was regressed onto only valence and self-control in Model 2 from the analysis pipeline in section 4 of the SM to test the valence $\times$ self-control interaction maximally. The five primary $\mathrm{SAM}^{2}$ predictors (Figure 1) were then added one at a time into the original regression to assess how much each alone decreased the coefficient for the valence $\times$ self-control interaction $\left(e^{2} \mathrm{~V}_{\mathrm{V}} \mathrm{S}\right)$. The predictor that decreased est $\mathrm{V} \times \mathrm{S}$ the most is shown in the next row for the study, along with test statistics for the est $V_{\times} \mathrm{S}$ interaction and the overall Model 2. In four further iterations of the stepwise process, the remaining $\mathrm{SAM}^{2}$ predictors were again added one by one to identify the predictor that next decreased the est $\mathrm{V}_{\times} \mathrm{S}$ interaction the most. These results are shown in the third, fourth, fifth, and sixth rows for each study. Figure 9 in the main text plots each sequence of interactions that resulted from the stepwise analysis. Each model contained random intercepts for participants and behaviors, random slopes for valence, self-control and valence $\times$ self-control, no random slopes for the added $\mathrm{SAM}^{2}$ predictors, and all two-way and three-way interactions.

\begin{tabular}{|c|c|c|c|c|c|c|}
\hline \multicolumn{2}{|c|}{ DV: regularity } & \multicolumn{3}{|c|}{$\mathrm{V} \times \mathrm{S}$ interaction } & \multicolumn{2}{|c|}{ Model 2} \\
\hline Study & Predictors & Estimate & SE & $t$ & $\overline{\mathbf{R}^{2}}$ & AIC \\
\hline \multirow[t]{6}{*}{ 1-all } & Original & .12 & .018 & 6.42 & 39 & 24763 \\
\hline & + Automaticity & .05 & .013 & 3.72 & 52 & 20962 \\
\hline & + Immediate & .03 & .014 & 1.98 & 57 & 20118 \\
\hline & + Consistency & .02 & .011 & 1.66 & 69 & 17000 \\
\hline & + Long-term & .01 & .013 & 1.18 & 69 & 16868 \\
\hline & + Conflict & .02 & .013 & 1.25 & 70 & 16789 \\
\hline \multirow[t]{6}{*}{2} & Original & .13 & .016 & 7.64 & 36 & 39088 \\
\hline & + Consistency & .06 & .012 & 5.45 & 56 & 31995 \\
\hline & + Automaticity & .04 & .009 & 4.13 & 64 & 28812 \\
\hline & + Long-term & .03 & .010 & 2.92 & 65 & 28286 \\
\hline & + Immediate & .02 & .010 & 2.38 & 67 & 27682 \\
\hline & + Conflict & .03 & .010 & 2.77 & 67 & 27509 \\
\hline \multirow[t]{6}{*}{3} & Original & .14 & .022 & 6.46 & 38 & 22553 \\
\hline & + Automaticity & .06 & .017 & 3.45 & 54 & 18727 \\
\hline & + Long-term & .03 & .019 & 1.39 & 55 & 18415 \\
\hline & + Immediate & .02 & .019 & 0.92 & 58 & 18083 \\
\hline & + Conflict & .01 & .020 & 0.57 & 59 & 18003 \\
\hline & + Consistency & .02 & .016 & 1.01 & 65 & 16393 \\
\hline
\end{tabular}

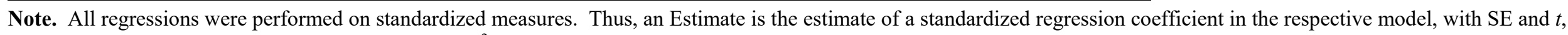
being the standard error and $t$ value of the estimate. $\mathrm{R}^{2}$ is the total variance explained (in percentage points), and AIC is the value of the Akaike Information Criterion. 
Table SM-9. Results from stepwise regression to establish the $\mathrm{SAM}^{2}$ predictors that explained variance in the valence $\times$ neuroticism interaction in Studies 1-all, 2, and 3 (Figure 7). In the original analyses (the first row for each study), behavior regularity was regressed onto only valence and neuroticism in Model 2 from the analysis pipeline in section 4 of the SM to test the valence $\times$ neuroticism interaction maximally. The five primary $\mathrm{SAM}^{2}$ predictors (Figure 1) were then added one at a time into the original regression to assess how much each alone decreased the coefficient for the valence $\times$ neuroticism interaction $\left(\right.$ est $\left._{\mathrm{V} \times \mathrm{N}}\right)$. The predictor that decreased est $\mathrm{V}_{\times \mathrm{N}}$ the most is shown in the next row for the study, along with test statistics for the est $V_{\times N}$ interaction and the overall Model 2. In four further iterations of the stepwise process, the remaining $\mathrm{SAM}^{2}$ predictors were again added one by one to identify the predictor that next decreased the est $V_{\times N}$ interaction the most. These results are shown in the third, fourth, fifth, and sixth rows for each study. Figure 9 in the main text plots each sequence of interactions that resulted from the stepwise analysis. Each model contained random intercepts for participants and behaviors, random slopes for valence, neuroticism and valence $\times$ neuroticism, no random slopes for the added $\mathrm{SAM}^{2}$ predictors, and all two-way and three-way interactions.

\begin{tabular}{|c|c|c|c|c|c|c|}
\hline \multicolumn{2}{|c|}{ DV: regularity } & \multicolumn{3}{|c|}{$\mathrm{V} \times \mathrm{N}$ interaction } & \multicolumn{2}{|c|}{ Model 2} \\
\hline Study & Predictors & $\overline{\text { Estimate }}$ & $\overline{\mathbf{S E}}$ & $t$ & $\overline{\mathbf{R}^{2}}$ & $\overline{\mathrm{AIC}}$ \\
\hline \multirow[t]{6}{*}{ 1-all } & Original & -.09 & .019 & -4.70 & 39 & 24795 \\
\hline & + Long-term & -.05 & .020 & -2.70 & 41 & 24208 \\
\hline & + Consistency & -.02 & .014 & -1.26 & 60 & 19660 \\
\hline & + Automaticity & -.01 & .012 & -1.07 & 68 & 17369 \\
\hline & + Immediate & -.01 & .011 & -1.16 & 69 & 16823 \\
\hline & + Conflict & -.01 & .012 & -0.95 & 70 & 16758 \\
\hline \multirow[t]{6}{*}{2} & Original & -.09 & .018 & -5.02 & 37 & 39096 \\
\hline & + Automaticity & -.05 & .012 & -4.08 & 48 & 34159 \\
\hline & + Consistency & -.03 & .009 & -3.24 & 63 & 28946 \\
\hline & + Long-term & -.02 & .010 & -1.76 & 65 & 28390 \\
\hline & + Immediate & -.02 & .010 & -1.71 & 66 & 27755 \\
\hline & + Conflict & -.02 & .010 & -1.68 & 67 & 27626 \\
\hline \multirow[t]{6}{*}{3} & Original & -.09 & .024 & -3.71 & 38 & 22589 \\
\hline & + Automaticity & -.02 & .017 & -1.24 & 53 & 18756 \\
\hline & + Long-term & .00 & .018 & 0.20 & 55 & 18457 \\
\hline & + Consistency & -.01 & .015 & -0.81 & 63 & 16720 \\
\hline & + Immediate & -.01 & .015 & -0.56 & 64 & 16501 \\
\hline & + Conflict & -.01 & .015 & -0.46 & 65 & 16438 \\
\hline
\end{tabular}

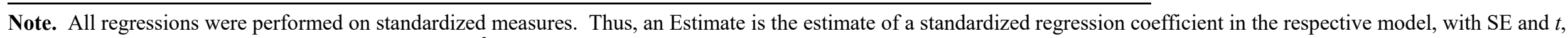
being the standard error and $t$ value of the estimate. $\mathrm{R}^{2}$ is the total variance explained (in percentage points), and AIC is the value of the Akaike Information Criterion. 


\section{Valence $\times$ personality interactions using rated valence in Study 2}

In the main text, the valence $\times$ self-control interaction in Figure 7 and the valence $\times$ neuroticism interaction in Figure 8 were both obtained originally using the a priori assignments of valence Table 1. Of interest here is whether these interactions also occur when rated valence replaces a priori valence.

To assess this issue, we modeled behavior regularity with rated valence, self-control, and their interaction as predictors. Analogously, to test the predicted valence $\times$ neuroticism interaction, we modeled behavior regularity with rated valence, neuroticism, and their interaction.

Figure SM-11 displays the valence $\times$ self-control interaction and the valence $\times$ neuroticism interaction for rated valence in Study 2. The analogous interactions with a priori valence from Study 2 are shown again here for the purpose of comparison (i.e., all interactions involving a priori valence were already presented in the main article).

As can be seen, the same interactions occur regardless of whether valence is assigned a priori or rated empirically. See the figure caption and the main article for further details. 


\section{Analysis of social approval as a predictor of behavior regularity}

Participants in Study 2 rated social approval for each behavior in Table 1. For each behavior participants were asked to judge, "How good or bad do people in general view this behaviour?", using a scale from -5 to 5 , with the labels: Very bad, Somewhat bad, Neutral, Somewhat good, Very good. The intraclass correlation for inter-rater agreement was .58 (ICC2).

Of interest is whether including social approval as a predictor improved the prediction of behavior regularity. To assess this issue in Study 2, we replaced a priori valence with rated valence and added social approval to the models in Table SM-5 that previously assessed the prediction of behavior regularity. Both rated valence and social approval were included to assess the roles of these closely related predictors together. Whereas rated valence assessed how individuals view positive versus negative behaviors themselves, social approval assessed how individuals believe that others view positive versus negative behaviors. Of interest was the relative contributions of these two evaluative judgments to explaining behavior regularity.

Table SM-10 presents the results for the main effects from Models 1, 2, and 3. The top panel of Figure SM-12 presents the estimated regression coefficients from Model 2. Importantly, the overall patterns were highly similar to those in Table SM-5 in the SM and Figure 4 in the main text. Again, consistency and then automaticity were the best predictors of behavior regularity, followed by immediate and then long-term reward, with conflict being unrelated. Interestingly, rated valence was a somewhat stronger predictor of behavior regularity than was a priori valence, suggesting that individuals judged behaviors more positively as they performed them more regularly.

To our surprise, social approval was negatively related to behavior regularity. This result is surprising, given that the correlation between social approval and regularity was positive $(r=.24)$. We subsequently discovered that social approval became negative in the regression because of its high collinearity with rated valence $(r=.76)$. When rated valence was removed from the regression model, social approval became a positive predictor, indicating that its original negative coefficient resulted from high collinearity. These results indicate that rated valence largely captured the importance of social approval. Social approval does not need to be included if valence is in the predictive model. 

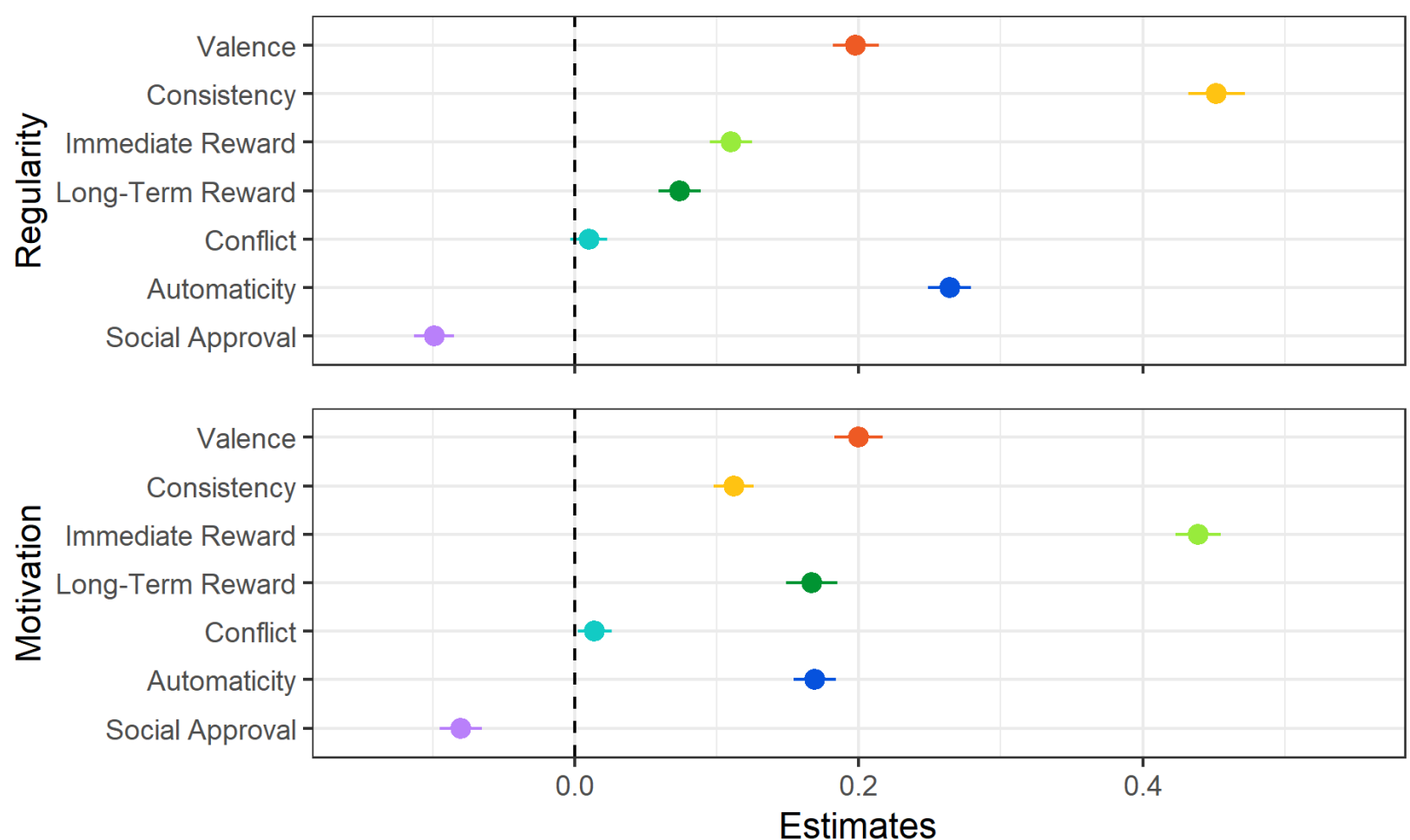

Figure 12. Estimated regression coefficients for the prediction of behavior regularity (top) and behavior motivation (bottom) in Study 2, including predictors for rated valence, consistency, immediate reward, long-term reward, conflict, automaticity, and social approval. All coefficients are standardized and were established in Model 2 of the analysis pipeline (see section 4 of the SM for details). The line for each coefficient is its standard error. Tables SM-10 and SM-11 provides details of the relevant regression analyses. 
Table SM-10. Complete results from the group regressions in Study 2 for the prediction of behavior regularity, including predictors for rated valence, consistency, immediate reward, long-term reward, conflict, automaticity, social approval, self-control, and neuroticism. Results for all main effects are shown, along with two- and three-way interactions in Model 1 where $t>|1.96|$. Section 4 of the SM describes the analysis pipeline used to produce Models 1, 2, and 3.

\begin{tabular}{|c|c|c|c|c|c|c|c|c|c|c|}
\hline \multirow{2}{*}{$\begin{array}{l}\text { DV: regularity } \\
\text { Predictor }\end{array}$} & \multicolumn{3}{|c|}{ Model 1} & \multicolumn{5}{|c|}{ Model 2} & \multicolumn{2}{|c|}{ Model 3} \\
\hline & Estimate & SE & $t$ & Estimate & SE & $t$ & $R^{2}$ & AIC & $\Delta R^{2}$ & AIC \\
\hline Consistency (Cs) & .45 & .008 & 54.28 & .45 & .020 & 23.01 & 69 & 26347 & -10 & 29910 \\
\hline Immediate Reward (I) & .11 & .009 & 11.87 & .11 & .015 & 7.41 & 68 & 27054 & -1 & 27362 \\
\hline Long-term Reward (L) & .07 & .010 & 7.29 & .07 & .016 & 4.73 & 68 & 27002 & -2 & 27275 \\
\hline Automaticity (A) & .27 & .008 & 32.15 & .26 & .015 & 17.44 & 68 & 26959 & -3 & 28221 \\
\hline Social Approval (So) & -.10 & .010 & -1.17 & -.10 & .014 & -7.04 & 68 & 27093 & -1 & 27325 \\
\hline Self-control (S) & .00 & .016 & 0.21 & & & & & & & \\
\hline Neuroticism $(\mathrm{N})$ & -.01 & .016 & -0.65 & & & & & & & \\
\hline $\mathrm{V} \times \mathrm{Cf}$ & -.05 & .009 & -5.19 & -.05 & .010 & -4.35 & 69 & 26926 & -2 & 27249 \\
\hline Cs x A & .04 & .006 & 7.30 & .01 & .010 & 0.45 & 72 & 25269 & & \\
\hline Cs $x$ So & -.02 & .009 & -2.27 & -.02 & .011 & -1.75 & 71 & 26149 & & \\
\hline Cs $x \mathrm{~S}$ & .03 & .006 & 5.07 & .03 & .015 & 2.30 & 70 & 26340 & -3 & 27248 \\
\hline I x L & -.02 & .007 & -2.28 & -.02 & .010 & -2.23 & 69 & 26888 & -2 & 27227 \\
\hline Ix A & -.03 & .006 & -4.16 & -.03 & .009 & -3.16 & 69 & 26707 & -2 & 27239 \\
\hline I x N & -.02 & .007 & -2.70 & -.02 & .010 & -2.00 & 68 & 27064 & -1 & 27229 \\
\hline $\mathrm{L} \times \mathrm{Cf}$ & -.02 & .007 & -2.61 & -.01 & .009 & -1.52 & 69 & 26902 & & \\
\hline $\mathrm{L} \times \mathrm{A}$ & -.03 & .008 & -3.65 & -.02 & .011 & -1.88 & 70 & 26669 & & \\
\hline $\mathrm{L} \times \mathrm{S}$ & .02 & .008 & 1.97 & .01 & .012 & 1.15 & 69 & 26993 & & \\
\hline $\mathrm{Cf} \times \mathrm{S}$ & .02 & .006 & 2.64 & .01 & .009 & 1.41 & 68 & 27104 & & \\
\hline
\end{tabular}




\begin{tabular}{|c|c|c|c|c|c|c|c|c|c|c|}
\hline $\mathrm{Cf} \times \mathrm{N}$ & .01 & .006 & 2.02 & .01 & .010 & 1.40 & 68 & 27115 & & \\
\hline A x So & .03 & .009 & 3.78 & .03 & .010 & 3.31 & 69 & 26814 & -2 & 27236 \\
\hline$A \times S$ & -.03 & .006 & -5.15 & -.03 & .011 & -3.00 & 68 & 26956 & -1 & 27248 \\
\hline So x S & -.02 & .009 & -2.48 & -.02 & .011 & -1.54 & 68 & 27087 & & \\
\hline$V \times C s \times A$ & -.05 & .007 & -6.65 & -.03 & .007 & -4.29 & 74 & 25328 & -8 & 27266 \\
\hline$V \times C s \times N$ & .03 & .009 & 3.54 & .02 & .010 & 1.67 & 72 & 26007 & & \\
\hline V x Cf x So & -.01 & .006 & -2.08 & -.01 & .007 & -1.37 & 69 & 26904 & & \\
\hline $\mathrm{V} \times \mathrm{Cf} \times \mathrm{S}$ & .02 & .009 & 2.60 & .02 & .010 & 2.27 & 69 & 26926 & -2 & 27229 \\
\hline $\mathrm{V} \times \mathrm{A} \times \mathrm{N}$ & -.02 & .009 & -2.57 & -.03 & .011 & -2.19 & 69 & 26780 & -2 & 27228 \\
\hline Cs X I x L & .04 & .006 & 6.21 & .02 & .007 & 2.66 & 73 & 25854 & -6 & 27260 \\
\hline Cs $x I \times A$ & -.02 & .005 & -3.82 & -.01 & .006 & -1.98 & 74 & 25433 & -7 & 27236 \\
\hline Cs $x$ Cf $x A$ & .02 & .005 & 4.19 & .01 & .006 & 1.37 & 73 & 25543 & & \\
\hline Cs $x$ Cf x S & -.03 & .005 & -4.78 & -.01 & .007 & -1.73 & 71 & 26175 & & \\
\hline Cs $x$ A x So & .04 & .007 & 5.32 & .03 & .007 & 3.83 & 74 & 25489 & -7 & 27250 \\
\hline I x L x A & -.03 & .006 & -4.17 & -.02 & .007 & -2.47 & 70 & 26668 & -3 & 27239 \\
\hline I x Cf x A & -.01 & .005 & -2.34 & -.01 & .006 & -1.42 & 70 & 26742 & & \\
\hline Cf $x$ A x So & .02 & .007 & 1.98 & .02 & .009 & 1.93 & 70 & 26727 & & \\
\hline
\end{tabular}

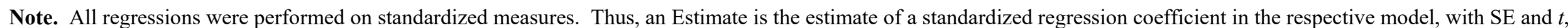
being the standard error and $t$ value of the estimate. $R^{2}$ is the total variance explained by Model 2 , and $\Delta R^{2}$ is the amount of variance explained by the main effect or interaction dropped in Model 3 (both in percentage points). AIC is the value of the Akaike Information Criterion for Models 2 and 3. 


\section{Predicting behavior motivation}

Participants in Study 2 rated social approval for each behavior in Table 1. For each behavior participants were asked to judge, "How strongly are you motivated to perform this behaviour in the relevant situations??", using a scale from -5 to 5, with the labels: Not at all, Somewhat, A lot. The intraclass correlation for inter-rater agreement was .22 (ICC2).

Of interest was establishing factors from the Situated Action Cycle for habitual behavior in Figure 1 that predict behavior motivation and comparing them to the factors that predict behavior regularity. Because motivation often results from anticipated reward, we predicted that immediate and long-term reward would be more important for predicting behavior motivation than for predicting behavior regularity. Additionally, because of the explicit focus on behavior motivation, we predicted that longterm reward would become especially important, exceeding the importance of immediate reward.

To test these predictions in Study 2, we predicted behavior motivation instead of behavior regularity with the same model that included rated valence and social approval in Table SM-10 (top). Table SM13 and the bottom half of Figure SM-12 present the results.

As predicted, both immediate and long-term reward increased in importance for predicting behavior motivation, relative to predicting behavior regularity. Unexpectedly, however, immediate reward was the most important predictor of motivation (not long-term reward), more than tripling in magnitude, relative to its magnitude for the prediction of regularity. Long-term reward doubled in importance but was about a third as important as immediate reward. As reward became more important for behavior motivation, consistency and automaticity become less important.

Interestingly, however, both remained important, suggesting that behavior strength produces impetus for behavior motivation. Again, the co-linearity of social approval and rated valence caused social approval to predict motivation negatively (as found in the previous section for social approval as a predictor of behavior regularity). When rated valence was removed, social approval became positively related to motivation. 
Table SM-11. Complete results from the group regressions in Study 2 for the prediction of behavior motivation, including predictors for rated valence, consistency, immediate reward, long-term reward, conflict, automaticity, social approval, self-control, and neuroticism. Results for all main effects are shown, along with two- and three-way interactions in Model 1 where $t>|1.96|$. Section 4 of the SM describes the analysis pipeline used to produce Models 1, 2, and 3.

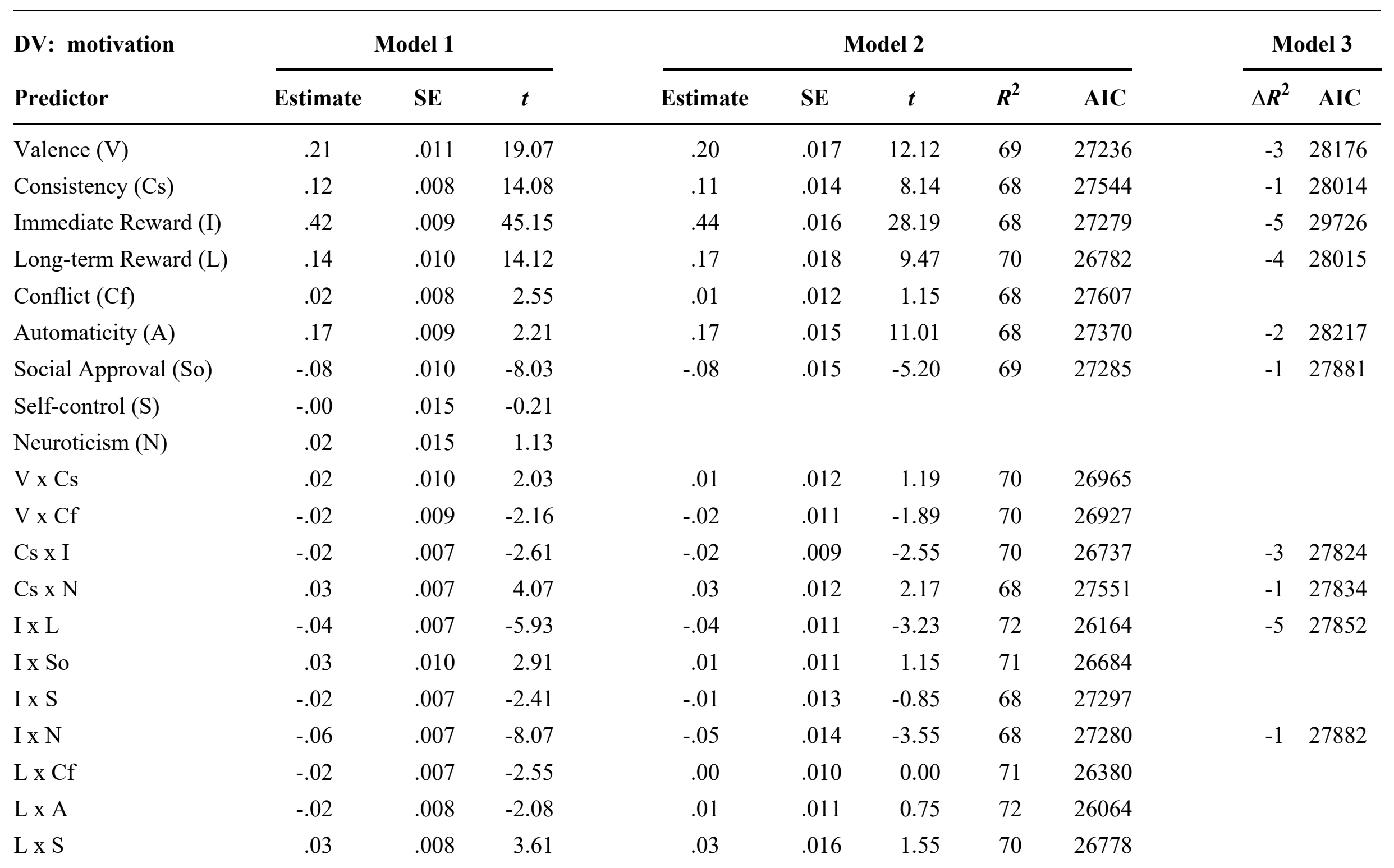




\begin{tabular}{|c|c|c|c|c|c|c|c|c|c|c|}
\hline $\mathrm{L} \times \mathrm{N}$ & -.04 & .008 & -4.43 & -.03 & .016 & -1.88 & 70 & 26785 & & \\
\hline Cf $x$ So & -.02 & .008 & -2.86 & -.01 & .011 & -1.22 & 70 & 27014 & & \\
\hline Cf $x \mathrm{~S}$ & -.02 & .006 & -2.73 & -.01 & .011 & -1.12 & 68 & 27601 & & \\
\hline $\mathrm{Cf} \times \mathrm{N}$ & -.03 & .006 & -4.40 & -.02 & .011 & -1.62 & 68 & 27617 & & \\
\hline$A \times S$ & -.03 & .006 & -3.92 & -.03 & .013 & -2.16 & 68 & 27381 & -1 & 27832 \\
\hline V x Cs x So & -.02 & .007 & -3.19 & -.02 & .008 & -2.29 & 70 & 26854 & -3 & 27827 \\
\hline V x I x L & -.02 & .008 & -2.86 & -.03 & .009 & -2.69 & 74 & 26015 & -6 & 27825 \\
\hline V x I x So & .02 & .007 & 3.45 & .01 & .008 & 0.57 & 72 & 26387 & & \\
\hline V x I x S & -.03 & .010 & -2.97 & -.02 & .011 & -2.22 & 72 & 26459 & -4 & 27826 \\
\hline V x I x N & -.04 & .010 & -3.77 & -.02 & .011 & -2.15 & 72 & 26457 & -4 & 27831 \\
\hline $\mathrm{V} \times \mathrm{L} \times \mathrm{A}$ & .02 & .009 & 2.81 & .03 & .010 & 2.78 & 73 & 25989 & -6 & 27825 \\
\hline V x L x So & .02 & .007 & 3.31 & .01 & .008 & 1.65 & 72 & 26571 & & \\
\hline V x Cf $x$ So & -.02 & .006 & -3.44 & -.01 & .008 & -1.65 & 71 & 26862 & & \\
\hline $\mathrm{V} \times \mathrm{Cf} \times \mathrm{N}$ & -.02 & .009 & -2.63 & -.02 & .012 & -1.57 & 70 & 26978 & & \\
\hline$V \times A \times S$ & .04 & .010 & 4.20 & .03 & .011 & 3.06 & 71 & 26705 & -4 & 27835 \\
\hline$V \times A \times N$ & .03 & .010 & 3.45 & .02 & .011 & 1.90 & 71 & 26709 & & \\
\hline $\mathrm{V} \times \mathrm{S} \times \mathrm{N}$ & .03 & .007 & 4.51 & .03 & .013 & 2.06 & 69 & 27248 & -2 & 27837 \\
\hline Cs $x \mathrm{I} \times \mathrm{L}$ & .02 & .006 & 3.14 & .00 & .007 & 0.12 & 74 & 25877 & & \\
\hline Cs $\times I \times A$ & -.01 & .005 & -2.53 & -.01 & .007 & -1.04 & 72 & 26355 & & \\
\hline Cs $x \mathrm{I} \times \mathrm{N}$ & -.03 & .006 & -4.35 & -.02 & .007 & -2.57 & 70 & 26889 & -3 & 27836 \\
\hline Cs $x$ L x A & -.02 & .007 & -3.19 & -.03 & .008 & -3.35 & 73 & 25942 & -6 & 27827 \\
\hline Cs $x$ L x So & .03 & .008 & 3.64 & .02 & .010 & 1.87 & 72 & 26281 & & \\
\hline Cs $x$ L x S & .02 & .008 & 2.37 & .02 & .009 & 2.07 & 71 & 26434 & -4 & 27823 \\
\hline Cs $x A \times N$ & .02 & .006 & 3.61 & .01 & .006 & 0.85 & 69 & 27227 & & \\
\hline I x Cf x So & .03 & .007 & 3.77 & .02 & .008 & 2.81 & 72 & 26397 & -5 & 27831 \\
\hline I x A x So & -.03 & .008 & -3.47 & -.02 & .008 & -2.13 & 73 & 26007 & -6 & 27829 \\
\hline $\mathrm{I} \times \mathrm{A} \times \mathrm{S}$ & -.01 & .006 & -2.32 & -.02 & .007 & -2.33 & 71 & 26586 & -4 & 27822 \\
\hline
\end{tabular}




\begin{tabular}{lrrrrrrrrrr} 
I x A x N & -.02 & .006 & -3.14 & -.02 & .007 & -2.63 & 71 & 26587 & -4 & 27827 \\
I x So x N & .02 & .009 & 1.96 & .03 & .010 & 2.52 & 71 & 26710 & -4 & 27821 \\
L x A x So & -.02 & .009 & -2.80 & -.03 & .010 & -3.07 & 73 & 26039 & -6 & 27825 \\
L x A x N & -.03 & .008 & -3.14 & -.02 & .009 & -2.05 & 72 & 26153 & -5 & 27827 \\
A x So x S & -.02 & .009 & -2.53 & -.02 & .010 & -2.42 & 70 & 26807 & -3 & 27823 \\
\hline
\end{tabular}

Note. All regressions were performed on standardized measures. Thus, an Estimate is the estimate of a standardized regression coefficient in the respective model, with SE and $t$, being the standard error and $t$ value of the estimate. $R^{2}$ is the total variance explained by Model 2, and $\Delta R^{2}$ is the amount of variance explained by the main effect or interaction dropped in Model 3 (both in percentage points). AIC is the value of the Akaike Information Criterion for Models 2 and 3. 


\section{Assessing explicit awareness of factors that predict behavior regularity}

Because habitual behaviors relatively implicit and automatic, participants should be relatively unaware of the specific SAM ${ }^{2}$ factors from the Situated Action Cycle in Figure 1 most associated with their individual patterns of behavior regularity. To assess this prediction in Study 3, participants produced 5 responses on a 0 to 100 scale to estimate how much overall influence each factor has on their performance of the 80 behaviors (where the factors were consistency, immediate reward, longterm reward, conflict, and automaticity). Figure SM-13 presents the items used to collect these responses.

For each participant, a second vector of 5 values was created that contained the actual correlations of these 5 factors with behavior regularity in their individual data. These two vectors were then correlated for each participant, indicating how well their prediction estimates correlated with their actual predictive relations.

Figure SM-14 presents the results, with each point being the correlation between the estimated and observed prediction vectors for one of the 115 participants in Study 3. As can be seen, the median correlation across participants was .25 , with a broad range from -.85 to +.98 , suggesting large individual differences. As we predicted, many participants did not appear to have much awareness of what actually predicted their behavior regularity. No other measure correlated well with these values, suggesting that they may not be systematic (e.g., self-control correlated -.18, neuroticism correlated -.19).

Interestingly, the average estimates across participants departed substantially from the overall relationships between the $\mathrm{SAM}^{2}$ predictors and behavior regularity actually observed in Figures 7 and 11. Specifically, participants' average estimates (on a 0 to 100 scale) were 63.33 for consistency, 75.45 for immediate reward, 63.01 for long-term reward, 59.29 for conflict, and 75.47 for automaticity. Participants mistakenly perceived immediate and long-term reward as being comparable in importance to consistency and automaticity, when consistency and automaticity were actually much more important. Participants mistakenly believed that conflict was quite important, when actually it was not. Participants mistakenly believed that automaticity was more important than consistency, when consistency was actually more important. These misconceptions indicate that, overall, participants had little awareness of the factors associated with behavior regularity. 
Figure SM-13. The screen in Study 3 used to collect each participant's 5 estimates of how much each of the $\mathrm{SAM}^{2}$ predictors affect how often they Please think about the various behaviours that you judged earlier. What factors influence how frequently you perform these behaviours?

Specifically, we're interested in knowing how much you think each of the factors below influences the frequency of the behaviours assessed in this study. For each factor, please move the slider to the point that best indicates how much influence it has on how frequently you perform these behaviours.

If you think that a factor has a very strong influence on how frequently you perform these behaviours, position the slider towards the 100 end of the scale.

If you think that a factor has little or no influence on how frequently you perform these behaviours, position the slider towards the 0 end of the scale.

If you think that a factor has moderate influence on how frequently you perform these behaviours, position the slider in the middle of the scale.

How much does each of the factors below influence how frequently you perform a behaviour?

How much does the automaticity of a behaviour (i.e., performing it with little thought or effort) influence how frequently you perform it?

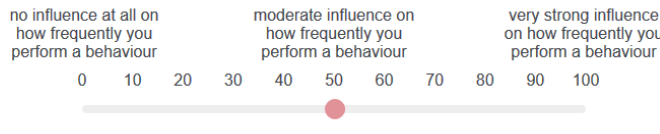

How much does the consistency of the situations where you perform a behaviour (i.e., the same place and time) influence how frequently you perform it?

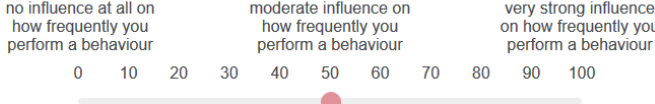

How much does being conflicted about a behaviour (i.e., both wanting and not-wanting to do it) influence how frequently you perform it?

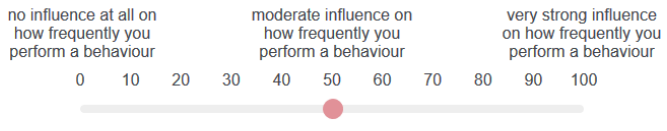

How much do the long-term consequences of a behaviour (i.e., how good it will be for you in the long-term) influence how frequently you perform it?

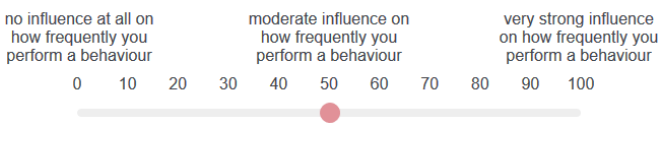

How much do the immediate consequences of a behaviour (i.e., how good it will make you feel in the moment) influence how frequently you perform it? 


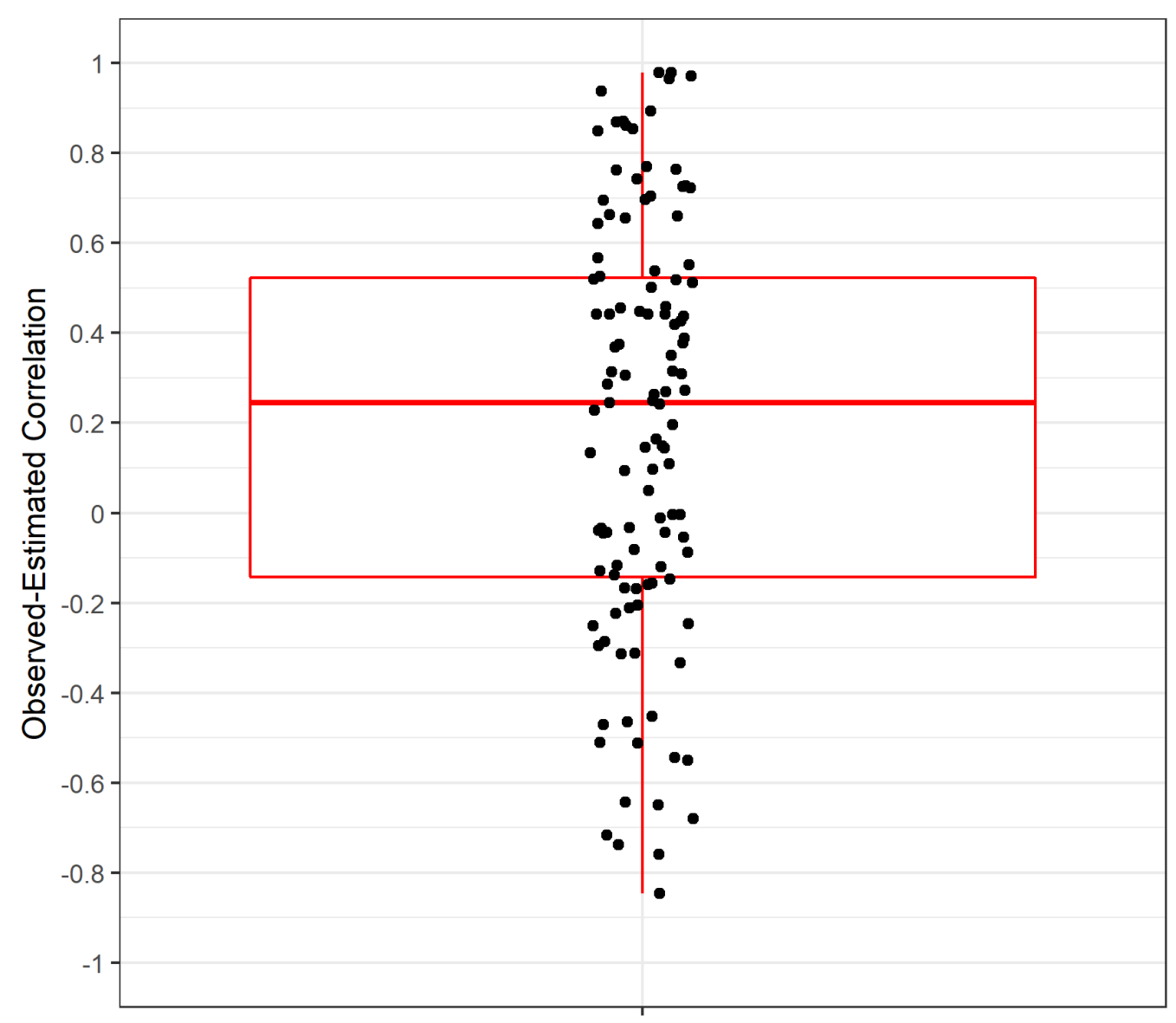

Participant

Figure SM-14. Individual correlations for participants between their estimated prediction vectors and their observed prediction vectors (Study 3 ). Specifically, each point represents the correlation

between: (1) a participant's estimates of how much overall influence each of the 6 predictors had on their regularity of performing the 80 behaviors, and (2) the 6 correlations of the 6 predictors with behavior regularity in their individual data. 